REPRESENTATION THEORY

An Electronic Journal of the American Mathematical Society

Volume 13, Pages 82-140 (April 8, 2009)

S 1088-4165(09)00348-3

\title{
CHARACTER SHEAVES ON DISCONNECTED GROUPS, $\mathrm{X}$
}

\author{
G. LUSZTIG
}

\begin{abstract}
We classify the unipotent character sheaves on a fixed connected component of a reductive algebraic group under a mild condition on the characteristic of the ground field.
\end{abstract}

\section{INTRODUCTION}

Throughout this paper, $G$ denotes a fixed, not necessarily connected, reductive algebraic group over an algebraically closed field $\mathbf{k}$ with a fixed connected component $D$ which generates $G$. This paper is part of a series [L9] which attempts to develop a theory of character sheaves on $D$.

Our main result here is the classification of "unipotent" character sheaves on $D$ (under a mild assumption on the characteristic of $\mathbf{k}$ ). This extends the results of L3. IV, V], which applied to the case where $G=G^{0}$. While in the case of $G=$ $G^{0}$ the classification of unipotent character sheaves is essentially the same as the classification of unipotent representations of a split connected reductive group over $\mathbf{F}_{q}$, the classification in the general case is essentially the same as the classification of unipotent representations of a not necessarily split connected reductive group over $\mathbf{F}_{q}$ given in [L14].

We now describe the content of the various sections in more detail. $\S 43$ contains some preparatory material concerning (extended) Hecke algebra and two-sided cells which are used later in the study of unipotent character sheaves. In $\S 44$ we study the unipotent character sheaves in connection with Weyl group representations and two-sided cells. (But it turns out that the method of associating a two-sided cell to a unipotent character sheaf along the lines of [L3, III] is better for our purposes than the one in $\S 41$.) A number of results in this section are conditional (they depend on a cleanness property and/or on a parity property); they will become unconditional in $\S 46$. In $\S 45$ we show that the problem of classifying the unipotent character sheaves on $D$ can be reduced to the analogous problem in the case where $G^{0}$ is simple and $G$ has trivial centre. In $\S 46$ we extend the results of [L3, IV, V], on the classification of unipotent character sheaves on $D$ from the case $G=G^{0}$ to the general case.

Erratum to [L9, V]; in line 4 of 25.1 replace "syatem" by "system".

Erratum to [L9, VI]: on p. 383 lines $-25,-24$ replace $Z$ by ${ }^{\prime} \bar{Z}^{\mathbf{s}}$ and $\Delta_{j}^{0}$ by $\Delta_{j}$.

Erratum to [L9, VII]: on p. 248 , line 4 of 35.5 replace $G^{0} F$ by $G^{0 F}$.

Received by the editors July 17, 2008.

2000 Mathematics Subject Classification. Primary 20G99.

Supported in part by the National Science Foundation.

(C)2009 American Mathematical Society Reverts to public domain 28 years from publication 
Erratum to [L9, VIII]: on p. 346, line 14 replace the first $k$ by $k^{\prime}$; on p. 350 , lines 3 and 4 of 39.6 delete "The restriction of", "to"; on p. 350, line 6 of 39.6 replace first $\sigma$ by $x$; the 5 lines preceding 39.8 ("If $n=3$ then $\ldots$ is proved") should be replaced by the following text:

"If $n=3$, then $W$ must be of type $D_{4}, \Gamma$ is the alternating group in the four letters $a, b, c, d, W^{(K)}$ is either $\{1\}$ or $\mathbf{Z} / 2$ (with trivial $\Gamma$-action) or the $\mathbf{Z} / 2$-vector space spanned by $a, b, c, d$ with the obvious $\Gamma$-action. It is enough to show that $E=E^{\prime} \otimes E^{\prime \prime}$ where $E^{\prime}$ is a $\overline{\mathfrak{U}}\left[W^{(K)} \Gamma\right]$-module defined over $\mathbf{Q}$ and $E^{\prime \prime}$ is a $\overline{\mathfrak{U}}\left[W^{(K)} \Gamma\right]$ module of dimension 1 over $\overline{\mathfrak{U}}$. If $W^{(K)} \Gamma$ has order $\leq 2$, this follows from the fact that:

(a) Any simple $\overline{\mathfrak{U}}[\Gamma]$-module is either defined over $\mathbf{Q}$ or has dimension 1 .

(Indeed, if it has dimension $>1$, then it is the restriction to $\Gamma$ of the 3 -dimensional reflection representation of the symmetric group in the four letters, which is defined over Q.)

Now assume that $W^{(K)}$ has order $>2$. We can find a homomorphism $\epsilon: W^{(K)} \rightarrow$ $\overline{\mathfrak{U}}^{*}$ (with image in $\{1,-1\}$ ) whose stabilizer in $\Gamma$ is denoted by $\Gamma_{\epsilon}$ and a simple $\overline{\mathfrak{U}}\left[\Gamma_{\epsilon}\right]$ module $E_{0}$ such that $E=\operatorname{Ind}_{W^{(K)} \Gamma_{\epsilon}}^{W_{\epsilon}^{(K)}}\left(E_{\epsilon} \otimes E_{0}\right)$; here $E_{\epsilon}$ is the one-dimensional $\overline{\mathfrak{U}}\left[W^{(K)}\right]$-module defined by $\epsilon$ (necessarily defined over $\mathbf{Q}$ ). If $\Gamma_{\epsilon}=\Gamma$, then $E=$ $E_{\epsilon} \otimes E_{0}$ where $E_{0}$ is as in (a) and the desired result follows. If $\Gamma_{\epsilon}$ has order 2, then $E_{0}$ is defined over $\mathbf{Q}$, hence $E$ is defined over $\mathbf{Q}$. If $\Gamma_{\epsilon} \neq \Gamma$ and $\Gamma_{\epsilon}$ is not of order 2, then $\Gamma_{\epsilon}$ is of order $3, E_{0}$ is the restriction to $\Gamma_{\epsilon}$ of a one-dimensional $\overline{\mathfrak{U}}[\Gamma]$-module $E^{\prime \prime}$ and we have $E=E^{\prime} \otimes E^{\prime \prime}$ where $E^{\prime}=\operatorname{Ind}_{W^{(K)} \Gamma_{\epsilon}}^{W^{(K)}}\left(E_{\epsilon} \otimes \overline{\mathfrak{U}}\right)$ is defined over Q. Hence the proposition holds in this case. The proposition is proved."

Erratum to [L9, IX]: on p. 354 , line -8 replace $V_{\lambda}, V_{\lambda}^{D}$ by $\Omega_{\lambda}, \Omega_{\lambda}^{D}$; on p. 354 , line -7 replace 34.4 by 34.2 ; on p. 355 , lines $-8,-13$ replace $V_{\lambda}$ by $\Omega_{\lambda}$; on p. 359 , first line of 40.8 replace $c_{y . \lambda}$ by $c_{y, \nu}$; on the preceding line replace $i n$ by $\in$; on p. 361, line 9 insert "," before $\mathcal{L}$; on p. 363, line 6 before "Let" insert: "Let $\dot{\mathcal{L}}_{w}^{\sharp}=I C\left(\bar{Z}_{\emptyset, D}^{w}, \dot{\mathcal{L}}_{w}\right)$."; on p. 365, second line of 41.4, two ) are missing; on p. 366, last displayed line of 41.4 replace $\dashv A$ by $\dashv \mathfrak{e}(A)$; on p. 368, line 2 remove "the condition that"; on p. 369 , line7 a ) is missing; on p. 371 , line 1 replace $H_{n}$ by $H$; on p. 372 , line 4 of 42.5 replace $\otimes \mathcal{A}$ by $\otimes_{\mathcal{A}}$; on p. 376 , line -22 replace $W W$ by $\mathbf{W}$; on p. 376 , line -10 replace $H_{n}^{D, \tilde{\mathcal{A}}} D$ by $H_{n}^{D, \tilde{\mathcal{A}}}$; on p. 377 , line -10 replace $v t$ by $\vartheta$; on p. 378 , line 6 replace $\Delta$ by $D$.

Notation. Let $\epsilon:=\epsilon_{D}$ be as in 26.2. If $X$ is an algebraic variety over $\mathbf{k}$ and $K \in \mathcal{D}(X)$, we write $H^{i}(K)$ instead of ${ }^{p} H^{i}(K)$. If $K \in \mathcal{D}(X)$, we set $g r_{1} K=$ $\sum_{i \in \mathbf{Z}}(-1)^{i} H^{i}(K)$, an element of the Grothendieck group of the category of perverse sheaves on $X$. The cardinal of a finite set $X$ is denoted by $|X|$.

\section{Contents}

43. Preparatory results on Hecke algebras.

44. Unipotent character sheaves and two-sided cells.

45. Reductions.

46. Classification of unipotent character sheaves. 


\section{Preparatory Results on Hecke algebras}

43.1. This section contains some preparatory material concerning (extended) Hecke algebras and two-sided cells which will be used later in the study of unipotent character sheaves.

We fix an even integer $c \geq 2$ which is divisible by $\left|G / G^{0}\right|$. Let $\Gamma$ be a cyclic group of order $c$ with generator $\varpi$. Let $\tilde{\mathbf{W}}$ be the semidirect product of $\mathbf{W}$ with $\Gamma$ (with $\mathbf{W}$ normal) where $\varpi x \varpi^{-1}=\epsilon(x)$ for $x \in \mathbf{W}$. Note that the group $\mathbf{W}^{D}$ in 34.2 is naturally a quotient of $\tilde{\mathbf{W}}$, via $x \varpi^{i} \mapsto x \underline{D}^{i}$ with $x \in \mathbf{W}, i \in \mathbf{Z}$. Let $\operatorname{Irr}(\mathbf{W})$ be the category whose objects are the simple (or equivalently, absolutely simple) $\mathbf{Q}[\mathbf{W}]$-modules. Let $\operatorname{Irr}^{\epsilon}(\mathbf{W})$ be the category whose objects are the simple $\mathbf{Q}[\mathbf{W}]$-modules $E_{0}$ such that $\operatorname{tr}\left(x, E_{0}\right)=\operatorname{tr}\left(\epsilon(x), E_{0}\right)$ for all $x \in \mathbf{W}$. Let $\operatorname{Mod}(\tilde{\mathbf{W}})$ be the category whose objects are the $\mathbf{Q}[\tilde{\mathbf{W}}]$-modules of finite dimension over $\mathbf{Q}$. Let $\operatorname{Irr}(\tilde{\mathbf{W}})$ be the subcategory of $\operatorname{Mod}(\tilde{\mathbf{W}})$ consisting of those objects that remain simple on restriction to $\mathbf{Q}[\mathbf{W}]$. Let $\underline{\operatorname{Irr}}(\tilde{\mathbf{W}})$ be a set of representatives for the isomorphism classes in $\operatorname{Irr}(\tilde{\mathbf{W}})$. Let $\iota$ be the object of $\operatorname{Irr}(\tilde{\mathbf{W}})$ whose underlying $\mathbf{Q}$-vector space is $\mathbf{Q}$ with $\mathbf{W}$ acting trivially and $\varpi$ acting as multiplication by -1 . Note that if $E \in \operatorname{Irr}(\tilde{\mathbf{W}})$, then $\left.E\right|_{\mathbf{Q}[\mathbf{W}]} \in \operatorname{Irr}^{\epsilon}(\mathbf{W})$. Conversely, we show:

(a) for any $E_{0} \in \operatorname{Irr}^{\epsilon}(\mathbf{W})$, the set $\left\{E \in \underline{\operatorname{Irr}}(\tilde{\mathbf{W}}) ;\left.E\right|_{\mathbf{Q}[\mathbf{W}]} \cong E_{0}\right\}$ has exactly two elements; one is isomorphic to the other tensored with $\iota$.

From [L14, 3.2] we see that there exists a linear map of finite order $\gamma: E_{0} \rightarrow E_{0}$ such that $\gamma(x(e))=\epsilon(x)(\gamma(x))$ for any $e \in E_{0}, x \in \mathbf{W}$. (We use the following property of $\epsilon$ : if $s, s^{\prime} \in \mathbf{I}$ are such that $s s^{\prime}$ has order $\geq 4$, then $s, s^{\prime}$ are in distinct orbits of $\epsilon$ on I.) Moreover, from the proof in [L14, 3.2] we see that $\gamma$ can be chosen so that $\gamma^{c^{\prime}}=1$ where $c^{\prime}$ is the order of the permutation $\epsilon: \mathbf{W} \rightarrow \mathbf{W}$. In particular, we have $\gamma^{c}=1$. This proves that the set in (a) is nonempty. The remainder of (a) is immediate.

Let $\mathfrak{E}$ be a subset of $\underline{\operatorname{Irr}}(\tilde{\mathbf{W}})$ such that $\left\{\left.E\right|_{\mathbf{Q}[\mathbf{W}]} ; E \in \mathfrak{E}\right\}$ represents each isomorphism class in $\operatorname{Irr}^{\epsilon}(\mathbf{W})$ exactly once.

43.2. Recall the notation $\mathcal{A}=\mathbf{Z}\left[v, v^{-1}\right]$. Define $l: \tilde{\mathbf{W}} \rightarrow \mathbf{N}$ by $l\left(x \varpi^{i}\right)=l(x)$ for $x \in \mathbf{W}, i \in \mathbf{Z}$; here $l: \mathbf{W} \rightarrow \mathbf{N}$ is the standard length function. Let $w_{0}$ be the longest element of $\mathbf{W}$. Let $\tilde{H}$ be the $\mathcal{A}$-algebra with 1 with generators $\tilde{T}_{w}(w \in \tilde{\mathbf{W}})$ and relations

$$
\begin{gathered}
\tilde{T}_{w} \tilde{T}_{w^{\prime}}=\tilde{T}_{w w^{\prime}} \text { for } w, w^{\prime} \in \tilde{\mathbf{W}} \text { with } l\left(w w^{\prime}\right)=l(w)+l\left(w^{\prime}\right), \\
\tilde{T}_{s}^{2}=\tilde{T}_{1}+\left(v-v^{-1}\right) \tilde{T}_{s} \text { for } s \in \mathbf{I} .
\end{gathered}
$$

We have a surjective $\mathcal{A}$-algebra homomorphism $\zeta: \tilde{H} \rightarrow H_{1}^{D}, \tilde{T}_{x \varpi^{i}} \mapsto \tilde{T}_{x \underline{D}^{i}}$ for $x \in \mathbf{W}, i \in \mathbf{Z}$ where $H_{1}^{D}$ is the algebra $H_{n}^{D}$ in 34.4 (with $n=1$ ); thus, a number of properties of $\tilde{H}$ can be deduced from the corresponding properties of $H_{1}^{D}$ in $\S 34$.

Let $\xi \mapsto \xi^{\dagger}$ be the $\mathcal{A}$-algebra isomorphism $\tilde{H} \rightarrow \tilde{H}$ such that $\tilde{T}_{w}^{\dagger}=(-1)^{l(w)} \tilde{T}_{w^{-1}}^{-1}$ for all $w \in \tilde{\mathbf{W}}$. Let ${ }^{-}: \mathcal{A} \rightarrow \mathcal{A}$ be the ring isomorphism such that $\overline{v^{i}}=v^{-i}$ for $i \in \mathbf{Z}$. Let $^{-}: \tilde{H} \rightarrow \tilde{H}, \xi \mapsto \bar{\xi}$ be the ring isomorphism such that $\overline{a \tilde{T}_{w}}=\bar{a} \tilde{T}_{w^{-1}}^{-1}$ for $w \in \tilde{\mathbf{W}}$, 
$a \in \mathcal{A}$; this isomorphism commutes with $\xi \mapsto \xi^{\dagger}$. For $w \in \tilde{\mathbf{W}}$ we set

$$
\begin{gathered}
c_{w}=\sum_{y \in \mathbf{W} ; y \leq x} v^{l(y)-l(x)} P_{y, x}\left(v^{2}\right) \tilde{T}_{y \varpi^{i}} \in \tilde{H}, \\
\tilde{c}_{w}=\sum_{z \in \mathbf{W} ; x \leq z}(-1)^{l(z)-l(x)} v^{l(x)-l(z)} P_{w_{0} z, w_{0} x}\left(v^{2}\right) \tilde{T}_{z \varpi^{i}} \in \tilde{H},
\end{gathered}
$$

where $w=x \varpi^{i}(x \in \mathbf{W}, i \in \mathbf{Z})$ and

$$
P_{y, x}(\mathbf{q})=\sum_{j \in \mathbf{Z}} n_{y, x, j} \mathbf{q}^{j / 2}, \quad n_{y, x, j} \in \mathbf{Z}
$$

are the polynomials defined in [KL1] for the Coxeter group W. Note that $n_{y, x, j}=0$ unless $j \in 2 \mathbf{Z}$ and $n_{x, x, j}=\delta_{j, 0}$. We have $c_{w}=c_{x} \tilde{T}_{\varpi^{i}}$ and $\overline{c_{w}}=c_{w}$. It follows that

$$
c_{w}^{\dagger}=\sum_{y \in \mathbf{W} ; y \leq x}(-1)^{l(y)} v^{-l(y)+l(x)} P_{y, x}\left(v^{-2}\right) \tilde{T}_{y \varpi^{i}} \in \tilde{H}
$$

and $\overline{c_{w}^{\dagger}}=c_{w}^{\dagger}$.

Let $\tilde{H}^{v}=\mathbf{Q}(v) \otimes_{\mathcal{A}} \tilde{H}$, a $\mathbf{Q}(v)$-algebra. Let $\tilde{H}^{1}=\mathbf{Q} \otimes_{\mathcal{A}} \tilde{H}$ where $\mathbf{Q}$ is regarded as an $\mathcal{A}$-algebra under $v \mapsto 1$. We have $\tilde{H}^{1}=\mathbf{Q}[\tilde{\mathbf{W}}]$ (with $\tilde{T}_{w} \in \tilde{H}^{1}$ identified with $w \in \mathbf{Q}[\tilde{\mathbf{W}}]$ for $w \in \tilde{\mathbf{W}})$. Let $\left.\xi \mapsto \xi\right|_{v=1}$ be the ring homomorphism $\tilde{H} \rightarrow \tilde{H}^{1}$ given by $v \mapsto 1, \tilde{T}_{w} \mapsto w$ for $w \in \tilde{\mathbf{W}}$.

Let $H, H^{v}, H^{1}$ be the algebras defined like $\tilde{H}, \tilde{H}^{v}, \tilde{H}^{1}$ by replacing $\tilde{\mathbf{W}}$ by $\mathbf{W}$. We identify $H, H^{v}, H^{1}$ with subalgebras with 1 of $\tilde{H}, \tilde{H}^{v}, \tilde{H}^{1}$ in an obvious way. We have $H^{1}=\mathbf{Q}[\mathbf{W}]$. Note that $H$ is the same as the algebra $H_{n}$ in 31.2 (with $n=1$ ).

For $x, y \in \mathbf{W}$ we have $c_{x} c_{y}=\sum_{z \in \mathbf{W}} r_{x, y}^{z} c_{z}$ with $r_{x, y}^{z} \in \mathcal{A}$. There is a well-defined function $\mathbf{a}: \mathbf{W} \rightarrow \mathbf{N}$ such that for any $x, y, z \in \mathbf{W}$ we have $r_{x, y}^{z} \in v^{\mathbf{a}(z)} \mathbf{Z}\left[v^{-1}\right]$ and for any $z \in \mathbf{W}$ we have $r_{x, y}^{z} \notin v^{\mathbf{a}(z)-1} \mathbf{Z}\left[v^{-1}\right]$ for some $x, y \in \mathbf{W}$. For any $x, y, z \in \mathbf{W}$ we define $\gamma_{x, y, z^{-1}} \in \mathbf{Z}$ by $r_{x, y}^{z}=\gamma_{x, y, z^{-1}} v^{\mathbf{a}(z)} \bmod v^{\mathbf{a}(z)-1} \mathbf{Z}\left[v^{-1}\right]$.

We define a preorder $\preceq$ on $\mathbf{W}$ as follows: we say that $x^{\prime} \preceq x$ if there exists $x_{1}, x_{2}$ in $\mathbf{W}$ such that in the expansion (in $H$ ) $c_{x_{1}} c_{x} c_{x_{2}}=\sum_{y^{\prime} \in \mathbf{W}} r_{y^{\prime}} c_{y^{\prime}}$ with $r_{y^{\prime}} \in \mathcal{A}$, then we have $r_{x^{\prime}} \neq 0$. Let $\sim$ be the equivalence relation on $\mathbf{W}$ attached to $\preceq$. The equivalence classes for $\sim$ are called the two-sided cells of W. (See also KL1].) We write $x \prec y$ instead of $x \preceq y, x \nsim y$. It is known that $\mathbf{a}: \mathbf{W} \rightarrow \mathbf{N}$ is constant on each two-sided cell. If $\mathbf{c}, \mathbf{c}^{\prime}$ are two-sided cells, we write $\mathbf{c} \preceq \mathbf{c}^{\prime}$ instead of $x \preceq x^{\prime}$ for some/any $x \in \mathbf{c}, x^{\prime} \in \mathbf{c}^{\prime}$. This is a partial order on the set of two-sided cells; we also write $\mathbf{c} \prec \mathbf{c}^{\prime}$ instead of $\mathbf{c} \preceq \mathbf{c}^{\prime}, \mathbf{c} \neq \mathbf{c}^{\prime}$.

The free abelian group $H^{\infty}$ with basis $\left\{t_{x} ; x \in \mathbf{W}\right\}$ is regarded as a ring with multiplication given by $t_{x} t_{y}=\sum_{z \in \mathbf{W}} \gamma_{x, y, z^{-1}} t_{z}$ for $x, y \in \mathbf{W}$. This ring has a unit element of the form $\sum_{\delta \in \mathcal{D}} t_{\delta}$ where $\mathcal{D}$ is a well-defined subset of $\mathbf{W}$. We have $H^{\infty}=\bigoplus_{\mathbf{c}} H_{\mathbf{c}}^{\infty}$ (as rings) where $\mathbf{c}$ runs over the two-sided cells and $H_{\mathbf{c}}^{\infty}$ is the subgroup of $H^{\infty}$ generated by $\left\{t_{x} ; x \in \mathbf{c}\right\}$. Let $\tilde{H}^{\infty}$ be the free abelian group with basis $\left\{t_{x} \varpi^{i} ; x \in \mathbf{W}, i \in[0, c-1]\right\}$. We have naturally $H^{\infty} \subset \tilde{H}^{\infty}\left(t_{x}=t_{x} v^{0}\right)$. The group ring $\mathbf{Z}[\Gamma]$ is also naturally contained in $\tilde{H}^{\infty}$ by $\varpi^{i} \mapsto \sum_{d \in \mathcal{D}} t_{d} v^{i}$. We regard $\tilde{H}^{\infty}$ as a ring with 1 so that $H^{\infty}$ and $\mathbf{Z}[\Gamma]$ are subrings with 1 and $\varpi t_{x} \varpi^{-1}=t_{\varpi(x)}$ for $x \in \mathbf{W}$. We have a surjective ring homomorphism $\zeta^{\infty}: \tilde{H}^{\infty} \rightarrow H_{1}^{D, \infty}, t_{x} \varpi^{i} \mapsto$ $t_{x \underline{D}^{i}}$ for $x \in \mathbf{W}, i \in \mathbf{Z}$ where $H_{1}^{D, \infty}$ is the ring $H_{n}^{D, \infty}$ (with $n=1$ ) in 34.12. 
Define $\mathcal{A}$-linear maps $\Phi: H \rightarrow \mathcal{A} \otimes H^{\infty}, \tilde{\Phi}: \tilde{H} \rightarrow \mathcal{A} \otimes \tilde{H}^{\infty}$ by $\Phi\left(c_{x}^{\dagger}\right)=$ $\sum_{z \in \mathbf{W}, d \in \mathcal{D}, \mathbf{a}(d)=\mathbf{a}(z)} r_{x, d}^{z} t_{z}$ for $x \in \mathbf{W}, \tilde{\Phi}\left(c_{x \varpi^{i}}^{\dagger}\right)=\Phi\left(c_{x}^{\dagger}\right) \varpi^{i}$ for $x \in \mathbf{W}, i \in \mathbf{Z}$. Now $\Phi, \tilde{\Phi}$ are homomorphisms of rings with 1 . We have the commutative diagram

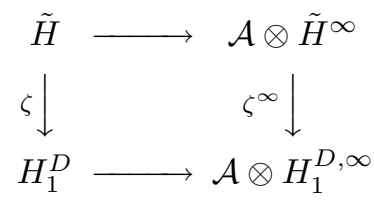

where the upper horizontal map is the composition of ${ }^{\dagger}: \tilde{H} \rightarrow \tilde{H}$ with $\tilde{\Phi}$ and the lower horizontal map is the map denoted by $\Phi$ in 34.1, 34.12 (which is not the same as the present $\Phi)$.

For any field $k$ let $H_{k}^{\infty}=k \otimes H^{\infty}, \tilde{H}_{k}^{\infty}=k \otimes \tilde{H}^{\infty}$. Let $\Phi^{v}: H^{v} \rightarrow H_{\mathbf{Q}(v)}^{\infty}$, $\tilde{\Phi}^{v}: \tilde{H}^{v} \rightarrow \tilde{H}_{\mathbf{Q}(v)}^{\infty}$ be the $\mathbf{Q}(v)$-algebra homomorphisms obtained from $\Phi, \tilde{\Phi}$ by extension of scalars. Let $\Phi^{1}: H^{1} \rightarrow H_{\mathbf{Q}}^{\infty}, \tilde{\Phi}^{1}: \tilde{H}^{1} \rightarrow \tilde{H}_{\mathbf{Q}}^{\infty}$ be the Q-algebra homomorphisms obtained from $\Phi, \tilde{\Phi}$ by extension of scalars. Now $\Phi^{v}, \tilde{\Phi}, \Phi^{1}, \tilde{\Phi}^{1}$ are algebra isomorphisms. Since the $\mathbf{Q}$-algebra $\mathbf{Q}[\mathbf{W}]=H^{1}$ is split semisimple, the same holds for the $\mathbf{Q}$-algebra $H_{\mathbf{Q}}^{\infty}$.

Now $\xi \mapsto \xi^{\dagger}$ induces by extension of scalars a $\mathbf{Q}(v)$-algebra isomorphism $\tilde{H}^{v} \rightarrow$ $\tilde{H}^{v}$ and a Q-algebra isomorphism $\tilde{H}^{1} \rightarrow \tilde{H}^{1}$; these leave $H^{v}, H^{1}$ stable and are denoted again by $\xi \mapsto \xi^{\dagger}$.

43.3. Let $E_{0} \in \operatorname{Irr}(\mathbf{W})$. We can view $E_{0}$ as a simple $H_{\mathbf{Q}}^{\infty}$-module $E_{0}^{\infty}$ via $\Phi^{1}$. Now $\mathbf{Q}(v) \otimes_{\mathbf{Q}} E_{0}^{\infty}$ is naturally a simple $H_{\mathbf{Q}(v)}^{\infty}$-module and this can be viewed as a simple $H^{v}$-module $E_{0}^{v}$ via $\Phi^{v}$.

Let $E \in \operatorname{Irr}(\tilde{\mathbf{W}})$. We can view $E$ as a simple $\tilde{H}_{\mathbf{Q}}^{\infty}$-module $E^{\infty}$ via $\tilde{\Phi}^{1}$. Now $\mathbf{Q}(v) \otimes_{\mathbf{Q}} E^{\infty}$ is naturally a $\tilde{H}_{\mathbf{Q}(v)}^{\infty}$-module and this can be viewed as an $\tilde{H}^{v}$-module $E^{v}$ via $\tilde{\Phi}^{v}$. By restriction, $E$ can be viewed as a simple $\mathbf{Q}[\mathbf{W}]=H^{1}$-module $E_{0}$. From the definitions we see that $E_{0}^{v}$ is the restriction of the $\tilde{H}^{v}$-module $E^{v}$ to $H^{v}$.

Let $E^{\prime}$ be the $\mathbf{Q}[\tilde{\mathbf{W}}]$-module with the same underlying $\mathbf{Q}[\mathbf{W}]$-module structure as $E$ but with the action of $\varpi$ equal to -1 times the action of $\varpi$ on $E$. Then $E^{\prime v}$ is defined. Clearly, $E^{\prime v}, E^{v}$ have the same underlying $H^{v}$-module and the action of $\tilde{T}_{\varpi}$ on $E^{\prime v}$ is equal to -1 times the action of $\tilde{T}_{\varpi}$ on $E^{v}$.

Let sgn be the object of $\operatorname{Irr}(\tilde{\mathbf{W}})$ with underlying vector space $\mathbf{Q}$ on which $w \in \tilde{\mathbf{W}}$ acts as multiplication by $(-1)^{l(w)}$. We set $E^{\dagger}=E \otimes \operatorname{sgn} \in \operatorname{Irr}(\tilde{\mathbf{W}})$.

43.4. Let $E \in \operatorname{Irr}(\tilde{\mathbf{W}})$. From the definitions, for any $\xi \in \tilde{H}, \zeta \in \tilde{H}^{\infty}$ we have:

$$
\operatorname{tr}\left(\xi, E^{v}\right) \in \mathcal{A},\left.\quad \operatorname{tr}\left(\xi, E^{v}\right)\right|_{v=1}=\operatorname{tr}\left(\left.\xi\right|_{v=1}, E\right), \quad \operatorname{tr}\left(\zeta, E^{\infty}\right) \in \mathbf{Z} .
$$

Hence it makes sense to write

$$
\operatorname{tr}\left(\xi, E^{v}\right)=\sum_{i \in \mathbf{Z}} \operatorname{tr}\left(\xi, E^{v} ; i\right) v^{i} \text { where } \operatorname{tr}\left(\xi, E^{v} ; i\right) \in \mathbf{Z} .
$$

More generally, for $\xi \in \tilde{H}^{v}$ we write $\operatorname{tr}\left(\xi, E^{v}\right)=\sum_{i \in \mathbf{Z}} \operatorname{tr}\left(\xi, E^{v} ; i\right) v^{i}$ (possibly infinite sum) where $\operatorname{tr}\left(\xi, E^{v} ; i\right) \in \mathbf{Q}$ (here $\operatorname{tr}\left(\xi, E^{v}\right) \in \mathbf{Q}(v)$ is viewed as a power series in $\mathbf{Q}((v)))$.

For any $\xi \in \tilde{H}$ we show:

$$
\operatorname{tr}\left(\xi,\left(E^{\dagger}\right)^{v}\right)=\operatorname{tr}\left(\xi^{\dagger}, E^{v}\right) .
$$


Let $E^{v \dagger}$ be the $\tilde{H}^{v}$-module whose underlying $\mathbf{Q}(v)$-module is $E^{v}$ but with $\xi \in \tilde{H}^{v}$ acting as $\xi^{\dagger}$ in the $\tilde{H}^{v}$-module $E^{v}$. Note that the $\tilde{H}^{v}$-module $E^{v \dagger}$ is simple and its restriction to an $H^{v}$-module is simple. Also, the assignment $E^{\prime} \mapsto E^{\prime v}$ defines a bijection between the set of isomorphism classes of objects of $\operatorname{Irr}(\tilde{\mathbf{W}})$ and the set of isomorphism classes of simple $\tilde{H}^{v}$-modules whose restriction to $H^{v}$ is simple. Thus we have $E^{v \dagger} \cong E_{1}^{v}$ for some $E_{1} \in \operatorname{Irr}(\tilde{\mathbf{W}})$. It is enough to show that $\left(E^{\dagger}\right)^{v} \cong E^{v \dagger}$ or that $\left(E^{\dagger}\right)^{v} \cong E_{1}^{v}$ as $\tilde{H}^{v}$-modules. Using (a) for $\xi \in \tilde{H}$ we have:

$$
\begin{aligned}
\operatorname{tr}\left(\xi_{v=1}, E_{1}\right) & =\operatorname{tr}\left(\xi, E_{1}^{v}\right)_{v=1}=\operatorname{tr}\left(\xi, E^{v^{\dagger}}\right)_{v=1}=\operatorname{tr}\left(\xi^{\dagger}, E^{v}\right)_{v=1} \\
& =\operatorname{tr}\left(\left.\xi^{\dagger}\right|_{v=1}, E\right)=\operatorname{tr}\left(\left.\xi\right|_{v=1}, E \otimes \operatorname{sgn}\right) .
\end{aligned}
$$

Thus, $\operatorname{tr}\left(w, E_{1}\right)=\operatorname{tr}\left(w, E^{\dagger}\right)$ for any $w \in \tilde{\mathbf{W}}$ so that $E_{1} \cong E^{\dagger}$ in $\operatorname{Irr}(\tilde{\mathbf{W}})$ and $\left(E^{\dagger}\right)^{v} \cong E_{1}^{v}$, as required.

For any $w \in \tilde{\mathbf{W}}$ we have:

$$
\operatorname{tr}\left(\tilde{T}_{w}^{-1}, E^{v}\right)=\operatorname{tr}\left(\tilde{T}_{w}, E^{v}\right) .
$$

The proof is the same as that of 34.17 (we use also (a)).

For any $\xi \in \tilde{H}$ we show:

$$
\operatorname{tr}\left(\bar{\xi}, E^{v}\right)=\overline{\operatorname{tr}\left(\xi, E^{v}\right)}
$$

We may assume that $\xi=c_{x \varpi^{j}}^{\dagger}$ with $x \in \mathbf{W}, j \in \mathbf{Z}$. Since $\bar{\xi}=\xi$, it is enough to verify:

$$
\sum_{z \in \mathbf{W}, d \in \mathcal{D}, \mathbf{a}(d)=\mathbf{a}(z)} r_{x, d}^{z} \operatorname{tr}\left(t_{z} \varpi^{j}, E^{\infty}\right)=\sum_{z \in \mathbf{W}, d \in \mathcal{D}, \mathbf{a}(d)=\mathbf{a}(z)} \overline{r_{x, d}^{z}} \operatorname{tr}\left(t_{z} \varpi^{j}, E^{\infty}\right) .
$$

This follows from the obvious identity $r_{x, y}^{z}=\overline{r_{x, y}^{z}}$ for any $x, y, z \in \mathbf{W}$.

For any $w \in \tilde{\mathbf{W}}$ we show:

$$
\operatorname{tr}\left(\tilde{T}_{w},\left(E^{\dagger}\right)^{v}\right)=(-1)^{l(w)} \overline{\operatorname{tr}\left(\tilde{T}_{w}, E^{v}\right)} .
$$

Using (b),(d), we see that the left-hand side of (e) equals

$$
\left.(-1)^{l(w)} \operatorname{tr}\left(\tilde{T}_{w^{-1}}^{-1}\right), E^{v}\right)=(-1)^{l(w)} \operatorname{tr}\left(\overline{\tilde{T}_{w}}, E^{v}\right)=(-1)^{l(w)} \overline{\operatorname{tr}\left(\tilde{T}_{w}, E^{v}\right)} .
$$

This proves (e).

43.5. For $E \in \operatorname{Irr}(\tilde{\mathbf{W}})$ we define $f_{E}^{v} \in \mathbf{Q}\left[v, v^{-1}\right], f_{E}^{\infty} \in \mathbf{Q}$ by

$$
\sum_{x \in \mathbf{W}} \operatorname{tr}\left(\tilde{T}_{x}, E^{v}\right)^{2}=f_{E}^{v} \operatorname{dim} E, \sum_{x \in \mathbf{W}} \operatorname{tr}\left(t_{x}, E^{\infty}\right)^{2}=f_{E}^{\infty} \operatorname{dim} E
$$

Note that $f_{E}^{v}, f_{E}^{\infty}$ depend only on $\left.E\right|_{\mathbf{Q}[\mathbf{W}]}$. Now $f_{E}^{v}$ is $\neq 0$; it specializes to $|\mathbf{W}| / \operatorname{dim} E$ for $v=1$. Since $E_{0}^{\infty}$ is a simple $H_{\mathbf{Q}}^{\infty}$-module, the integer $\operatorname{tr}\left(t_{x}, E_{0}^{\infty}\right)$ is $\neq 0$ for some $x \in \mathbf{W}$. Hence $f_{E}^{\infty} \neq 0$. For $E, E^{\prime}$ in $\operatorname{Irr}(\tilde{\mathbf{W}})$, the following holds:

(b) $\sum_{x \in \mathbf{W}} \operatorname{tr}\left(\tilde{T}_{x \varpi}, E^{v}\right) \operatorname{tr}\left(\tilde{T}_{x \varpi}, E^{\prime v}\right)$ equals $f_{E}^{v} \operatorname{dim} E$ if $E, E^{\prime}$ are isomorphic and equals 0 if $\left.E\right|_{\mathbf{Q}[\mathbf{W}]} \neq\left. E^{\prime}\right|_{\mathbf{Q}[W]}$.

This can be deduced from 34.15(c) using the commutative diagram in 43.2 (we use also 43.4(a)). Similarly,

(c) $\sum_{x \in \mathbf{W}} \operatorname{tr}(x \varpi, E) \operatorname{tr}\left(x \varpi, E^{\prime}\right)$ equals $|\mathbf{W}|$ if $E, E^{\prime}$ are isomorphic and equals 0 if $\left.\left.E\right|_{\mathbf{Q}[\mathbf{W}]} \not E^{\prime}\right|_{\mathbf{Q}[W]}$. 
43.6. Let $E_{0} \in \operatorname{Irr}(\mathbf{W})$. Let $E_{0}^{\infty}$ be the irreducible $H_{\mathbf{Q}}^{\infty}$-module corresponding to $E_{0}$ as in 43.3. Since $H_{\mathbf{Q}}^{\infty}=\bigoplus_{\mathbf{c}} \mathbf{Q} \otimes H_{\mathbf{c}}^{\infty}$ as $\mathbf{Q}$-algebras, there is a unique two-sided cell $\mathbf{c}=\mathbf{c}_{E_{0}}$ such that $E_{0}^{\infty}$ restricts to a simple module of the summand $\mathbf{Q} \otimes H_{\mathbf{c}}^{\infty}$ (and all other summands act as 0 on $E_{0}^{\infty}$ ). Let $a_{E_{0}}$ be the value of $\mathbf{a}$ on $\mathbf{c}_{E_{0}}$.

Let $E \in \operatorname{Irr}(\tilde{\mathbf{W}})$. We set $\mathbf{c}_{E}=\mathbf{c}_{E_{0}}, a_{E}=a_{E_{0}}$ where $E_{0}=\left.E\right|_{\mathbf{Q}[\mathbf{W}]} \in \operatorname{Irr}(\mathbf{W})$. We show:

(a) If $x \in \mathbf{W}$, then $\operatorname{tr}\left(c_{x \varpi}^{\dagger}, E^{v}\right)=\operatorname{tr}\left(t_{x} \varpi, E^{\infty}\right) v^{-a_{E}} \bmod v^{-a_{E}+1} \mathbf{Z}[v]$; equivalently, $\operatorname{tr}\left(c_{x \varpi}^{\dagger}, E^{v} ;-a_{E}\right)=\operatorname{tr}\left(t_{x} \varpi, E^{\infty}\right)$ and $\operatorname{tr}\left(c_{x \varpi}^{\dagger}, E^{v} ; \tilde{a}\right)=0$ for all $\tilde{a}<-a_{E}$.

(b) If $x \in \mathbf{W}$ and the action of $c_{x \varpi}^{\dagger}$ on $E^{v}$ is $\neq 0$, then $z \preceq x$ for some $z \in \mathbf{c}_{E}$. From the definition,

$$
\operatorname{tr}\left(c_{x \varpi}^{\dagger}, E^{v}\right)=\sum_{z \in \mathbf{W}, d \in \mathcal{D}, \mathbf{a}(d)=\mathbf{a}(z)} r_{x, d}^{z} \operatorname{tr}\left(t_{z} \varpi, E^{\infty}\right) .
$$

In the last sum we have $\operatorname{tr}\left(t_{z} \varpi, E^{\infty}\right)=0$ unless $z \in \mathbf{c}_{E}$ in which case $\mathbf{a}(z)=a_{E}$. For such $z$ we have $r_{x, d}^{z}=\gamma_{x, d, z^{-1}} v^{a_{E}} \bmod v^{a_{E}-1} \mathbf{Z}\left[v^{-1}\right]$, hence $r_{x, d}^{z}=\overline{r_{x, d}^{z}}=$ $\gamma_{x, d, z^{-1}} v^{-a_{E}} \bmod v^{-a_{E}+1} \mathbf{Z}[v]$ and

$$
\operatorname{tr}\left(c_{x \varpi}^{\dagger}, E^{v}\right)=\sum_{z \in \mathbf{W}} \delta_{x, z} \operatorname{tr}\left(t_{z} \varpi, E^{\infty}\right) v^{-a_{E}} \bmod v^{-a_{E}+1} \mathbf{Z}[v]
$$

and (a) follows.

In the setup of (b), the action of $\sum_{z \in \mathbf{W}, d \in \mathcal{D}, \mathbf{a}(d)=\mathbf{a}(z)} r_{x, d}^{z} t_{z} \varpi$ on $E^{\infty}$ is $\neq 0$. Hence there exist $z \in \mathbf{c}_{E}, d \in \mathcal{D}$ such that $r_{x, d}^{z} \neq 0$ (so that $z \preceq x$ ). This proves (b).

We show:

(c) If $x \in \mathbf{W}$, then $\operatorname{tr}\left(\tilde{T}_{x \varpi}, E^{v} ;-a_{E}\right)=\operatorname{sgn}(x) \operatorname{tr}\left(t_{x} \varpi, E^{\infty}\right)$ and $\operatorname{tr}\left(\tilde{T}_{x \varpi}, E^{v} ; \tilde{a}\right)$ $=0$ for all $\tilde{a}<-a_{E}$.

We argue by induction on $l(x)$. If $l(x)=0$, we have $x=1$ and $\tilde{T}_{x \varpi}=c_{x \varpi}^{\dagger}$ and the result follows from (a). Assume now that $l(x)>0$. From the definition we have $c_{x \varpi}^{\dagger}=\operatorname{sgn}(x) \tilde{T}(x \varpi)+\xi$ where $\xi \in \sum_{x^{\prime} ; l\left(x^{\prime}\right)<l(x)} v \mathbf{Z}[v] \tilde{T}_{x^{\prime} \varpi}$. The induction hypothesis shows that $\operatorname{tr}\left(\xi, E^{v} ; \tilde{a}\right)=0$ for all $\tilde{a} \leq-a_{E}$. Hence $\operatorname{sgn}(x) \operatorname{tr}\left(\tilde{T}_{x \varpi}, E^{v} ; \tilde{a}\right)=$ $\operatorname{tr}\left(c_{x \varpi}^{\dagger}, E^{v} ; \tilde{a}\right)$ for all $\tilde{a} \leq-a_{E}$; now (c) for $x$ follows from (a).

Using (c) and 43.5(b) we see that

$$
f_{E}^{v} \operatorname{dim} E=\sum_{x \in \mathbf{W}} \operatorname{tr}\left(t_{x} \varpi, E^{\infty}\right)^{2} v^{-2 a_{E}}+\text { strictly higher powers of } v .
$$

Now using 43.5(a) we obtain:

$$
f_{E}^{v}=f_{E}^{\infty} v^{-2 a_{E}}+\text { strictly higher powers of } v \text {. }
$$

Now let $E^{\prime}$ be another object of $\operatorname{Irr}(\tilde{\mathbf{W}})$. We show:

(e) $\sum_{x \in \mathbf{W}} \operatorname{tr}\left(t_{x} \varpi, E^{\infty}\right) \operatorname{tr}\left(t_{x} \varpi, E^{\prime \infty}\right)$ is equal to $f_{E}^{\infty} \operatorname{dim} E$ (if $E, E^{\prime}$ are isomorphic) and is equal to 0 if $\left.E^{\prime}\right|_{\mathbf{Q}[\mathbf{W}]} \neq E_{0}$.

We can assume that $\mathbf{c}_{E^{\prime}}=\mathbf{c}_{E}$ (otherwise, the sum in (e) is 0). Combining 43.5(b) with (c) for $E$ and $E^{\prime}$ and with (d) we see that $v^{-2 a_{E}} \sum_{x \in \mathbf{W}} \operatorname{tr}\left(t_{x} \varpi, E^{\infty}\right) \operatorname{tr}\left(t_{x} \varpi\right.$, $\left.E^{\prime \infty}\right)$, plus a $\mathbf{Z}$-linear combination of strictly higher powers of $v$ is equal to $f_{E}^{\infty} \operatorname{dim} E v^{-2 a_{E}}$ plus a Z-linear combination of strictly higher powers of $v$ (if 
$E, E^{\prime}$ are isomorphic) and is equal to 0 if $\left.E^{\prime}\right|_{\mathbf{Q}[\mathbf{W}]} \neq E_{0}$. Taking coefficients of $v^{-2 a_{E}}$ we obtain (e).

We show:

$$
\epsilon\left(\mathbf{c}_{E}\right)=\mathbf{c}_{E} .
$$

For any $x \in \mathbf{W}$ we have $\operatorname{tr}\left(\epsilon(x), E_{0}\right)=\operatorname{tr}\left(x, E_{0}\right)$. It follows that for any $x \in \mathbf{W}$ we have $\operatorname{tr}\left(t_{\epsilon(x)}, E_{0}^{\infty}\right)=\operatorname{tr}\left(t_{x}, E_{0}^{\infty}\right)$. We can find $x \in \mathbf{c}_{E}$ such that $\operatorname{tr}\left(t_{x}, E_{0}^{\infty}\right) \neq 0$. Then $\operatorname{tr}\left(t_{\epsilon(x)}, E_{0}^{\infty}\right) \neq 0$, hence $\epsilon(x) \in \mathbf{c}_{E}$ and (f) follows.

43.7. Let $(W, S)$ be a Weyl group ( $S$ is the set of simple reflections). Let $\sigma: W \rightarrow$ $W$ be an automorphism of $W$ such that $\sigma(S)=S$ and such that whenever $s \neq s^{\prime}$ in $S$ are in the same orbit of $\sigma$, the product $s s^{\prime}$ has order 2 or 3 . Let $b \in \mathbf{Z}_{>0}$ be such that $\sigma^{b}=1$. Let $\tilde{W}$ be the semidirect product of $W$ with the cyclic group $C$ of order $b$ with generator $\sigma$ so that in $\tilde{W}$ we have the identity $\sigma x \sigma^{-1}=\sigma(x)$ for any $x \in W$. Let $I$ be a $\sigma$-stable subset of $S$ and let $W_{I}$ be the subgroup of $W$ generated by $I$. Let $E$ be a simple $\mathbf{Q}[\tilde{W}]$-module such that $\left.E\right|_{\mathbf{Q}[W]}$ is simple. Let $\tilde{W}_{I}=W_{I} C$, a subgroup of $\tilde{W}$. Let $E_{\overline{\mathbf{Q}}_{l}}=\overline{\mathbf{Q}}_{l} \otimes E$. We show:

(a) The $\overline{\mathbf{Q}}_{l}\left[\tilde{W}_{I}\right]$-module $\left.E_{\overline{\mathbf{Q}}_{l}}\right|_{\tilde{W}_{I}}$ is isomorphic to $\oplus_{j} E_{j}^{\prime}$ where each $E_{j}^{\prime}$ is a $\overline{\mathbf{Q}}_{l}\left[\tilde{W}_{I}\right]$-module and either $E_{j}^{\prime}$ is induced from a $\overline{\mathbf{Q}}_{l}\left[W_{I} C^{\prime}\right]$-module where $C^{\prime}$ is a proper subgroup of $C$, or $\left.E_{j}^{\prime}\right|_{W_{I}}$ is simple and $E_{j}^{\prime}$ is defined over $\mathbf{Q}$.

The general case reduces immediately to the case where $\sigma$ permutes transitively the irreducible components of $W$. In this case we may identify $W$ with $W_{1} \times$ $W_{1} \times \cdots \times W_{1}$ and $S=S_{1} \times S_{1} \times \cdots \times S_{1}$ ( $t$ factors) where $\left(W_{1}, S_{1}\right)$ is an irreducible Weyl group; the automorphism $\sigma$ may be written as $\sigma\left(w_{1}, w_{2}, \ldots, w_{t}\right)=$ $\left(\sigma^{\prime}\left(w_{t}\right), w_{1}, w_{2}, \ldots, w_{t-1}\right), w_{i} \in W_{1}$ where $\sigma^{\prime}$ is an automorphism of $\left(W_{1}, S_{1}\right)$. We have $I=I_{1} \times I_{1} \times \cdots \times I_{1}$ where $I_{1} \subset I$ is $\sigma^{\prime}$-stable. Hence $W_{I}=W_{I_{1}} \times W_{I_{1}} \times$ $\cdots \times W_{I_{1}}$. Note that $b / t \in \mathbf{Z}_{>0}$. Let $\tilde{W}_{1}$ be the semidirect product of $W_{1}$ with the cyclic group $C_{1}$ of order $b / t$ with generator $\sigma^{\prime}$ so that in $\tilde{W}_{1}$ we have the identity $\sigma^{\prime} x_{1} \sigma^{\prime-1}=\sigma^{\prime}\left(x_{1}\right)$ for any $x_{1} \in W_{1}$. We can find a simple $\mathbf{Q}\left[\tilde{W}_{1}\right]$-module $E_{1}$ such that $\left.E_{1}\right|_{W_{1}}$ is simple and such that $E=E_{1} \otimes E_{1} \otimes \cdots \otimes E_{1}$ ( $t$ factors) as a $\mathbf{Q}\left[W_{1}\right]$-module and $\sigma$ acts on $E$ as $e_{1} \nabla e_{2} \otimes \cdots \nabla e_{t} \mapsto \sigma^{\prime}\left(e_{t}\right) \nabla e_{1} \nabla e_{2} \otimes \cdots \nabla e_{t-1}$, $\left(e_{i} \in E_{i}\right)$. Let $\tilde{W}_{I_{1}}=W_{I_{1}} C_{1}$, a subgroup of $\tilde{W}_{1}$.

Assume that (a) holds when $W, S, \sigma, b, I, E$ are replaced by $W_{1}, S_{1}, \sigma^{\prime}, b / t, I_{1}, E_{1}$. Let $E_{1, \overline{\mathbf{Q}}_{l}}=\overline{\mathbf{Q}}_{l} \otimes E_{1}$. Then we can identify $\left.E_{1, \overline{\mathbf{Q}}_{l}}\right|_{\tilde{W}_{I_{1}}}=\bigoplus_{j \in \mathcal{J}} E_{1, j}^{\prime}$ where each $E_{1, j}^{\prime}$ is a $\overline{\mathbf{Q}}_{l}\left[\tilde{W}_{I_{1}}\right]$-module with properties like those of $E_{j}^{\prime}$ in (a). We have $E_{\overline{\mathbf{Q}}_{l}}=$

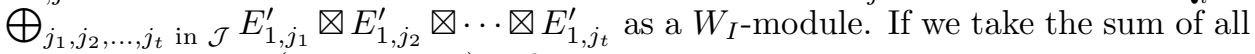
summands where $\left(j_{1}, j_{2}, \ldots, j_{t}\right)$ is fixed up to a cyclic permutation, then we obtain

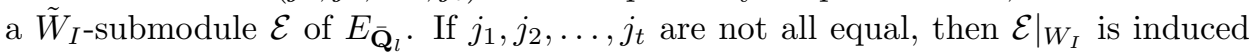
from a $\overline{\mathbf{Q}}_{l}\left[W_{I} C^{\prime}\right]$-module where $C^{\prime}$ is a proper subgroup of $C$. If $j_{1}=j_{2}=\cdots=j_{t}$, then $\mathcal{E}=E_{1, j_{1}}^{\prime} \otimes E_{1, j_{1}}^{\prime} \otimes \cdots \otimes E_{1, j_{1}}^{\prime}$. If, in addition, $E_{1, j_{1}}^{\prime}$ is induced from a $\overline{\mathbf{Q}}_{l}\left[W_{I_{1}} C_{1}^{\prime}\right]$-module where $C_{1}^{\prime}$ is a proper subgroup of $C_{1}$, then $\mathcal{E}$ is a direct sum of $\overline{\mathbf{Q}}_{l}\left[W_{I}\right]$-modules induced from $\overline{\mathbf{Q}}_{l}\left[W_{I} C^{\prime}\right]$-modules where $C^{\prime}$ are proper subgroups of $C$. If, on the other hand, $\left.E_{1, j_{1}}^{\prime}\right|_{W_{I_{1}}}$ is simple and $E_{1, j_{1}}^{\prime}$ is defined over $\mathbf{Q}$, then $\left.\mathcal{E}\right|_{W_{I}}$ is simple and $\mathcal{E}$ is defined over $\mathbf{Q}$. Thus (a) holds for $W, S, \sigma, b, I, E$. We can therefore assume that $(W, S)$ is an irreducible Weyl group. Let $b^{\prime}$ be the order of $\sigma: W \rightarrow W$. We have $b / b^{\prime} \in \mathbf{Z}_{>0}$. By the proof of [L14, 3.2] we can find a $\mathbf{Q}$-linear isomorphism $\sigma^{\prime}: E \rightarrow E$ such that $\sigma^{\prime b^{\prime}}=1$ and $\sigma^{\prime} x \sigma^{\prime-1}=\sigma(x): E \rightarrow E$ for any 
$x \in W$. Since $\left.E\right|_{W}$ is absolutely simple, we must have $\sigma^{\prime}= \pm \sigma: E \rightarrow E$. Hence if (a) holds when $E$ is modified so that the action of $\sigma$ is replaced by that of $\sigma^{\prime}$ (and $b$ is replaced by $b^{\prime}$ ), then (a) also holds for the original $E$ and $b$. Thus we may assume that $b=b^{\prime}$. In this case we have $b \leq 3$. Assume first that $b \leq 2$. We write $\left.E_{\overline{\mathbf{Q}}_{l}}\right|_{\tilde{W}_{I}}=\bigoplus_{j} E_{j}^{\prime}$ where each $E_{j}^{\prime}$ is a simple $\overline{\mathbf{Q}}_{l}\left[\tilde{W}_{I}\right]$-module. If $j$ is such that $\left.E_{j}^{\prime}\right|_{W_{I}}$ is not simple, then $E_{j}^{\prime}$ is induced by a $\overline{\mathbf{Q}}_{l}\left[W_{I} C^{\prime}\right]$-module where $C^{\prime}$ is a proper subgroup of $C$. If $j$ is such that $\left.E_{j}^{\prime}\right|_{W_{I}}$ is simple, then there exists a $\mathbf{Q}\left[W_{I}\right]$-module $E_{0}$ of finite dimension over $\mathbf{Q}$ such that $\left.E_{j}^{\prime}\right|_{W_{I}}=\overline{\mathbf{Q}}_{l} \otimes E_{0}$ as $\overline{\mathbf{Q}}_{l}\left[W_{I}\right]$ modules; moreover, by the proof of [L14, 3.2], there exists a Q-linear isomorphism $\tilde{\sigma}: E_{0} \rightarrow E_{0}$ such that $\tilde{\sigma}^{2}=1$ and $\tilde{\sigma} x \tilde{\sigma}^{-1}=\sigma(x)$ for any $x \in W_{I}$. We extend $\tilde{\sigma}$ to a $\overline{\mathbf{Q}}_{l}$-linear isomorphism $\overline{\mathbf{Q}}_{l} \otimes E_{0} \rightarrow \overline{\mathbf{Q}}_{l} \otimes E_{0}$ denoted again by $\tilde{\sigma}$. Since $E_{0}$ is an absolutely simple $W_{I}$-module we have $\sigma=a \tilde{\sigma}: \overline{\mathbf{Q}}_{l} \otimes E_{0} \rightarrow \overline{\mathbf{Q}}_{l} \otimes E_{0}$ where $a \in \overline{\mathbf{Q}}_{l}^{*}$. Since $\sigma^{2}=\tilde{\sigma}^{2}=1$ on $\overline{\mathbf{Q}}_{l} \otimes E_{0}$, we have $a= \pm 1$. Hence $\sigma: \overline{\mathbf{Q}}_{l} \otimes E_{0} \rightarrow \overline{\mathbf{Q}}_{l} \otimes E_{0}$ is defined over $\mathbf{Q}$. We see that (a) holds for $E$. Next we assume that $b=3$ so that $W$ is of type $D_{4}$. In this case (a) is verified by examining the known explicit $W$-graph realization of $E$. This completes the proof of (a).

43.8. We now return to the setup in 43.1, 43.2. Let $I$ be a subset of $\mathbf{I}$ such that $\epsilon(I)=I$. Let $P \in \mathcal{P}_{I}$ (see 26.1). Then $N_{D} P \neq \emptyset$ so that $D^{\prime}:=N_{D} P / U_{P}$ is a connected component of the reductive group $G^{\prime}:=N_{G} P / U_{P}$; note that $G^{\prime 0}=$ $P / U_{P}$. Let $\tilde{\mathbf{W}}_{I}$ be the subgroup of $\tilde{\mathbf{W}}$ generated by $\mathbf{W}_{I}$ (see 26.1 ) and $\Gamma$; now $\mathbf{W}_{I}, I, \tilde{\mathbf{W}}_{I}$ play the same role for $G^{\prime}, D^{\prime}$ as $\mathbf{W}, \mathbf{I}, \tilde{\mathbf{W}}$ for $G, D$. Let $\tilde{H}_{I}^{v}$ be the algebra defined like $\tilde{H}^{v}$ (with $\mathbf{W}, \mathbf{I}$ replaced by $\mathbf{W}_{I}, I$ ). We have naturally $\tilde{H}_{I}^{v} \subset \tilde{H}^{v}$ as algebras with 1 . For any subgroup $\Gamma^{\prime}$ of $\Gamma$ let $\tilde{H}_{I}^{v, \Gamma^{\prime}}$ be the subspace of $\tilde{H}_{I}^{v}$ spanned by the elements $T_{x \varpi^{i}}$ with $x \in \mathbf{W}_{I}$ and $i \in \mathbf{Z}$ such that $\varpi^{i} \in \Gamma^{\prime}$; this is a subalgebra of $\tilde{H}_{I}^{v}$. Let $\tilde{H}_{I, \overline{\mathbf{Q}}_{l}}^{v}, \tilde{H}_{\overline{\mathbf{Q}}_{l}}^{v}, \tilde{H}_{I, \overline{\mathbf{Q}}_{l}}^{v, \Gamma^{\prime}}$ be the $\overline{\mathbf{Q}}_{l}(v)$-algebras obtained by applying $\overline{\mathbf{Q}}_{l}(v) \otimes_{\mathbf{Q}(v)}()$ to $\tilde{H}_{I}^{v}, \tilde{H}^{v}, \tilde{H}_{I}^{v, \Gamma^{\prime}}$.

Let $E \in \operatorname{Irr}(\tilde{\mathbf{W}})$. Let $E^{v}$ be the $\tilde{H}^{v}$-module corresponding to $E$; see 43.3 . We have the following result:

(a) The restriction to $\tilde{H}_{I, \overline{\mathbf{Q}}_{l}}^{v}$ of the $\tilde{H}_{\overline{\mathbf{Q}}_{l}}^{v}$-module $\overline{\mathbf{Q}}_{l} \otimes E^{v}$ is isomorphic to $\bigoplus_{j} \mathbf{E}_{j}^{\prime}$ where each $\mathbf{E}_{j}^{\prime}$ is a $\tilde{H}_{I, \overline{\mathbf{Q}}_{l}}^{v}$-module and either

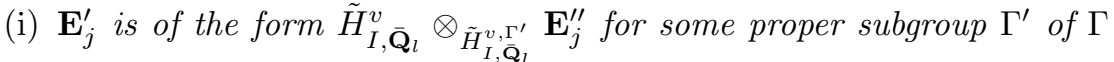
and some $\tilde{H}_{I, \overline{\mathbf{Q}}_{l}}^{v, \Gamma^{\prime}}$-module $\mathbf{E}_{j}^{\prime \prime}$, or

(ii) $\mathbf{E}_{j}^{\prime}$ is of the form $\overline{\mathbf{Q}}_{l} \otimes M_{j}^{v}$ where $M_{j} \in \operatorname{Irr}\left(\tilde{\mathbf{W}}_{I}\right)$.

Here $M_{j}^{v}$ is defined like $E^{v}$ in terms of $\tilde{\mathbf{W}}_{I}$ instead of $\tilde{\mathbf{W}}$.

Note that (a) is a $v$-analogue of $43.7(\mathrm{a})$. It can be proved by the same method as 43.7 (a) or it can be reduced to 43.7 (a) with $W=\mathbf{W}, b=c$.

43.9. In the setup of 43.8 let $x \in \mathbf{W}_{I}$. We show:

$$
\begin{aligned}
\operatorname{tr}\left(\tilde{T}_{x \varpi}, E^{v}\right) & =\sum_{E^{\prime} \in \underline{\operatorname{Ir}}\left(\tilde{\mathbf{W}}_{I}\right)}\left\langle E^{\prime}, E\right\rangle \operatorname{tr}\left(\tilde{T}_{x \varpi}, E^{\prime v}\right), \\
\operatorname{tr}(x \varpi, E) & =\sum_{E^{\prime} \in \underline{\operatorname{Irr}}\left(\tilde{\mathbf{W}}_{I}\right)}\left\langle E^{\prime}, E\right\rangle \operatorname{tr}\left(x \varpi, E^{\prime}\right) ;
\end{aligned}
$$


here for any $E^{\prime}$ in the sum,

$$
\left\langle E^{\prime}, E\right\rangle=\operatorname{dim}_{\mathbf{Q}(v)} \operatorname{Hom}_{\tilde{H}_{I}^{v}}\left(E^{\prime v}, E^{v}\right)=\operatorname{dim}_{\mathbf{Q}} \operatorname{Hom}_{\tilde{\mathbf{W}}_{I}}\left(E^{\prime}, E\right) .
$$

Using 43.8(a) we can write the left-hand side of the first equality in (a) as

$$
\sum_{j} \operatorname{tr}_{\overline{\mathbf{Q}}_{l}(v)}\left(\tilde{T}_{x \varpi}, \mathbf{E}_{j}^{\prime}\right) .
$$

Here $\mathbf{E}_{j}^{\prime}$ is as in $43.8(\mathrm{a})$; if it is as in $43.8(\mathrm{i})$, then $\operatorname{tr}_{\overline{\mathbf{Q}}_{l}(v)}\left(\tilde{T}_{x \varpi}, \mathbf{E}_{j}^{\prime}\right)=0$ since $\Gamma^{\prime} \neq \Gamma$. The contribution of the $j$ as in 43.8(ii) yields the right-hand side of the first equality in (a). The proof of the second equality in (a) is entirely similar.

We show:

(b) If $E^{\prime}$ in (a) satisfies $\left\langle E^{\prime}, E\right\rangle \neq 0$, then $a_{E^{\prime}} \leq a_{E}$;

(here $a_{E}$ is as in 43.6 and $a_{E^{\prime}}$ is defined similarly in terms of $E^{\prime}, \tilde{\mathbf{W}}_{I}$ ). Indeed, the simple $\mathbf{W}_{I}$-module $\left.E^{\prime}\right|_{\mathbf{W}_{I}}$ appears in the $\mathbf{W}_{I}$-module $\left.E\right|_{\mathbf{W}_{I}}$, hence (b) follows from L12, 20.14(a)].

Let $\tilde{H}_{I}^{\infty}$ be defined like $\tilde{H}^{\infty}$ but for $\tilde{\mathbf{W}}_{I}$ instead of $\tilde{\mathbf{W}}$. For $x \in \mathbf{W}_{I}$ we show:

$$
\operatorname{tr}\left(t_{x \varpi}, E^{\infty}\right)=\sum_{E^{\prime} \in \underline{\operatorname{Irr}}\left(\tilde{\mathbf{W}}_{I}\right) ; a_{E^{\prime}}=a_{E}}\left\langle E^{\prime}, E\right\rangle \operatorname{tr}\left(t_{x \varpi}, E^{\prime \infty}\right) .
$$

(The simple $\mathbf{Q} \otimes H_{I}^{\infty}$-module $E^{\prime \infty}$ is defined like $E^{\infty}$ but for $\tilde{\mathbf{W}}_{I}$ instead of $\tilde{\mathbf{W}}$.) We take the coefficient of $v^{-a_{E}}$ in both sides of the first equality in (a) (they are in $\mathcal{A}$; using $43.6(\mathrm{c})$ we obtain

$$
\operatorname{sgn}(x) \operatorname{tr}\left(t_{x} \varpi, E^{\infty}\right)=\sum_{E^{\prime}}\left\langle E^{\prime}, E\right\rangle \operatorname{tr}\left(\tilde{T}_{x \varpi}, E^{\prime v} ;-a_{E}\right)
$$

where the sum over $E^{\prime}$ is as in (a). By (b) the previous sum can be restricted to the $E^{\prime}$ such that $a_{E^{\prime}} \leq a_{E}$. The contribution of $E^{\prime}$ with $a_{E^{\prime}}<a_{E}$ is 0 by $43.6(\mathrm{c})$ (for $\tilde{\mathbf{W}}_{I}$ ). Thus the sum can be restricted to the $E^{\prime}$ such that $a_{E^{\prime}}=a_{E}$. For such $E^{\prime}$ we have, using again 43.6 (for $\tilde{\mathbf{W}}_{I}$ ):

$$
\operatorname{tr}\left(\tilde{T}_{x \varpi}, E^{\prime v} ;-a_{E}\right)=\operatorname{tr}\left(\tilde{T}_{x \varpi}, E^{\prime v} ;-a_{E^{\prime}}\right)=\operatorname{sgn}(x) \operatorname{tr}\left(t_{x} \varpi, E^{\prime \infty}\right)
$$

and (c) follows.

43.10. For any $E \in \operatorname{Irr}(\tilde{\mathbf{W}})$ we define $\phi_{E}: \mathbf{W} \varpi \rightarrow \mathbf{Z}$ by $\phi_{E}(x \varpi)=\operatorname{tr}(x \varpi, E)$. Note that $\phi_{E \otimes \iota}=-\phi_{E}\left(\iota\right.$ as in 43.1). The functions $\phi_{E}$ with $E \in \operatorname{Irr}(\tilde{\mathbf{W}})$ generate a subgroup $\mathcal{R}(\tilde{\mathbf{W}})$ of the group of all functions $\mathbf{W} \varpi \rightarrow \mathbf{Z}$ which are constant on the orbits of the conjugation $\mathbf{W}$-action on $\mathbf{W} \varpi$. From $43.5(\mathrm{c})$ we see that $\left\{\phi_{E} ; E \in \mathfrak{E}\right\}$ is a $\mathbf{Z}$-basis of $\mathcal{R}(\tilde{\mathbf{W}})$. For any $x \in \mathbf{W}$ we set:

$$
\aleph_{x \varpi}=\sum_{E \in \underline{\operatorname{Ir}}(\tilde{\mathbf{W}})} \frac{1}{2} \operatorname{tr}\left(t_{x} \varpi, E^{\infty}\right) \phi_{E}=\sum_{E \in \mathfrak{E}} \operatorname{tr}\left(t_{x} \varpi, E^{\infty}\right) \phi_{E} \in \mathcal{R}(\tilde{\mathbf{W}}) .
$$

From $43.6(\mathrm{e})$ we see that for any $E \in \mathfrak{E}$ we have:

$$
\sum_{x \in \mathbf{W}} \operatorname{tr}\left(t_{x} \varpi, E^{\infty}\right) \aleph_{x \varpi}=f_{E}^{\infty} \operatorname{dim}(E) \phi_{E} \in \mathcal{R}(\tilde{\mathbf{W}}) .
$$


Now let $I$ be a subset of $\mathbf{I}$ such that $\epsilon(I)=I$. We define a homomorphism $J_{\tilde{\mathbf{W}}_{I}}^{\tilde{\tilde{W}}}$ : $\mathcal{R}\left(\tilde{\mathbf{W}}_{I}\right) \rightarrow \mathcal{R}(\tilde{\mathbf{W}})$ by

$$
J_{\tilde{\mathbf{W}}_{I}}^{\tilde{\mathbf{W}}}\left(\phi_{E^{\prime}}\right)=\sum_{E \in \underline{\operatorname{Irr}}(\tilde{\mathbf{W}}) ; a_{E^{\prime}}=a_{E}}\left\langle E^{\prime}, E\right\rangle \phi_{E}
$$

for any $E^{\prime} \in \operatorname{Irr}\left(\tilde{\mathbf{W}}_{I}\right)$. This is clearly well defined. For $x \in \mathbf{W}_{I}$ we define $\aleph_{x \varpi}^{I} \in$ $\mathcal{R}\left(\tilde{\mathbf{W}}_{I}\right)$ in the same way as $\aleph_{x \varpi} \in \mathcal{R}(\tilde{\mathbf{W}})$ but in terms of $\tilde{\mathbf{W}}_{I}$ instead of $\tilde{\mathbf{W}}$. From 43.9 (c) we see that

$$
J_{\tilde{\mathbf{W}}_{I}}^{\tilde{\tilde{W}}}\left(\aleph_{x \varpi}^{I}\right)=\aleph_{x \varpi}^{I}
$$

43.11. Let $I$ be a subset of $\mathbf{I}$ such that $\epsilon(I)=I$. We fix a two-sided cell $\mathbf{c}^{\prime}$ of $\mathbf{W}_{I}$ such that $\epsilon\left(\mathbf{c}^{\prime}\right)=\mathbf{c}^{\prime}$. There is a unique two-sided cell $\mathbf{c}$ of $\mathbf{W}$ such that $\mathbf{c}^{\prime} \subset \mathbf{c}$; we must have $\epsilon(\mathbf{c})=\mathbf{c}$. We show:

(a) if $E^{\prime} \in \operatorname{Irr}\left(\tilde{\mathbf{W}}_{I}\right), E \in \operatorname{Irr}(\tilde{\mathbf{W}})$ satisfy $\mathbf{c}^{\prime}=\mathbf{c}_{E^{\prime}}$ (see 43.6 with $\tilde{\mathbf{W}}$ replaced by $\left.\tilde{\mathbf{W}}_{I}\right)$ and $\left\langle E^{\prime}, E\right\rangle \neq 0$, then $\mathbf{c}_{E} \preceq \mathbf{c}$.

To prove this we may replace $E, E^{\prime}$ by their restrictions to $\mathbf{W}, \mathbf{W}_{I}$. Thus we may assume that $\tilde{\mathbf{W}}=\mathbf{W}, \tilde{\mathbf{W}}_{I}=\mathbf{W}_{I}, \varpi=1$. Since $\mathbf{c}^{\prime}=\mathbf{c}_{E^{\prime}}$, there exists $x \in \mathbf{c}^{\prime}$ such that the action of $t_{x}$ in the $\mathbf{Q} \otimes \tilde{H}_{I}^{\infty}$-module $E^{\prime \infty}$ is $\neq 0$. Using 43.6(a) we see that the action of $c_{x}^{\dagger}$ in the $H_{I}^{v}$-module $E^{\prime v}$ is $\neq 0$. Since $\left\langle E^{\prime}, E\right\rangle \neq 0, E_{v}^{\prime}$ may be regarded as a $H_{I}^{v}$-submodule of $E^{v}$. Hence the action of $c_{x}^{\dagger}$ in the $H^{v}$-module $E^{v}$ is $\neq 0$. Using 43.6 (b) we see that $z \preceq x$ for some $z \in \mathbf{c}_{E}$. By definition we have $x \in \mathbf{c}$. This proves (a).

We show:

(b) if $E^{\prime} \in \operatorname{Irr}\left(\tilde{\mathbf{W}}_{I}\right), E \in \operatorname{Irr}(\tilde{\mathbf{W}})$ satisfy $\mathbf{c}^{\prime}=\mathbf{c}_{E^{\prime}}$ (see 43.6 with $\tilde{\mathbf{W}}$ replaced by $\left.\tilde{\mathbf{W}}_{I}\right)$ and $a_{e^{\prime}}=a_{E},\left\langle E^{\prime}, E\right\rangle \neq 0$, then $\mathbf{c}=\mathbf{c}_{E}$.

Since the a-function of $\mathbf{W}_{I}$ is known to be the restriction of the $\mathbf{a}$-function of $\mathbf{W}$, we see that the value of the $\mathbf{a}$-function on $\mathbf{c}$ and $\mathbf{c}_{E}$ coincide. Since $\mathbf{c}_{E} \preceq \mathbf{c}$ (see (a)) it follows that $\mathbf{c}=\mathbf{c}_{E}$.

43.12. Let $x \in \mathbf{W}$. Let $\mathbf{c}$ be the two-sided cell containing $x$. According to L14, (5.3.1)] there exists uniquely defined elements $a_{y, x} \in \mathbf{Q}(v)$ (for $y \in \mathbf{W}, y \prec x$ ) such that $(-1)^{l(x)} c_{x}^{\dagger}-\sum_{y ; y \prec x}(-1)^{l(y)} a_{y, x} c_{y}^{\dagger}$ acts as zero on $E_{0}^{v}$ for any $E_{0} \in \operatorname{Irr}(\mathbf{W})$ with $\mathbf{c}_{E_{0}} \neq \mathbf{c}$.

Moreover, for $y \prec x$ we have

$$
a_{y, x}=\sum_{j \in \mathbf{Z}_{>0}} a_{y, x ; j} v^{j}
$$

where $a_{y, x ; j} \in \mathbf{Z}$ for all $j$ and $a_{y, x ; j}=0$ unless $j=l(x)+l(y) \bmod 2$; see L14, (5.3.6)]. It follows that the sum

$$
\sum_{E \in \underline{\operatorname{Ir}}(\tilde{\mathbf{W}})} \frac{1}{2} \operatorname{tr}\left(c_{x \varpi}^{\dagger}-\sum_{y ; y \prec x}(-1)^{-l(x)+l(y)} a_{y, x} c_{y \varpi}^{\dagger}, E^{v}\right) \phi_{E} \in \mathcal{R}(\tilde{\mathbf{W}})
$$

is equal to the same sum restricted to those $E$ such that $\mathbf{c}_{E}=\mathbf{c}$. For such $E$ we have $a_{E}=\mathbf{a}(x)$ and for any $y$ such that $y \prec x, a_{y, x} \operatorname{tr}\left(c_{y \varpi}^{\dagger}, E^{v}\right)$ is of the form $v^{-\mathbf{a}(x)+1}$ 
times a rational function in $v$ which is regular at $v=0$; moreover, $\operatorname{tr}\left(c_{x \varpi}^{\dagger}, E^{v}\right)$ is of the form $v^{-\mathbf{a}(x)} \operatorname{tr}\left(t_{x \varpi}, E^{\infty}\right)$ plus higher powers of $v$. Thus (a) is of the form

$$
\sum_{E \in \underline{\operatorname{Ir}}(\tilde{\mathbf{W}}) ; \mathbf{c}_{E}=\mathbf{c}} \frac{1}{2} v^{-\mathbf{a}(x)} \operatorname{tr}\left(t_{x} \varpi, E^{\infty}\right) \phi_{E}+\sigma
$$

where $\sigma$ is a linear combination of elements $\phi_{E}$ with coefficients of the form $v^{-\mathbf{a}(x)+1}$ times a rational function in $v$ which is regular at $v=0$. In the previous sum the condition $\mathbf{c}_{E}=\mathbf{c}$ can be dropped and the sum is unchanged. We see that (a) is equal to $v^{-\mathbf{a}(x)} \aleph_{x \varpi}+\sigma$ with $\sigma$ as above. Taking in this identity coefficients of $v^{-\mathbf{a}(x)}$ in the expansions at $v=0$ we obtain:

$$
\begin{aligned}
\aleph_{x \varpi}= & \sum_{E \in \underline{\operatorname{Irr}}(\tilde{\mathbf{W}})} \frac{1}{2}\left(\operatorname{tr}\left(c_{x \varpi}^{\dagger}, E^{v} ;-\mathbf{a}(x)\right)\right. \\
& \left.-\sum_{y, j ; y \prec x, j>0}(-1)^{-l(x)+l(y)} a_{y, x ; j} \operatorname{tr}\left(c_{y \varpi}^{\dagger}, E^{v} ;-\mathbf{a}(x)-j\right)\right) \phi_{E} .
\end{aligned}
$$

\section{UNIPOTENT CHARACTER SHEAVES AND TWO-SIDED CELLS}

44.1. In this section we study the unipotent character sheaves in connection with Weyl group representations and two-sided cells. A number of results in this section are conditional (they depend on a cleanness property and/or on a parity property); they will become unconditional in $\S 46$.

The following convention will be used in this section. In parts of 44.3-44.7, marked as $\mathbf{p}$...., we assume that the ground field $\mathbf{k}$ is an algebraic closure of $\mathbf{F}_{q}$ and we fix an $\mathbf{F}_{q}$-structure on $G$ with Frobenius map $F: G \rightarrow G$ which leaves $B^{*}, T$ (see 28.5) stable and induces the identity map on $\mathbf{W}$ and on $G / G^{0}$; we will view the various varieties which appear with the natural $\mathbf{F}_{q}$-structure induced by that of $G$. The results in other parts of this section are valid for a general $\mathbf{k}$ (by a standard reduction to the case $\mathbf{k}=\overline{\mathbf{F}}_{q}$ ).

If $X$ is an algebraic variety with a given $\mathbf{F}_{q}$-structure, we write $\mathcal{D}_{m}(X)$ for the corresponding mixed derived category of $\overline{\mathbf{Q}}_{l}$-sheaves. If $A \in \mathcal{D}_{m}(X)$ is perverse and $j \in \mathbf{Z}$, we denote by $A_{j}$ the canonical subquotient of $A$ which is pure of weight $j$.

44.2. For any $w \in \mathbf{W}$ let

$$
Z_{\emptyset, \mathbf{I}, D}^{w}=\left\{\left(B, B^{\prime}, x\right) \in \mathcal{B} \times \mathcal{B} \times D ; x B x^{-1}=B^{\prime}, \operatorname{pos}\left(B, B^{\prime}\right)=w\right\}
$$

(see 28.8),

$$
\bar{Z}_{\emptyset, \mathbf{I}, D}^{w}=\left\{\left(B, B^{\prime}, x\right) \in \mathcal{B} \times \mathcal{B} \times D ; x B x^{-1}=B^{\prime}, \operatorname{pos}\left(B, B^{\prime}\right) \leq w\right\} ;
$$

note that $\bar{Z}_{\emptyset, \mathbf{I}, D}^{w}=\bigsqcup_{w^{\prime} \in \mathbf{W} ; w^{\prime} \leq w} Z_{\emptyset, \mathbf{I}, D}^{w^{\prime}}$. Let

$$
\begin{aligned}
& \mathcal{B}^{w}=\left\{\left(B, B^{\prime}\right) \in \mathcal{B} \times \mathcal{B} ; \operatorname{pos}\left(B, B^{\prime}\right)=w\right\}, \\
& \overline{\mathcal{B}}^{w}=\left\{\left(B, B^{\prime}\right) \in \mathcal{B} \times \mathcal{B} ; \operatorname{pos}\left(B, B^{\prime}\right) \leq w\right\} .
\end{aligned}
$$

Define $\mu: \bar{Z}_{\emptyset, \mathbf{I}, D}^{w} \rightarrow \overline{\mathcal{B}}^{w}$ by $\mu\left(B, B^{\prime}, x\right)=\left(B, B^{\prime}\right)$. Note that $\mu$ is a fibration with connected smooth fibres and $Z_{\emptyset, \mathbf{I}, D}^{w^{\prime}}=\mu^{-1}\left(\mathcal{B}^{w^{\prime}}\right)$ for any $w^{\prime} \leq w$. Hence $Z_{\emptyset, \mathbf{I}, D}^{w}$ is an irreducible smooth open dense subvariety of $\bar{Z}_{\emptyset, \mathbf{I}, D}^{w}$. Let $\overline{\mathbf{Q}}_{l}^{w}$ be the local system 
$\overline{\mathbf{Q}}_{l}$ on $\mathcal{B}^{w}$ and let $\overline{\mathbf{Q}}_{l}^{w \sharp}=I C\left(\overline{\mathcal{B}}^{w}, \overline{\mathbf{Q}}_{l}^{w}\right) \in \mathcal{D}\left(\overline{\mathcal{B}}^{w}\right)$. Let $\dot{\overline{\mathbf{Q}}}^{w}$ be the local system $\overline{\mathbf{Q}}_{l}$ on $Z_{\emptyset, \mathbf{I}, D}^{w}$ and let

$$
\dot{\overline{\mathbf{Q}}}^{w \sharp}=I C\left(\bar{Z}_{\emptyset, \mathbf{I}, D}^{w}, \dot{\overline{\mathbf{Q}}}^{w}\right)=\mu^{*} \overline{\mathbf{Q}}_{l}^{w \sharp} \in \mathcal{D}\left(\bar{Z}_{\emptyset, \mathbf{I}, D}^{w}\right) .
$$

44.3. For $y, w \in \mathbf{W}, y \leq w$ and $i \in \mathbf{Z}$ let $n_{y, w, i}$ be as in 43.2; by [KL2],

(a) $\left.\mathcal{H}^{i}\left(\overline{\mathbf{Q}}_{l}^{w \sharp}\right)\right|_{\mathcal{B}^{y}}$ is a local system isomorphic to $\left(\overline{\mathbf{Q}}_{l}^{y}\right)^{\oplus n_{y, w, i}} ;$ moreover, it admits a filtration (over $\mathbf{F}_{q}$ ) with $n_{y, w, i}$ steps and each subquotient isomorphic over $\mathbf{F}_{q}$ to $\overline{\mathbf{Q}}_{l}(-i / 2)$.

Using the fibration $\mu$ we deduce that

(b) $\left.\mathcal{H}^{i}\left(\dot{\overline{\mathbf{Q}}}^{w \sharp}\right)\right|_{Z_{\emptyset, \mathbf{I}, D}^{y}}$ is a local system isomorphic to $\left(\dot{\overline{\mathbf{Q}}}^{y}\right)^{\oplus n_{y, w, i}}$; moreover, it admits a filtration (over $\mathbf{F}_{q}$ ) with $n_{y, w, i}$ steps and each subquotient isomorphic over $\mathbf{F}_{q}$ to $\overline{\mathbf{Q}}_{l}(-i / 2)$.

Define $\pi_{w}: Z_{\emptyset, \mathbf{I}, D}^{w} \rightarrow D, \bar{\pi}_{w}: \bar{Z}_{\emptyset, \mathbf{I}, D}^{w} \rightarrow D$ by $\left(B, B^{\prime}, x\right) \mapsto x$. Let

$$
K_{D}^{w}=\pi_{w !} \dot{\mathbf{Q}}^{w} \in \mathcal{D}(D), \quad \bar{K}_{D}^{w}=\bar{\pi}_{! w} \dot{\overline{\mathbf{Q}}}^{w \sharp} \in \mathcal{D}(D) .
$$

(With the notation of 28.12 we have $K_{D}^{w}=K_{\mathbf{I}, D}^{w, \overline{\mathbf{Q}}_{l}}$.) We view $\dot{\overline{\mathbf{Q}}}^{w}$ and $\dot{\overline{\mathbf{Q}}}^{w \sharp}$ as objects of $\mathcal{D}_{m}\left(Z_{\emptyset, \mathbf{I}, D}^{w}\right)$ and $\mathcal{D}_{m}\left(\bar{Z}_{\emptyset, \mathbf{I}, D}^{w}\right)$ such that Frobenius acts trivially on the stalk at any $\mathbf{F}_{q}$-rational point of $Z_{\emptyset, \mathbf{I}, D}^{w}$. Applying to them $\pi_{w !}$ and $\bar{\pi}_{! w}$ we obtain objects $\underline{K}_{D}^{w} \in \mathcal{D}_{m}(D), \underline{\bar{K}}_{D}^{w} \in \mathcal{D}_{m}(D)$.

The following equality in the Grothendieck group of mixed perverse sheaves on $D$ is verified (using (b)) along the lines of [L3, 12.6]:

$$
\sum_{i \in \mathbf{Z}}(-1)^{i} H^{i}\left(\underline{\bar{K}}_{D}^{w}\right)=\sum_{y \in \mathbf{W} ; y \leq w} \sum_{i, h \in \mathbf{Z}}(-1)^{i} n_{y, w, h} H^{i}\left(\underline{K}_{D}^{y}\right)(-h / 2) .
$$

We now take the part of weight $j$ in (c); note that $H^{j}\left(\underline{\bar{K}}_{D}^{w}\right)$ is pure of weight $j$ since $\bar{\pi}_{w}$ ! preserve weights and $\dot{\overline{\mathbf{Q}}}^{w \sharp}$ is pure of weight 0 .' We see that for any $j \in \mathbf{Z}$, the following equality holds in the Grothendieck group of perverse sheaves on $D$ :

$$
(-1)^{j} H^{j}\left(\bar{K}_{D}^{w}\right)=\sum_{y \in \mathbf{W} ; y \leq w} \sum_{i, h \in \mathbf{Z}}(-1)^{i} n_{y, w, h} H^{i}\left(\underline{K}_{D}^{y}\right)_{j-h}
$$

44.4. We shall often write $\hat{D}^{u n}$ instead of $\hat{D}^{\overline{\mathbf{Q}}_{l}}$ (see 28.14).

Definition. We say that a character sheaf $A$ on $D$ is unipotent if $A \in \hat{D}^{u n}$.

Let $\underline{D}^{u n}$ be the set of isomorphism classes of unipotent character sheaves on $D$. The following two conditions on a simple perverse sheaf $A$ on $D$ are equivalent:

(i) $A \in \hat{D}^{u n}$.

(ii) $A \dashv \bar{K}_{D}^{w}$ for some $w \in \mathbf{W}$.

This follows from (a) below which is verified along the lines of [L3, (12.7.1)III].

(a) Let $w \in \mathbf{W}$ be such that $A \rtimes K_{D}^{y}$ for any $y \in \mathbf{W}, y<w$. Then $(A$ : $\left.H^{i}\left(\bar{K}_{D}^{w}\right)\right)=\left(A: H^{i}\left(K_{D}^{w}\right)\right)$ for any $i \in \mathbf{Z}$.

Let $\Xi$ be a set of representatives for the isomorphism classes of objects in $\hat{D}^{u n}$; note that $\Xi$ is a finite set. 
44.5. Let $A \in \hat{D}^{u n}$. We regard $H \tilde{T}_{\varpi}$ as an ideal in $\tilde{H}$. Let $\zeta_{0}^{A}: H \tilde{T}_{\varpi} \rightarrow \mathcal{A}$ be the composition of the map $H \tilde{T}_{\varpi} \rightarrow H_{1} \tilde{T}_{\underline{D}}$ (restriction of the natural surjection $\tilde{H} \rightarrow H_{1}^{D}$ ) with the map $\zeta_{A}: H_{1} \tilde{T}_{\underline{D}} \rightarrow \mathcal{A}$ in 31.7 (with $n=1$ ). From the definitions, $\zeta_{A}^{0}$ is an $\mathcal{A}$-linear map and for any $x \in \mathbf{W}$ we have:

$$
\zeta_{0}^{A}\left(v^{l(x)} \tilde{T}_{x \varpi}\right)=v^{-\operatorname{dim} G} \sum_{i, j}(-1)^{i}\left(A: H^{i}\left(\underline{K}_{D}^{x}\right)_{j}\right) v^{j}
$$

For $x \in \mathbf{W}$ we show:

$$
\zeta_{0}^{A}\left(c_{x} \tilde{T}_{\varpi}\right)=v^{-\operatorname{dim} G-l(x)} \sum_{j \in \mathbf{Z}}\left(A: H^{j}\left(\bar{K}_{D}^{x}\right)\right)(-v)^{j} .
$$

a By $44.3(\mathrm{~d})$ we have for any $j$ :

$$
(-1)^{j}\left(A: H^{j}\left(\bar{K}_{D}^{x}\right)\right)=\sum_{y \in \mathbf{W} ; y \leq x} \sum_{i, h \in \mathbf{Z}}(-1)^{i} n_{y, x, h}\left(A: H^{i}\left(\underline{K}_{D}^{y}\right)_{j-h}\right) .
$$

We deduce

$$
\begin{aligned}
& v^{-\operatorname{dim} G-l(x)} \sum_{j \in \mathbf{Z}}\left(A: H^{j}\left(\bar{K}_{D}^{x}\right)\right)(-v)^{j} \\
& =v^{-\operatorname{dim} G-l(x)} \sum_{y \in \mathbf{W} ; y \leq x} \sum_{i, j, h \in \mathbf{Z}}(-1)^{i} n_{y, x, h}\left(A: H^{i}\left(K_{D}^{y}\right)_{j-h}\right) v^{j} \\
& =v^{-\operatorname{dim} G-l(x)} \sum_{y \in \mathbf{W} ; y \leq x} \sum_{i, j^{\prime}, h \in \mathbf{Z}}(-1)^{i} n_{y, x, h}\left(A: H^{i}\left(K_{D}^{y}\right)_{j^{\prime}}\right) v^{j^{\prime}+h}
\end{aligned}
$$

We can rewrite this as

$$
\begin{aligned}
& v^{-l(x)} \sum_{y \in \mathbf{W} ; y \leq x} \sum_{h \in \mathbf{Z}} n_{y, x, h} v^{h} \zeta^{A}\left(v^{l(y)} \tilde{T}_{y \varpi}\right) \\
& =v^{-l(x)} \sum_{y \in \mathbf{W} ; y \leq x} P_{y, x}\left(v^{2}\right) \zeta^{A}\left(v^{l(y)} \tilde{T}_{y \varpi}\right)=\zeta_{0}^{A}\left(c_{x} \tilde{T}_{\varpi}\right) .
\end{aligned}
$$

This proves (b).

44.6. Let $\mathcal{K}^{u n}(D)$ be the subgroup of the Grothendieck group of the category of perverse sheaves on $D$ generated by the objects in $\hat{D}^{u n}$. Let $\mathcal{K}_{\mathbf{Q}}^{u n}(D)=\mathbf{Q} \otimes \mathcal{K}^{u n}(D)$. Let (:) be the symmetric $\mathbf{Q}$-bilinear form on $\mathcal{K}_{\mathbf{Q}}^{u n}(D)$ with values in $\mathbf{Q}$ such that $(A: A)=1$ if $A \in \hat{D}^{u n}$ and $\left(A: A^{\prime}\right)=0$ if $A, A^{\prime} \in \hat{D}^{u n}$ are not isomorphic. Note that if $P$ is a perverse sheaf on $D$ all of whose simple subquotients are in $\hat{D}^{u n}$, then the present meaning of $(A: P)$ agrees with the earlier meaning; see 31.6.

For any $x \in \mathbf{W}$ we show:

$$
g r_{1}\left(\bar{K}_{D}^{x}\right)=\sum_{y \in \mathbf{W} ; y \leq x} P_{y, x}(1) g r_{1}\left(K_{D}^{y}\right) \in \mathcal{K}^{u n}(D) .
$$

Specializing 44.5(c) for $v=1$ we deduce

$$
g r_{1}\left(\bar{K}_{D}^{x}\right)=\sum_{y \in \mathbf{W} ; y \leq x} \sum_{j^{\prime}, h \in \mathbf{Z}} n_{y, x, h} g r_{1}\left(\left(\underline{K}_{D}^{y}\right)_{j^{\prime}}\right) \in \mathcal{K}^{u n}(D)
$$

and (a) follows.

For any $E \in \operatorname{Mod}(\tilde{\mathbf{W}})$ we set

$$
R_{E}=|\mathbf{W}|^{-1} \sum_{x \in \mathbf{W}}(-1)^{\operatorname{dim} G} \operatorname{tr}(x \varpi, E) g r_{1}\left(K_{D}^{x}\right)
$$


(an element of $\left.\mathcal{K}_{\mathbf{Q}}^{u n}(D)\right)$. We show:

$$
R_{E}=|\mathbf{W}|^{-1} \sum_{x \in \mathbf{W}}(-1)^{\operatorname{dim} G} \operatorname{tr}\left(\left.\tilde{c}_{x \varpi}\right|_{v=1}, E\right) g r_{1}\left(\bar{K}_{D}^{x}\right)
$$

where $\tilde{c}_{x \varpi}$ is as in 43.2. We shall use the known inversion formula

$$
\sum_{z \in \mathbf{W} ; y \leq z \leq x}(-1)^{l(y)-l(z)} P_{y, z}(\mathbf{q}) P_{w_{0} x, w_{0} z}(\mathbf{q})=\delta_{y, x}
$$

for any $y \leq x$ in $\mathbf{W}$. Using (a),(d) and the definition of $\tilde{c}_{x \varpi}$, we see that the right-hand side of (c) is

$$
\begin{aligned}
& |\mathbf{W}|^{-1} \sum_{x, y, z \in \mathbf{W} ; y \leq x \leq z}(-1)^{\operatorname{dim} G}(-1)^{l(z)-l(x)} P_{y, x}(1) P_{w_{0} z, w_{0} x}(1) \operatorname{tr}(z \varpi, E) g r_{1}\left(K_{D}^{y}\right) \\
& =|\mathbf{W}|^{-1} \sum_{y \in \mathbf{W}}(-1)^{\operatorname{dim} G} \operatorname{tr}(y \varpi, E) g r_{1}\left(K_{D}^{y}\right)=R_{E}
\end{aligned}
$$

as required.

Let $\operatorname{Mod}_{\overline{\mathbf{Q}}_{l}}(\tilde{\mathbf{W}})$ be the category of $\overline{\mathbf{Q}}_{l}[\tilde{\mathbf{W}}]$-modules of finite dimension over $\overline{\mathbf{Q}}_{l}$. For $E \in \operatorname{Mod}_{\overline{\mathbf{Q}}_{l}}(\tilde{\mathbf{W}})$ we define $R_{E} \in \overline{\mathbf{Q}}_{l} \otimes \mathcal{K}^{u n}(D)$ by the same formula as (b).

For any $\phi \in \mathcal{R}(\tilde{\mathbf{W}})$ (see 43.10 ) we define $R_{\phi} \in \mathcal{K}_{\mathbf{Q}}^{\text {un }}(D)$ by $R_{\phi}=\sum_{E \in \mathfrak{E}} p_{E} R_{E}$ where $\phi=\sum_{E \in \mathfrak{E}} p_{E} \phi_{E}\left(p_{E} \in \mathbf{Z}\right)$. This is independent of the choice of $\mathfrak{E}$ since $R_{E \otimes \iota}=-R_{E}$ for $E \in \operatorname{Irr}(\tilde{\mathbf{W}})$. Note that for $E \in \operatorname{Irr}(\tilde{\mathbf{W}})$ we have $R_{\phi_{E}}=R_{E}$.

44.7. Let $A \in \hat{D}^{u n}$. For any $E \in \operatorname{Irr}(\tilde{\mathbf{W}})$ we set:

$$
b_{A, E}^{v}=\frac{1}{f_{E}^{v} \operatorname{dim} E} \sum_{x \in \mathbf{W}} \zeta_{0}^{A}\left(\tilde{T}_{x \varpi}\right) \operatorname{tr}\left(\tilde{T}_{x \varpi}, E^{v}\right) \in \mathbf{Q}(v) .
$$

Note that this definition is compatible with that in 34.19(b). Using 34.19(a) we see that for any $\xi \in H$ we have

$$
\zeta_{0}^{A}\left(\xi \tilde{T}_{\varpi}\right)=\sum_{E \in \mathfrak{E}} b_{A, E}^{v} \operatorname{tr}\left(\xi \tilde{T}_{\varpi}, E^{v}\right) .
$$

Taking here $\xi=c_{x}, x \in \mathbf{W}$ and using $44.5(\mathrm{~b})$, we deduce:

$$
\sum_{j \in \mathbf{Z}}\left(A: H^{j}\left(\bar{K}_{D}^{x}\right)\right)(-v)^{j}=v^{\operatorname{dim} G+l(x)} \sum_{E \in \mathfrak{E}} b_{A, E}^{v} \operatorname{tr}\left(c_{x} \tilde{T}_{\varpi}, E^{v}\right) .
$$

Let $\hat{D}^{u n c}$ be the subcategory of $\hat{D}^{u n}$ whose objects are the unipotent character sheaves on $D$ which are cuspidal.

An object $A \in \hat{D}^{u n c}$ is said to be clean if the following condition is satisfied: $\left.A\right|_{\bar{S}-S}=0$ where $S$ is the isolated stratum of $D$ such that $\operatorname{supp}(A)$ is the closure $\bar{S}$ of $S$.

We say that $D$ has property $\mathfrak{A}_{0}$ if any $A \in \hat{D}^{u n c}$ is clean. We say that $D$ has property $\mathfrak{A}$ if for any parabolic subgroup $P$ of $G^{0}$ such that $N_{D} P \neq \emptyset$, the connected component $N_{D} P / U_{P}$ of $N_{G} P / U_{P}$ has property $\mathfrak{A}_{0}$. (Compare $33.4(\mathrm{~b})$.)

We say that $D$ has property $\tilde{\mathfrak{A}}$ if for any $A \in \hat{D}^{u n}$ and any $w \in \mathbf{W}, i \in \mathbf{Z}$ such that $\left(A: H^{i}\left(\bar{K}_{D}^{w}\right)\right) \neq 0$ we have $i=\operatorname{dim} \operatorname{supp}(A) \bmod 2$.

In the remainder of this section we assume that $D$ has property $\mathfrak{A}$.

Using $35.18(\mathrm{~g})$ we see that for any $E, E^{\prime}$ in $\mathfrak{E}$ we have

$$
\sum_{A^{\prime} \in \Xi} b_{A^{\prime}, E}^{v} b_{A^{\prime}, E^{\prime}}^{v}=\delta_{E, E^{\prime}}
$$


Let $A \in \hat{D}^{u n}$. Using 35.22 we see that for any $E \in \operatorname{Irr}(\tilde{\mathbf{W}})$ we have

$$
b_{A, E}^{v} \in \mathbf{Q} \text {. }
$$

(The quasi-rationality assumption in 35.22 is automatically satisfied in our case; see 43.4(a).) In view of (e) we shall write $b_{A, E}$ instead of $b_{A, E}^{v}$. We show:

$$
b_{A, E}=(-1)^{\operatorname{dim} G}\left(A: R_{E}\right) .
$$

Let $x \in \mathbf{W}$. Setting $v=1$ in 44.5 (a) we obtain

$$
\left.\zeta_{0}^{A}\left(\tilde{T}_{x \varpi}\right)\right|_{v=1}=\sum_{i, j}(-1)^{i}\left(A: H^{i}\left(\underline{K}_{D}^{x}\right)_{j}\right)=\left(A: g r_{1}\left(K_{D}^{x}\right)\right) .
$$

Setting $v=1$ in (b) with $\xi=\tilde{T}_{x}$ and using (e) we obtain

$$
\left.\zeta_{0}^{A}\left(\tilde{T}_{x \varpi}\right)\right|_{v=1}=\sum_{E \in \mathfrak{E}} b_{A, E} \operatorname{tr}(x \varpi, E) .
$$

Combining with (g) we obtain

$$
\left(A: g r_{1}\left(K_{D}^{x}\right)\right)=\sum_{E \in \mathfrak{E}} b_{A, E} \operatorname{tr}(x \varpi, E) .
$$

Using the orthogonality relations 43.5(b) specialized for $v=1$ we obtain

$$
b_{A, E}=|\mathbf{W}|^{-1} \sum_{x \in \mathbf{W}} \operatorname{tr}(x \varpi, E)\left(A: g r_{1}\left(K_{D}^{x}\right)\right)
$$

for any $E \in \mathfrak{E}$. This proves (f) in the case where $E \in \mathfrak{E}$. This clearly implies (f) in the general case.

We can now rewrite $(\mathrm{h})$ as

$$
g r_{1}\left(K_{D}^{x}\right)=(-1)^{\operatorname{dim} G} \sum_{E \in \mathfrak{E}} \operatorname{tr}(x \varpi, E) R_{E}
$$

in $\mathcal{K}_{\mathbf{Q}}^{u n}(D)$ and (c) as

(j) $\sum_{j \in \mathbf{Z}}\left(A: H^{j}\left(\bar{K}_{D}^{x}\right)\right)(-v)^{j}=(-1)^{\operatorname{dim} G} v^{\operatorname{dim} G+l(x)} \sum_{E \in \mathfrak{E}}\left(A: R_{E}\right) \operatorname{tr}\left(c_{x} \tilde{T}_{\varpi}, E^{v}\right)$.

We show:

(k) there exists $E \in \mathfrak{E}$ such that $\left(A: R_{E}\right) \neq 0$.

We can find $x \in \mathbf{W}$ and $j \in \mathbf{Z}$ such that $\left(A: H^{j}\left(\bar{K}_{D}^{x}\right)\right) \neq 0$. Then the left-hand side of $(\mathrm{j})$ is $\neq 0$, hence so is the right side. Thus (k) holds.

We show:

(l) For $E, E^{\prime} \in \operatorname{Mod}_{\overline{\mathbf{Q}}_{l}}(\tilde{\mathbf{W}})$ we have

$$
\left(R_{E}: R_{E^{\prime}}\right)=|\mathbf{W}|^{-1} \sum_{x \in \mathbf{W}} \operatorname{tr}(x \varpi, E) \operatorname{tr}\left(x \varpi, E^{\prime}\right) .
$$

Moreover, if $E, E^{\prime} \in \mathfrak{E}$, then we have $\left(R_{E}: R_{E^{\prime}}\right)=\delta_{E, E^{\prime}}$.

Here (:) is the bilinear form $\overline{\mathbf{Q}}_{l} \otimes \mathcal{K}^{u n}(D) \times \overline{\mathbf{Q}}_{l} \otimes \mathcal{K}^{u n}(D) \rightarrow \overline{\mathbf{Q}}_{l}$ extending (:) in 44.6 .

Assume first that $E, E^{\prime} \in \mathfrak{E}$. Clearly, $R_{E}=\sum_{A^{\prime} \in \Xi}\left(A^{\prime}: R_{E}\right) A^{\prime}, R_{E^{\prime}}=$ $\sum_{A^{\prime} \in \Xi}\left(A^{\prime}: R_{E^{\prime}}\right) A^{\prime}$. It follows that

$$
\left(R_{E}: R_{E^{\prime}}\right)=\sum_{A^{\prime} \in \Xi}\left(A^{\prime}: R_{E}\right)\left(A^{\prime}: R_{E^{\prime}}\right)=\sum_{A^{\prime} \in \Xi} b_{A^{\prime}, E} b_{A^{\prime}, E^{\prime}}=\delta_{E, E^{\prime}}
$$


where the last two equalities come from (f),(d). This proves the second equality in (l). To prove the first equality in (l) we may assume that $E, E^{\prime}$ are simple objects of $\operatorname{Mod}_{\overline{\mathbf{Q}}_{l}}(\tilde{\mathbf{W}})$. If the restriction of $E$ to $\overline{\mathbf{Q}}_{l}[\mathbf{W}]$ is not simple, then $\operatorname{tr}(x \varpi, E)=0$ for any $x \in \mathbf{W}$, hence both sides of the first equality in (l) are 0 . Thus we may assume in addition that $\left.E\right|_{\overline{\mathbf{Q}}_{l}[\mathbf{W}]}$ is simple; similarly we may assume that $\left.E^{\prime}\right|_{\overline{\mathbf{Q}}_{l}[\mathbf{W}]}$ is simple. Replacing $E, E^{\prime}$ by their tensor products with one-dimensional representations of $\tilde{\mathbf{W}}$ which are trivial on $\mathbf{W}$ reduces us to the case where $E, E^{\prime}$ come from objects of $\mathfrak{E}$ by extension of scalars. Using then the second identity in (l) we see that it is enough to show that for $E, E^{\prime} \in \mathfrak{E}$ we have $|\mathbf{W}|^{-1} \sum_{x \in \mathbf{W}} \operatorname{tr}(x \varpi, E) \operatorname{tr}\left(x \varpi, E^{\prime}\right)=\delta_{E, E^{\prime}}$; but this is known from 43.5(c). This completes the proof of (l).

For any $x \in \mathbf{W}, i \in \mathbf{Z}$ we take the coefficient of $v^{i+l(x)+\operatorname{dim} G}$ in the two sides of (j); we obtain:

$$
(-1)^{i+l(x)}\left(A: H^{i+l(x)+\operatorname{dim} G}\left(\bar{K}_{D}^{x}\right)\right)=\sum_{E \in \underline{\operatorname{Irr}}(\tilde{\mathbf{W}})} \frac{1}{2} \operatorname{tr}\left(c_{x} \tilde{T}_{\varpi}, E^{v} ; i\right)\left(A: R_{E}\right) .
$$

For any $y, z$ in $\mathbf{W}$ we show:

$$
g r_{1}\left(K_{D}^{y^{-1} z \varpi y \varpi^{-1}}\right)=g r_{1}\left(K_{D}^{z}\right)
$$

Using (i) this is the same as

$$
\sum_{E \in \mathfrak{E}} \operatorname{tr}\left(y^{-1} z \varpi y, E\right) R_{E}=\sum_{E \in \mathfrak{E}} \operatorname{tr}(z \varpi, E) R_{E}
$$

which is clear since $\operatorname{tr}\left(y^{-1} z \varpi y, E\right)=\operatorname{tr}(z \varpi, E)$ for any $E \in \mathfrak{E}$.

We show:

(o) If $E \in \operatorname{Mod}(\tilde{\mathbf{W}})$, then $R_{E}$ is a $\mathbf{Z}$-linear combination of elements $R_{E_{1}}$ with $E_{1} \in \operatorname{Irr}(\tilde{\mathbf{W}})$.

We can write $\overline{\mathbf{Q}}_{l} \otimes E=\bigoplus_{h} \mathbf{E}_{h}$ where $\mathbf{E}_{h}$ are simple $\overline{\mathbf{Q}}_{l}[\tilde{\mathbf{W}}]$-modules. Hence $R_{E}=R_{\overline{\mathbf{Q}}_{l} \otimes E}=\sum_{h} R_{\mathbf{E}_{h}}$. If $h$ is such that $\left.\mathbf{E}_{h}\right|_{\mathbf{W}}$ is not a simple $\overline{\mathbf{Q}}_{l}[\mathbf{W}]$-module, then $\operatorname{tr}\left(x \varpi, \mathbf{E}_{h}\right)=0$ for any $x \in \mathbf{W}$, hence $R_{\mathbf{E}_{h}}=0$. If $h$ is such that $\left.\mathbf{E}_{h}\right|_{\mathbf{W}}$ is a simple $\overline{\mathbf{Q}}_{l}[\mathbf{W}]$-module, then by taking the tensor products of $\mathbf{E}_{h}$ with a onedimensional representation of $\tilde{\mathbf{W}}$ which is trivial on $\mathbf{W}$ we obtain a module which comes from an object of $\operatorname{Irr}(\tilde{\mathbf{W}})$. It follows that $R_{E}=\sum_{E_{1} \in \mathfrak{E}} c_{E_{1}} R_{E_{1}}$ where $c_{E_{1}}$ are integer combination of roots of 1 . Using (l) we have $c_{E_{1}}=\left(R_{E}: R_{E_{1}}\right)=$ $|\mathbf{W}|^{-1} \sum_{x \in \mathbf{W}} \operatorname{tr}(x \varpi, E) \operatorname{tr}\left(x \varpi, E_{1}\right)$. This is a rational number; being also an algebraic integer it is an integer. This proves (o).

We show:

(p) For $E \in \mathfrak{E}, x \in \mathbf{W}$ we have $\left(R_{E}: g r_{1}\left(K_{D}^{x}\right)\right)=(-1)^{\operatorname{dim} G} \operatorname{tr}(x \varpi, E)$.

Using (i) we have $\left(R_{E}: g r_{1}\left(K_{D}^{x}\right)\right)=\left(R_{E}:(-1)^{\operatorname{dim} G} \sum_{E^{\prime} \in \mathfrak{E}} \operatorname{tr}\left(x \varpi, E^{\prime}\right) R_{E^{\prime}}\right)$ so that (p) follows from (l).

44.8. The $\mathcal{A}$-linear involution $\mathbf{d}: \mathfrak{K}(D) \rightarrow \mathfrak{K}(D)$ in 42.2 induces (by the specialization $v=1)$ a Z-linear involution $\mathbf{d}: \mathcal{K}(D) \rightarrow \mathcal{K}(D)(\mathcal{K}(D)$ as in 38.9). By extension of scalars, $\mathbf{d}$ gives rise to a $\mathbf{Q}$-linear involution $\mathbf{Q} \otimes \mathcal{K}(D) \rightarrow \mathbf{Q} \otimes \mathcal{K}(D)$ denoted again by $\mathbf{d}$.

Let $A \in \hat{D}$. We show that:

$$
\mathbf{d}(A)=(-1)^{\operatorname{codim}(\operatorname{supp}(A))} A^{\circ}
$$


where $A^{\circ} \in \hat{D}$. We can find a parabolic $P_{0}$ of $G^{0}$ such that $N_{D} P_{0} \neq \emptyset$ and a cuspidal character sheaf $A_{0}$ on $D_{0}:=N_{D} P_{0} / U_{P_{0}}$ such that $A$ is a direct summand of $\operatorname{ind}_{D_{0}}^{D}\left(A_{0}\right)$. We have $P_{0} \in \mathcal{P}_{J}$ where $J \subset \mathbf{I}, \epsilon(J)=J$. By 38.11(a) we have $\mathbf{d}(A)=$ $(-1)^{\left|J_{\epsilon}\right|} A^{\circ}$ where $A^{\circ} \in \hat{D}$ and $J_{\epsilon}$ is the set of orbits of $\epsilon: J \rightarrow J$. It remains to show that $\operatorname{codim}(\operatorname{supp}(A))=\left|J_{\epsilon}\right| \bmod 2$. From the theory of admissible complexes (6.7) and from 3.13(b) we see that $\operatorname{dim} \operatorname{supp}(A)=\operatorname{dim} G^{0}-\operatorname{dim}\left(P_{0} / U_{P_{0}}\right)+\operatorname{dim} \operatorname{supp}\left(A_{0}\right)$; that is, $\operatorname{codim}(\operatorname{supp}(A))=\operatorname{codim}\left(\operatorname{supp}\left(A_{0}\right)\right)$. Also, the analogue of $J_{\epsilon}$ for $A_{0}$ is $J_{\epsilon}$ itself. Thus we are reduced to the case where $A=A_{0}$; that is, we may assume that $A$ is cuspidal. Let $G^{\prime}={ }^{D} \mathcal{Z}_{G^{0}}^{0} \backslash G, D^{\prime}={ }^{D} \mathcal{Z}_{G^{0}}^{0} \backslash D$. Then the support of $A$ is the closure of a subset of $D$ which is the inverse image of a single $G^{\prime 0}$-conjugacy class $C$ in $D^{\prime}$ under the obvious map $D \rightarrow D^{\prime}$. Moreover, ${ }^{D^{\prime}} \mathcal{Z}_{G^{\prime}}^{0}=\{1\}$. The set $\mathbf{I}$ for $G^{\prime}$ can be identified with that for $G$. Since $\operatorname{codim}(\operatorname{supp}(A))=\operatorname{codim}_{D^{\prime}} C$, it is enough to show that $\operatorname{codim}_{D^{\prime}} C=\left|\mathbf{I}_{\epsilon}\right| \bmod 2$ for any $G^{\prime 0}$-conjugacy class $C$ in $D^{\prime}$. According to Spaltenstein $\left[\underline{\mathrm{S}}\right.$ we have $\operatorname{codim}_{D^{\prime}} C=2 \beta+r$ where $\beta$ is the dimension of the variety of Borel subgroups of $G^{\prime 0}$ that are normalized by some fixed element of $C$ and $r$ is the rank of the connected centralizer in $G^{\prime}$ of any quasisemisimple element of $D^{\prime}$. Thus, $\operatorname{codim}_{D^{\prime}} C=r \bmod 2$. It remains to note that $r=\left|\mathbf{I}_{\epsilon}\right|$.

By 42.9 (specialized with $v=1$ ) we see that for any $x \in \mathbf{W}$ we have:

$$
\mathbf{d}\left(\sum_{i \in \mathbf{Z}} H^{i}\left(K_{D}^{x}\right)\right)=(-1)^{l(x)} \sum_{i \in \mathbf{Z}} H^{i}\left(K_{D}^{x}\right)
$$

in $\mathcal{K}(D)$. Here $H^{i}\left(K_{D}^{x}\right)$ is identified with the element $\sum_{A^{\prime} \in \Xi}\left(A^{\prime}: H^{i}\left(K_{D}^{x}\right)\right) A^{\prime}$ of $\mathcal{K}(D)$. We show that for any $E \in \operatorname{Irr}(\tilde{\mathbf{W}})$ we have:

$$
\mathbf{d}\left(R_{E}\right)=R_{E \otimes \mathrm{sgn}} \text {. }
$$

Indeed, by (b), this is the same as the obvious equality

$$
\begin{aligned}
& |\mathbf{W}|^{-1} \sum_{i \in \mathbf{Z}} \sum_{x \in \mathbf{W}}(-1)^{i+\operatorname{dim} G+l(x)} \operatorname{tr}(x \varpi, E) H^{i}\left(K_{D}^{x}\right) \\
& =|\mathbf{W}|^{-1} \sum_{i \in \mathbf{Z}} \sum_{x \in \mathbf{W}}(-1)^{i+\operatorname{dim} G} \operatorname{tr}(x \varpi, E \otimes \operatorname{sgn}) H^{i}\left(K_{D}^{x}\right) .
\end{aligned}
$$

If $A \in \hat{D}^{u n}$, then, by $44.7(\mathrm{k})$, there exists $E \in \operatorname{Irr}(\tilde{\mathbf{W}})$ such that the coefficient of $A$ in $R_{E}$ is $\neq 0$. Applying $\mathbf{d}$ to $R_{E}$ we see that the coefficient of $A^{\circ}$ in $\mathbf{d}\left(R_{E}\right)$ is $\neq 0$; that is, the coefficient of $A^{\circ}$ in $R_{E \otimes s g n}$ is $\neq 0$. In particular, $A^{\circ} \in \hat{D}^{u n}$. In the same way we see that for any $E \in \operatorname{Irr}(\tilde{\mathbf{W}})$ we have

$$
\left(A: R_{E}\right)= \pm\left(A^{\circ}: R_{E \otimes \mathrm{sgn}}\right) \text {. }
$$

Using (a) and the equality $\mathbf{d} \mathbf{d}=1$ we obtain

$$
A=(-1)^{\operatorname{codim}(\operatorname{supp}(A))} \mathbf{d}\left(A^{\circ}\right)=(-1)^{\operatorname{codim}(\operatorname{supp}(A))}(-1)^{\operatorname{codim}\left(\operatorname{supp}\left(A^{\circ}\right)\right)}\left(A^{\circ}\right)^{\circ} .
$$

It follows that $\left(A^{\circ}\right)^{\circ} \cong A$ and

$$
\operatorname{codim}(\operatorname{supp}(A))=\operatorname{codim}\left(\operatorname{supp}\left(A^{\circ}\right)\right) \bmod 2 .
$$

44.9. For any sequence $\mathbf{s}=\left(s_{1}, s_{2}, \ldots, s_{r}\right)$ in $\mathbf{I}$ we write $K_{D}^{\mathbf{s}}, \bar{K}_{D}^{\mathbf{s}}$ instead of $K_{\mathbf{I}, D}^{\mathbf{s}, \overline{\mathbf{Q}}_{l}}$, $\bar{K}_{\mathbf{I}, D}^{\mathbf{s}, \overline{\mathbf{Q}}_{l}} ;$ see 28.12 .

Let $A \in \hat{D}^{u n}$. Then $\left(A: H^{i}\left(\bar{K}_{D}^{w}\right)\right) \neq 0$ for some $w \in \mathbf{W}, i \in \mathbf{Z}$. We set

$$
\mathbf{e}^{A}=(-1)^{i+\operatorname{dim} G} .
$$


We show that $\mathbf{e}^{A}$ is well defined. Assume that we have also $\left(A: H^{i^{\prime}}\left(\bar{K}_{D}^{w^{\prime}}\right)\right) \neq 0$ with $w^{\prime} \in \mathbf{W}, i^{\prime} \in \mathbf{Z}$. We must show that $i=i^{\prime} \bmod 2$. Let $\mathbf{s}=\left(s_{1}, s_{2}, \ldots, s_{r}\right)$, $\mathbf{s}^{\prime}=\left(s_{1}^{\prime}, s_{2}^{\prime}, \ldots, s_{r^{\prime}}^{\prime}\right)$ be sequences in $\mathbf{I}$ such that $s_{1} s_{2} \ldots s_{r}=w, s_{1}^{\prime} s_{2}^{\prime} \ldots s_{r^{\prime}}^{\prime}=w^{\prime}$, $r=l(w), r^{\prime}=l\left(w^{\prime}\right)$. We will show that

(b) $\bar{K}_{D}^{w}$ is a direct summand of $\bar{K}_{D}^{\mathrm{s}}$.

Assuming this and a similar statement for $w^{\prime}, \mathbf{s}^{\prime}$ instead of $w$, $\mathbf{s}$ we see that $(A$ : $\left.H^{i}\left(\bar{K}_{D}^{\mathbf{s}}\right)\right) \neq 0$ and $\left(A: H^{i^{\prime}}\left(\bar{K}_{D}^{\mathbf{s}^{\prime}}\right)\right) \neq 0$ and the congruence $i=i^{\prime} \bmod 2$ follows from 35.17(a). (Although in 35.17 it is assumed that $D$ is clean, in the present application it is enough to use the weaker hypothesis that $\mathfrak{A}$ holds for $D$.)

Recall that $\bar{K}_{D}^{\mathbf{s}}=\bar{\pi}_{\mathbf{s}} ! \overline{\mathbf{Q}}_{l}$ where

$$
\begin{aligned}
\bar{Z}_{\emptyset, \mathbf{I}, D}^{\mathbf{s}}=\left\{\left(B_{0}, B_{1}, \ldots, B_{r}, g\right) \in \mathcal{B}^{r+1} \times D ;\right. \\
\left.g B_{0} g^{-1}=B_{r}, \operatorname{pos}\left(B_{i-1}, B_{i}\right) \in\left\{1, s_{i}\right\} \text { for } i \in[1, r]\right\}
\end{aligned}
$$

and $\bar{\pi}_{\mathbf{s}}: \bar{Z}_{\emptyset, \mathbf{I}, D}^{\mathbf{s}} \rightarrow D$ is given by $\left(B_{0}, B_{1}, \ldots, B_{r}, g\right) \mapsto g$. Recall from 44.2 that $\bar{K}_{D}^{w}=\bar{\pi}_{w !} \dot{\overline{\mathbf{Q}}}^{w \sharp}$. We have $\bar{\pi}_{\mathbf{s}}=\bar{\pi}_{w} \rho$ where $\rho: \bar{Z}_{\emptyset, \mathbf{I}, D}^{\mathbf{s}} \rightarrow \bar{Z}_{\emptyset, \mathbf{I}, D}^{w}$ is given by $\left(B_{0}, B_{1}, \ldots, B_{r}, g\right) \mapsto\left(B_{0}, B_{r}, g\right)$. Hence $\bar{K}_{D}^{\mathbf{s}}=\bar{p}_{w !}\left(\rho_{!} \overline{\mathbf{Q}}_{l}\right)$ so that to prove (b) it is enough to show that $\dot{\overline{\mathbf{Q}}}^{w \sharp}$ is a direct summand of $\rho_{!} \overline{\mathbf{Q}}_{l}$. This follows from the fact that $\rho$ is proper and is an isomorphism over an open dense subset of $\bar{Z}_{\emptyset, \mathbf{I}, D}^{w}$. This proves (b).

44.10. We now fix a subset $I \subset \mathbf{I}$ such that $\epsilon(I)=I$. Let $P \in \mathcal{P}_{I}$ (see 26.1). Then $N_{D} P \neq \emptyset$ so that $D^{\prime}:=N_{D} P / U_{P}$ is a connected component of the reductive group $G^{\prime}:=N_{G} P / U_{P}$; note that $G^{\prime 0}=P / U_{P}$. Let $\pi^{\prime}: N_{D} P \rightarrow D^{\prime}$ be the obvious map. As in 27.1 we consider the diagram $D^{\prime} \stackrel{a}{=} V_{1} \stackrel{a^{\prime}}{\longrightarrow} V_{2} \stackrel{a^{\prime \prime}}{\longrightarrow} D$ where $V_{1}=\left\{(g, x) \in D \times G^{0} ; x^{-1} g x \in N_{D} P\right\}, V_{2}=\left\{(g, x P) \in D \times G^{0} / P ; x^{-1} g x \in N_{D} P\right\}$, $\underline{a}(g, x)=\pi^{\prime}\left(x^{-1} g x\right), a^{\prime}(g, x)=(g, x P), a^{\prime \prime}(g, x P)=g$. As in 27.1 for any $G^{\prime 0}$ equivariant perverse sheaf $A^{\prime}$ we define a complex of sheaves $A=\operatorname{ind}_{D^{\prime}}^{D}\left(A^{\prime}\right) \in \mathcal{D}(D)$ by $A=a_{!}^{\prime \prime} A_{1}^{\prime}\left[2 \operatorname{dim} U_{P}\right]$ where $A_{1}^{\prime} \in \mathcal{D}\left(V_{2}\right)$ is such that $\underline{a}^{*} A^{\prime}=a^{\prime *} A_{1}^{\prime}$. We show:

(a) If $A^{\prime} \in \hat{D}^{\prime u n}$, then $\operatorname{ind}_{D^{\prime}}^{D}\left(A^{\prime}\right)$ is isomorphic to a direct sum of objects of $\hat{D}^{u n}$.

The proof is similar to that of [L3, 4.8(I)]. Before giving it we need some preliminaries. Let $\mathcal{B}^{\prime}$ be the flag manifold of $G^{\prime 0}=P / U_{P}$. For $\beta \in \mathcal{B}^{\prime}$ let $\tilde{\beta} \in \mathcal{B}$ be the inverse image of $\beta$ under the obvious map $P \rightarrow G^{\prime 0}$. Let $w \in \mathbf{W}_{I}$ (see 26.1). Recall that

$$
\bar{Z}_{\emptyset, \mathbf{I}, D}^{w}=\left\{\left(B, B^{\prime}, x\right) \in \mathcal{B} \times \mathcal{B} \times D ; x B x^{-1}=B^{\prime}, \operatorname{pos}\left(B, B^{\prime}\right) \leq w\right\} .
$$

Replacing here $D, \mathbf{I}$ by $D^{\prime}, I$ we have

$$
\bar{Z}_{\emptyset, I, D^{\prime}}^{w}=\left\{\left(\beta, \beta^{\prime}, y\right) \in \mathcal{B}^{\prime} \times \mathcal{B}^{\prime} \times D^{\prime} ; y \beta y^{-1}=\beta^{\prime}, \operatorname{pos}\left(\beta, \beta^{\prime}\right) \leq w\right\} .
$$

We have a commutative diagram with cartesian squares

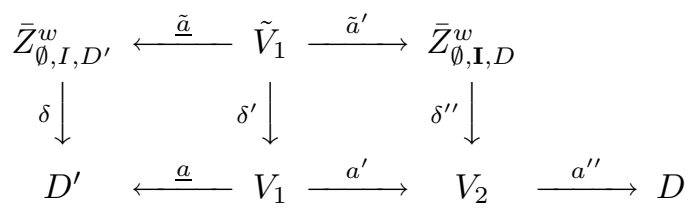


where

$$
\begin{array}{r}
\tilde{V}_{1}=\left\{\left(\beta, b^{\prime}, y, g, x\right) \in \mathcal{B}^{\prime} \times \mathcal{B}^{\prime} \times D^{\prime} ; y \beta y^{-1}=\beta^{\prime}, x^{-1} g x \in N_{D} P,\right. \\
\left.y=\pi^{\prime}\left(x^{-1} g x\right), \operatorname{pos}\left(\beta, \beta^{\prime}\right) \leq w\right\}, \\
\underline{\tilde{a}}\left(\beta, \beta^{\prime}, y, g, x\right)=\left(\beta, \beta^{\prime}, y\right), \tilde{a}^{\prime}\left(\beta, \beta^{\prime}, y, g, x\right)=\left(x \tilde{\beta} x^{-1}, x \tilde{\beta}^{\prime} x^{-1}, g\right), \\
\delta\left(\beta, \beta^{\prime}, y\right)=y, \delta^{\prime}\left(\beta, \beta^{\prime}, y, g, x\right)=(g, x), \delta^{\prime \prime}\left(B, B^{\prime}, x\right)=(x, z P)
\end{array}
$$

with $z \in G^{0}$ such that $z^{-1} B z \subset P$.

Note that $\underline{a}, \underline{\tilde{a}}$ are smooth with connected fibres and $\tilde{a}^{\prime}, a^{\prime}$ are principal $P$ bundles. It follows that

$$
I C\left(\tilde{V}_{1}, \overline{\mathbf{Q}}_{l}\right)=\underline{\tilde{a}}^{*} I C\left(\bar{Z}_{\emptyset, I, D^{\prime}}^{w}, \overline{\mathbf{Q}}_{l}\right)=\tilde{a}^{\prime *} I C\left(\bar{Z}_{\emptyset, \mathbf{I}, D}^{w}, \overline{\mathbf{Q}}_{l}\right)
$$

where the first $\overline{\mathbf{Q}}_{l}$ lives on

$$
\left\{\left(\beta, b^{\prime}, y, g, x\right) \in \tilde{V}_{1} ; \operatorname{pos}\left(\beta, \beta^{\prime}\right)=w\right\}=\underline{\tilde{a}}^{-1}\left(Z_{\emptyset, I, D^{\prime}}^{w}\right)=\tilde{a}^{\prime-1}\left(Z_{\emptyset, \mathbf{I}, D}^{w}\right),
$$

the second $\overline{\mathbf{Q}}_{l}$ lives on $Z_{\emptyset, I, D^{\prime}}^{w}$ and the third $\overline{\mathbf{Q}}_{l}$ lives on $Z_{\emptyset, \mathbf{I}, D}^{w}$. Hence

$$
\delta_{!}^{\prime} I C\left(\tilde{V}_{1}, \overline{\mathbf{Q}}_{l}\right)=\underline{a}^{*} \delta_{!} I C\left(\bar{Z}_{\emptyset, I, D^{\prime}}^{w}, \overline{\mathbf{Q}}_{l}\right)=\alpha^{\prime *} \delta_{!}^{\prime \prime} I C\left(\bar{Z}_{\emptyset, \mathbf{I}, D}^{w}, \overline{\mathbf{Q}}_{l}\right) ;
$$

that is, $\delta_{!}^{\prime} I C\left(\tilde{V}_{1}, \overline{\mathbf{Q}}_{l}\right)=\underline{a}^{*} \bar{K}_{D^{\prime}}^{w}=\alpha^{*} K^{\prime}$ where $K^{\prime}=\delta_{!}^{\prime \prime} I C\left(\bar{Z}_{\emptyset, \mathbf{I}, D}^{w}, \overline{\mathbf{Q}}_{l}\right) \in \mathcal{D}\left(V_{2}\right)$. Since $\underline{a}, a^{\prime}$ are smooth with connected fibres of $\operatorname{dimension} \operatorname{dim} D+\operatorname{dim} U_{P}, \operatorname{dim} D-$ $\operatorname{dim} U_{P}$, respectively, we see that for any $i$ we have

$$
\begin{aligned}
& \underline{a}^{*}\left(H^{i-\operatorname{dim} D-\operatorname{dim} U_{P}} \bar{K}_{D^{\prime}}^{w}\right)\left[\operatorname{dim} D+\operatorname{dim} U_{P}\right]=H^{i}\left(\underline{a}^{*} \bar{K}_{D^{\prime}}^{w}\right) \\
& =H^{i}\left(a^{\prime *} \bar{K}_{D}^{w}\right)=a^{\prime *}\left(H^{i-\operatorname{dim} D+\operatorname{dim} U_{P}} K^{\prime}\right)\left[\operatorname{dim} D-\operatorname{dim} U_{P}\right],
\end{aligned}
$$

hence $\left(\operatorname{setting} j=i-\operatorname{dim} D-\operatorname{dim} U_{P}\right)$ :

$$
\underline{a}^{*}\left(H^{j} \bar{K}_{D^{\prime}}^{w}\right)=a^{\prime *}\left(H^{j+2 \operatorname{dim} U_{P}} K^{\prime}\right)\left[-2 \operatorname{dim} U_{P}\right] .
$$

We see that

$$
\operatorname{ind}_{D^{\prime}}^{D}\left(H^{j} \bar{K}_{D^{\prime}}^{w}\right)=a_{!}^{\prime \prime}\left(H^{j+2 \operatorname{dim} U_{P}} K^{\prime}\right) .
$$

We have

$$
\bigoplus_{j} \operatorname{ind}_{D^{\prime}}^{D}\left(H^{j} \bar{K}_{D^{\prime}}^{w}\right)[-j]=\bigoplus_{j}\left(H^{j+2 \operatorname{dim} U_{P}} \bar{K}_{D}^{w}\right)[-j] \text { in } \mathcal{D}(D) .
$$

Indeed the left-hand side is

$$
\begin{aligned}
& \bigoplus_{j} a_{!}^{\prime \prime}\left(H^{j+2 \operatorname{dim} U_{P}} K^{\prime}\right)=a_{!}^{\prime \prime} K^{\prime}\left[2 \operatorname{dim} U_{P}\right] \\
& =a_{!}^{\prime \prime} \delta_{!}^{\prime \prime} I C\left(\bar{Z}_{\emptyset, \mathbf{I}, D}^{w}, \overline{\mathbf{Q}}_{l}\right)\left[2 \operatorname{dim} U_{P}\right]=\bar{K}_{D}^{w}\left[2 \operatorname{dim} U_{P}\right] ;
\end{aligned}
$$

(we have used that $K^{\prime} \cong \bigoplus_{j} H^{j} K^{\prime}[-j]$ which follows from the decomposition theorem [BBD] applied to the proper map $\left.\delta^{\prime \prime}\right)$. This is equal to the right-hand side of (b) since $\bar{K}_{D}^{w} \cong \bigoplus_{j} H^{j}\left(\bar{K}_{d}^{w}\right)[-j]$, by the decomposition theorem applied to the proper map $a^{\prime \prime} \delta^{\prime \prime}$. Now $H^{j} \bar{K}_{D^{\prime}}^{w}$ is a direct sum of character sheaves on $D^{\prime}$; hence, by $30.6(\mathrm{a})$, ind $D_{D^{\prime}}^{D}\left(H^{j} \bar{K}_{D^{\prime}}^{w}\right)$ is a perverse sheaf on $D$ for any $j$. Taking $H^{i}$ for both sides of (b) we obtain for any $i \in \mathbf{Z}$ :

$$
\operatorname{ind}_{D^{\prime}}^{D}\left(H^{i} \bar{K}_{D^{\prime}}^{w}\right)=H^{i+2 \operatorname{dim} U_{P}} \bar{K}_{D}^{w}
$$

Now let $A^{\prime} \in \hat{D}^{\prime u n}$. We can find $w \in \mathbf{W}_{I}$ and $i \in \mathbf{Z}$ such that $A^{\prime}$ appears in $H^{i} \bar{K}_{D^{\prime}}^{w}$. Since $H^{i} \bar{K}_{D^{\prime}}^{w}$ is semisimple, $A^{\prime}$ is a direct summand of $H^{i} \bar{K}_{D^{\prime \prime}}^{w}$. Using (c) we see that $\operatorname{ind}_{D^{\prime}}^{D}\left(A^{\prime}\right)$ is a direct summand of $H^{i+2 \operatorname{dim} U_{P}} \bar{K}_{D}^{w}$. Hence (a) holds. 
From (a) we see that $A^{\prime} \mapsto \operatorname{ind}_{D^{\prime}}^{D}\left(A^{\prime}\right)$ (with $A^{\prime} \in \hat{D}^{\prime \text { un }}$ ) defines a group homomorphism $\mathcal{K}^{u n}\left(D^{\prime}\right) \rightarrow \mathcal{K}^{u n}(D)$ and a $\mathbf{Q}$-linear map $\mathcal{K}_{\mathbf{Q}}^{u n}\left(D^{\prime}\right) \rightarrow \mathcal{K}_{\mathbf{Q}}^{u n}(D)$ denoted again by $\operatorname{ind}_{D^{\prime}}^{D}$.

Applying this homomorphism to both sides of 44.6(a) for $D^{\prime}$ instead of $D$ and for $x \in \mathbf{W}_{I}$ and using (c) we obtain

$$
g r_{1}\left(\bar{K}_{D}^{x}\right)=\sum_{y \in \mathbf{W}_{I} ; y \leq x} P_{y, x}(1) \operatorname{ind}_{D^{\prime}}^{D}\left(g r_{1}\left(K_{D^{\prime}}^{y}\right)\right) .
$$

Here, $P_{y, x}$ are as in 43.2 for $\mathbf{W}_{I}$ or equivalently for $\mathbf{W}$. The left-hand side can be evaluated using $44.3(\mathrm{~d})$ for $D$; we obtain:

$$
\sum_{y \in \mathbf{W}_{I} ; y \leq x} P_{y, x}(1) g r_{1}\left(K_{D}^{y}\right)=\sum_{y \in \mathbf{W}_{I} ; y \leq x} P_{y, x}(1) \operatorname{ind}_{D^{\prime}}^{D}\left(g r_{1}\left(K_{D^{\prime}}^{y}\right)\right) .
$$

Since the matrix $\left(P_{y, x}\right)_{x, y \in \mathbf{W}_{I}}$ is invertible, we deduce for any $y \in \mathbf{W}_{I}$ :

$$
\operatorname{ind}_{D^{\prime}}^{D}\left(g r_{1}\left(K_{D^{\prime}}^{y}\right)\right)=g r_{1}\left(K_{D}^{y}\right) \text {. }
$$

44.11. We preserve the setup of 44.10 . Let $\Gamma, \tilde{\mathbf{W}}$ be as in 43.1 and let $\tilde{\mathbf{W}}_{I}$ be the subgroup of $\tilde{\mathbf{W}}$ generated by $\mathbf{W}_{I}$ and $\Gamma$; now $\tilde{\mathbf{W}}_{I}$ plays the same role for $\mathbf{W}_{I}$ as $\tilde{\mathbf{W}}$ for $\mathbf{W}$. For any $E^{\prime} \in \operatorname{Mod}\left(\tilde{\mathbf{W}}_{I}\right)$, the element $R_{E^{\prime}} \in \mathcal{K}_{\mathbf{Q}}^{u n}\left(D^{\prime}\right)$ is defined as in 44.6(b). Let $\operatorname{ind}_{\tilde{\mathbf{W}}_{I}}^{\tilde{\mathbf{W}}} E^{\prime} \in \operatorname{Mod}(\tilde{\mathbf{W}})$ be the induced module. We show:

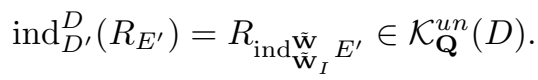

Applying $\operatorname{ind}_{D^{\prime}}^{D}$ to $44.6(\mathrm{~b})$ with $E, D$ replaced by $E^{\prime}, D^{\prime}$ and using $44.10(\mathrm{~d})$ we obtain

$$
\operatorname{ind}_{D^{\prime}}^{D}\left(R_{E^{\prime}}\right)=\left|\mathbf{W}_{I}\right|^{-1} \sum_{i \in \mathbf{Z}} \sum_{x \in \mathbf{W}_{I}}(-1)^{i+\operatorname{dim} G^{\prime}} \operatorname{tr}\left(x \varpi, E^{\prime}\right) H^{i}\left(K_{D}^{x}\right) .
$$

Using the definitions and 44.7(n) we have

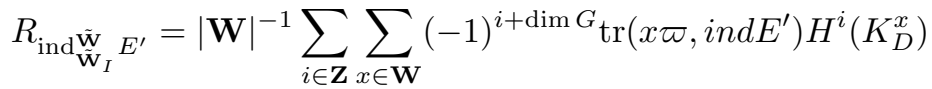

$$
\begin{aligned}
& =|\mathbf{W}|^{-1}\left|\mathbf{W}_{I}\right|^{-1} \sum_{i \in \mathbf{Z}} \sum_{x \in \mathbf{W}, y \in \mathbf{W} ; y x \varpi y^{-1} \in \mathbf{W}_{I} \varpi}(-1)^{i+\operatorname{dim} G} \operatorname{tr}\left(y x \varpi y^{-1}, E^{\prime}\right) H^{i}\left(K_{D}^{x}\right) \\
& =|\mathbf{W}|^{-1}\left|\mathbf{W}_{I}\right|^{-1} \sum_{i \in \mathbf{Z}} \sum_{z \in \mathbf{W}_{I}, y \in \mathbf{W}}(-1)^{i+\operatorname{dim} G} \operatorname{tr}\left(z \varpi, E^{\prime}\right) H^{i}\left(K_{D}^{y^{-1} z \varpi y \varpi}{ }^{-1}\right)
\end{aligned}
$$

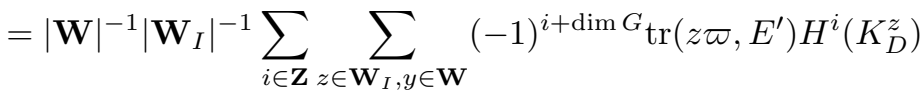

$$
\begin{aligned}
& =\left|\mathbf{W}_{I}\right|^{-1} \sum_{i \in \mathbf{Z}} \sum_{z \in \mathbf{W}_{I}}(-1)^{i+\operatorname{dim} G_{\operatorname{tr}}} \operatorname{tr}\left(z \varpi, E^{\prime}\right) H^{i}\left(K_{D}^{z}\right) .
\end{aligned}
$$

Now (a) follows since $\operatorname{dim} G=\operatorname{dim} G^{\prime} \bmod 2$.

44.12. We preserve the setup of 44.10. Let $\mathbf{s}$ be a sequence in $\mathbf{I}$. From 29.14 we see that $\operatorname{res}_{D}^{D^{\prime}}\left(\bar{K}_{D}^{\mathbf{s}}\right) \cong \bigoplus_{\mathbf{t} \in \mathcal{T}} \bar{K}_{D^{\prime}}^{\mathbf{t}}\left[-d_{\mathbf{t}}\right]$ where $\mathcal{T}$ is a certain finite collection of sequences in $I$ and $d_{\mathbf{t}}$ are integers. Since $\bar{K}_{D}^{\mathbf{s}} \cong \bigoplus_{i} H^{i}\left(\bar{K}_{D}^{\mathbf{s}}\right)[-i], \bar{K}_{D^{\prime}}^{\mathbf{t}} \cong \bigoplus_{i} H^{i}\left(\bar{K}_{D^{\prime}}^{\mathbf{t}}\right)[-i]$, we have

$$
\bigoplus_{i} \operatorname{res}_{D}^{D^{\prime}}\left(H^{i}\left(\bar{K}_{D}^{\mathbf{s}}\right)\right)[-i] \cong \bigoplus_{\mathbf{t} \in \mathcal{T}, i} H^{i}\left(\bar{K}_{D^{\prime}}^{\mathbf{t}}\right)\left[-i-d_{\mathbf{t}}\right] .
$$


By $31.14, \operatorname{res}_{D}^{D^{\prime}}\left(H^{i}\left(\bar{K}_{D}^{\mathbf{s}}\right)\right)$ is a perverse sheaf on $D^{\prime}$. Hence taking $H^{i}$ for both sides of (a) we obtain

$$
\operatorname{res}_{D}^{D^{\prime}}\left(H^{i}\left(\bar{K}_{D}^{\mathbf{s}}\right)\right) \cong \bigoplus_{\mathbf{t} \in \mathcal{T}} H^{i-d_{\mathbf{t}}}\left(\bar{K}_{D^{\prime}}^{\mathbf{t}}\right)
$$

In particular, if $A \in \hat{D}^{u n}$, then $\operatorname{res}_{D}^{D^{\prime}}(A)$ is a direct sum of objects in $\hat{D}^{\text {un }}$. Hence $A \mapsto \operatorname{res}_{D}^{D^{\prime}}(A)$ (with $A \in \hat{D}^{u n}$ ) defines a group homomorphism $\mathcal{K}^{\text {un }}(D) \rightarrow \mathcal{K}^{u n}\left(D^{\prime}\right)$ and a $\mathbf{Q}$-linear map $\mathcal{K}^{u n}(D)_{\mathbf{Q}} \rightarrow \mathcal{K}_{\mathbf{Q}}^{u n}\left(D^{\prime}\right)$ denoted again by $\operatorname{res}_{D}^{D^{\prime}}$. Taking the alternating sum over $i$ in (b) we obtain:

$$
\operatorname{res}_{D}^{D^{\prime}}\left(g r_{1}\left(\bar{K}_{D}^{\mathbf{s}}\right)\right)=\sum_{\mathbf{t} \in \mathcal{T}}(-1)^{d_{\mathbf{t}}} g r_{1}\left(\bar{K}_{D^{\prime}}^{\mathbf{t}}\right)
$$

For any $\xi \in \mathcal{K}_{\mathbf{Q}}^{u n}(D), \xi^{\prime} \in \mathcal{K}_{\mathbf{Q}}^{u n}\left(D^{\prime}\right)$ we have

$$
\left(\operatorname{res}_{D}^{D^{\prime}}(\xi): \xi^{\prime}\right)=\left(\xi: \operatorname{ind}_{D^{\prime}}^{D}\left(\xi^{\prime}\right)\right)
$$

where the first (:) refers to $D^{\prime}$ and the second (:) refers to $D$. Indeed, we can assume that $\xi=A \in \hat{D}^{u n}, \xi^{\prime}=A^{\prime} \in \hat{D}^{\prime u n}$; in this case (d) follows from the equalities in 30.9 and the semisimplicity of the perverse sheaves $\operatorname{res}_{D}^{D^{\prime}}(A), \operatorname{ind}_{D^{\prime}}^{D}\left(A^{\prime}\right)$.

The following subspaces of $\mathcal{K}_{\mathbf{Q}}^{u n}(D)$ coincide:

-the subspace (1) spanned by the $R_{E}$ (with $\left.E \in \operatorname{Mod}(\tilde{\mathbf{W}})\right)$;

-the subspace (2) spanned by the $R_{E}$ (with $E \in \operatorname{Irr}(\tilde{\mathbf{W}})$ );

-the subspace (3) spanned by the elements $g r_{1}\left(K_{D}^{x}\right)$ (with $x \in \mathbf{W}$ );

-the subspace (4) spanned by the elements $g r_{1}\left(K_{D}^{\mathbf{s}}\right)$ for various sequences $\mathbf{s}$ in $\mathbf{I}$. Indeed, (1) $\subset$ (3) by 44.6(b); (3) $\subset$ (2) by 44.7(i); (2) $\subset$ (1) obviously; moreover, $(3)=(4)$ by the arguments in 31.7 . We denote any of the four subspaces above by $V_{D}$. We define similarly a subspace $V_{D^{\prime}}$ of $\mathcal{K}_{\mathbf{Q}}^{\text {un }}\left(D^{\prime}\right)$. We show

$$
\operatorname{res}_{D}^{D^{\prime}}\left(R_{E}\right)=R_{\left.E\right|_{\tilde{\mathbf{w}}_{I}}}
$$

where $\left.E\right|_{\tilde{\mathbf{W}}_{I}} \in \operatorname{Mod}\left(\tilde{\mathbf{W}}_{I}\right)$ is the restriction of $E$. From (c) we see that $\operatorname{res}_{D}^{D^{\prime}} \operatorname{maps}$ $V_{D}$ into $V_{D^{\prime}}$. Thus both sides of (e) are in $V_{D^{\prime}}$. Now the restriction of (:) (for $D^{\prime}$ ) to $V_{D^{\prime}}$ is nondegenerate (we use the analogue of $44.7(\mathrm{l})$ for $D^{\prime}$ ). Hence to prove (e) it is enough to show that

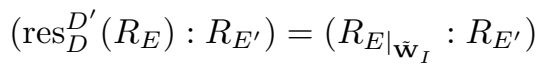

for any $E^{\prime} \in \operatorname{Mod}\left(\tilde{\mathbf{W}}_{I}\right)$. By (d) and 44.11(a), the left-hand side of (f) is equal to

$$
\left(R_{E}: \operatorname{ind}_{D^{\prime}}^{D}\left(R_{E^{\prime}}\right)=\left(R_{E}: R_{\operatorname{ind}_{\tilde{\mathbf{w}}_{I}}^{\tilde{\tilde{W}}} E^{\prime}}\right) .\right.
$$

Using $44.7(1)$ for $D$ and for $D^{\prime}$ we see that it is enough to use the equality

$$
|\mathbf{W}|^{-1} \sum_{x \in \mathbf{W}} \operatorname{tr}(x \varpi, E) \operatorname{tr}\left(x \varpi, \operatorname{ind}_{\tilde{\mathbf{W}}_{I}}^{\tilde{\mathbf{W}}} E^{\prime}\right)=\left|\mathbf{W}_{I}\right|^{-1} \sum_{x \in \mathbf{W}_{I}} \operatorname{tr}(x \varpi, E) \operatorname{tr}\left(x \varpi, E^{\prime}\right)
$$

which follows from the standard character formula for an induced representation. This proves (f) and hence (e). 
44.13. Let $x \in \mathbf{W}$ be such that for any $y \in \mathbf{W}$ we have $y x \varpi y^{-1} \varpi^{-1} \notin \mathbf{W}_{I}$. We show:

$$
\operatorname{res}_{D}^{D^{\prime}}\left(g r_{1}\left(K_{D}^{x}\right)\right)=0 .
$$

Using 44.7(i), we see that it is enough to show:

$$
(-1)^{\operatorname{dim} G} \sum_{E \in \mathfrak{E}} \operatorname{tr}(x \varpi, E) \operatorname{res}_{D}^{D^{\prime}}\left(R_{E}\right)=0 .
$$

Using 44.12(e) and 44.6(b) for $D^{\prime}$, we see that left-hand side is

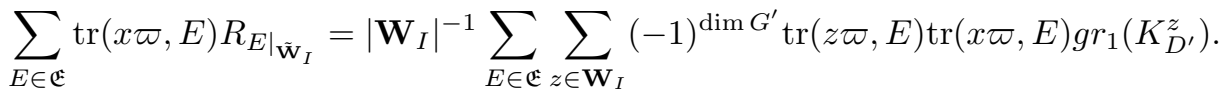

To show that this is zero it is enough to show that for any $z \in \mathbf{W}_{I}$ we have

$$
\sum_{E \in \mathfrak{E}} \operatorname{tr}(z \varpi, E) \operatorname{tr}(x \varpi, E)=0 .
$$

The left-hand side is equal to $|\tilde{\mathbf{W}}|^{-1}|\mathbf{W}|$ times $\sum_{E} \operatorname{tr}(z \varpi, E) \operatorname{tr}\left((x \varpi)^{-1}, E\right)$ where $E$ runs over the simple $\overline{\mathbf{Q}}_{l}[\tilde{\mathbf{W}}]$-modules up to isomorphism. (A module $E$ whose restriction to $\mathbf{W}$ is not simple contributes 0 to the last sum.) It is enough to show that the last sum is 0 . It is also enough to show that $z \varpi$ and $x \varpi$ are not conjugate in $\tilde{\mathbf{W}}$. But this follows from our assumption on $x$. This proves (a).

44.14. An element $w \in \mathbf{W}$ is said to be $D$-anisotropic if the following condition holds: for any $x \in \mathbf{W}, I \varsubsetneqq \mathbf{I}$ such that $\epsilon(I)=I$ we have $x w \epsilon(x)^{-1} \notin \mathbf{W}_{I}$. Let $A \in \hat{D}^{u n}$.

We show:

(a) $A$ is cuspidal if and only if any $w \in \mathbf{W}$ such that $\left(A: g r_{1}\left(K_{D}^{w}\right)\right) \neq 0$ is $D$-anisotropic.

Assume first that $A$ is not cuspidal. By 31.15 there exists $I \varsubsetneqq \mathbf{I}, \epsilon(I)=I$ and $P \in$ $\mathcal{P}_{I}$ (so that $N_{D} P \neq \emptyset$ ) such that setting $D^{\prime}=N_{D} P / U_{P}, G^{\prime}=N_{G} P / U_{P}$ we have $\operatorname{res}_{D}^{D^{\prime}}(A) \neq 0$. By 31.14 and 44.12 , $\operatorname{res}_{D}^{D^{\prime}}(A)$ is an $\mathbf{N}$-linear combination of objects in $\hat{D}^{\prime \text { un }}$. Hence there exists $x \in \mathbf{W}_{I}$ and $i \in \mathbf{Z}$ such that $\left(\operatorname{res}_{D}^{D^{\prime}}(A): H^{i}\left(\bar{K}_{D}^{x}\right)\right) \neq 0$. Using $44.7(\mathrm{~m})$ for $D^{\prime}$ we see that there exists $E^{\prime} \in \operatorname{Irr}\left(\tilde{\mathbf{W}}_{I}\right)$ such that $\left(\operatorname{res}_{D}^{D^{\prime}}(A)\right.$ : $\left.R_{E^{\prime}}\right) \neq 0$. Hence there exists $y \in \mathbf{W}_{I}$ such that $\left(\operatorname{res}_{D}^{D^{\prime}}(A): g r_{1}\left(K_{D^{\prime}}^{y}\right)\right) \neq 0$. Using 44.12(d) we deduce $\left(A: \operatorname{ind}_{D^{\prime}}^{D}\left(g r_{1}\left(K_{D^{\prime}}^{y}\right)\right) \neq 0\right.$ and using $44.10(\mathrm{~d})$ we see that $\left(A: g r_{1}\left(K_{D}^{y}\right)\right) \neq 0$. Since $y \in \mathbf{W}_{I}, y$ is not $D$-anisotropic.

Conversely, assume that there exist $w \in \mathbf{W}, x \in \mathbf{W}, I \varsubsetneqq \mathbf{I}$ such that $(A$ : $\left.g r_{1}\left(K_{D}^{w}\right)\right) \neq 0, \epsilon(I)=I$ and $x w \epsilon(x)^{-1} \in \mathbf{W}_{I}$. Using 44.7(n) we see that we can assume that $x=1, w \in \mathbf{W}_{I}$. Choose $P \in \mathcal{P}_{I}$ (so that $N_{D} P \neq \emptyset$ ) and set $D^{\prime}=$ $N_{D} P / U_{P}, G^{\prime}=N_{G} P / U_{P}$. Using $44.10(\mathrm{~d})$ we see that $\left(A: \operatorname{ind}_{D^{\prime}}^{D}\left(g r_{1}\left(K_{D}^{w}\right)\right)\right) \neq 0$. Using $44.12(\mathrm{~d})$ we see that $\left(\operatorname{res}_{D}^{D^{\prime}}(A): g r_{1}\left(K_{D^{\prime}}^{w}\right)\right) \neq 0$ so that $\operatorname{res}_{D}^{D^{\prime}}(A) \neq 0$. Thus $A$ is not cuspidal. This proves (a).

We show:

(b) Let $w \in \mathbf{W}$ be such that $w$ is D-anisotropic. Then $l(w)=\left|\mathbf{I}_{\epsilon}\right| \bmod 2$ where $\mathbf{I}_{\epsilon}$ is the set of orbits of $\epsilon: \mathbf{I} \rightarrow \mathbf{I}$.

We use the notation in 42.7. We consider the equality

$$
(-1)^{|\mathbf{I}|} H_{c}^{|\mathbf{I}|}\left(\mathcal{V}_{\mathbf{R}}\right)=\sum_{\eta}(-1)^{r_{\eta}} H^{r_{\eta}}\left(\mathcal{V}_{\mathbf{R}}^{\eta}\right)
$$


(see 42.7) in the Grothendieck group of $\mathbf{W}^{D}$-modules. Taking the trace of $w \underline{D} \in$ $\mathbf{W}^{D}$ we obtain

$$
(-1)^{|\mathbf{I}|} \operatorname{det}\left(w \underline{D}, \mathcal{V}_{\mathbf{R}}\right)=\sum_{\eta} t_{\eta}
$$

where

$$
t_{\eta}=(-1)^{r_{\eta}} \operatorname{tr}\left(w \underline{D}, \bigoplus_{J \in \eta} \bigoplus_{F \in \mathcal{F}_{J}} \Lambda^{r_{\eta}}(|F|) .\right.
$$

Since $w \underline{D}$ permutes the summands in the last direct sum, we have $t_{\eta}=0$ unless there exist $J \in \eta$ and $F \in \mathcal{F}_{J}$ such that $\underline{D}(J)=J$ and $w \underline{D}(F)=F$. For such $J, F$ we can find $F_{J} \in \mathcal{F}_{J}$ such that $\underline{D}\left(F_{J}\right)=F_{J}$ and $\left\{y \in \mathbf{W} ; y\left(F_{J}\right)=F_{J}\right\}=\mathbf{W}_{J}$; moreover, $F=x^{-1}\left(F_{J}\right)$ for some $x \in \mathbf{W}$ and $w \epsilon(x)^{-1}\left(F_{J}\right)=x^{-1}\left(F_{J}\right)$ so that $x w \epsilon(x)^{-1}\left(F_{J}\right)=F_{J}$ and $x w \epsilon(x)^{-1} \in \mathbf{W}_{J}$. Since $w$ is $D$-anisotropic, we see that $J=\mathbf{I}$. Thus $t_{\eta}=0$ unless $\eta=\{\mathbf{I}\}$. On the other hand, if $\eta=\{\mathbf{I}\}$, then $\mathcal{F}_{J}=\{0\}, r_{\eta}=0$ and $t_{\eta}=1$. Thus we have $(-1)^{|\mathbf{I}|} \operatorname{det}\left(w \underline{D}, \mathcal{V}_{\mathbf{R}}\right)=1$. Note that $\operatorname{det}\left(w, \mathcal{V}_{\mathbf{R}}\right)=(-1)^{l(w)}$. Since $\underline{D}$ permutes a basis of $\mathcal{V}_{\mathbf{R}}$ indexed by $\mathbf{I}$ (according to $\epsilon$ ) we have $\operatorname{det}\left(\underline{D}, \mathcal{V}_{\mathbf{R}}\right)=(-1)^{|\mathbf{I}|-\left|\mathbf{I}_{\epsilon}\right|}$. We see that $(-1)^{l(w)}(-1)^{\left|\mathbf{I}_{\epsilon}\right|}=1$. This proves (b).

44.15. Let $P$ be a parabolic subgroup of $G^{0}$ such that $N_{D} P \neq \emptyset$. Let $D^{\prime}=$ $N_{D} P / U_{P}$ (a connected component of $\left.N_{G} P / U_{P}\right)$. We show:

(a) If $A^{\prime} \in \hat{D}^{\prime \text { un }}, A \in \hat{D}^{u n}$, are such that $A$ appears with nonzero coefficient in $\operatorname{ind}_{D^{\prime}}^{D}\left(A^{\prime}\right)$ (or equivalently $A^{\prime}$ appears with nonzero coefficient in $\operatorname{res}_{D}^{D^{\prime}}(A)$ ), then $\mathbf{e}^{A}=\mathbf{e}^{A^{\prime}}$. Moreover, $\operatorname{codim}(\operatorname{supp}(A))=\operatorname{codim}\left(\operatorname{supp}\left(A^{\prime}\right)\right) \bmod 2$.

We can find $I \subset \mathbf{I}, \epsilon(I)=I$ such that $P \in \mathcal{P}_{I}$ and $w \in \mathbf{W}_{I}, i \in \mathbf{Z}$ such that $A^{\prime}$ is a direct summand of $H^{i}\left(\bar{K}_{D^{\prime}}^{w}\right)$. Then $\operatorname{ind}_{D^{\prime}}^{D}\left(A^{\prime}\right)$ is a direct summand of $\operatorname{ind}_{D^{\prime}}^{D}\left(H^{i} \bar{K}_{D^{\prime}}^{w}\right)$, hence a direct summand of $H^{i+2 \operatorname{dim} U_{P}} \bar{K}_{D}^{w}$ (see $44.10(\mathrm{c})$ ). It follows that $A$ is a direct summand of $H^{i+2 \operatorname{dim} U_{P}} \bar{K}_{D}^{w}$. By definition we have $\mathbf{e}^{A^{\prime}}=(-1)^{i+\operatorname{dim}\left(P / U_{P}\right)}, \mathbf{e}^{A}=(-1)^{i+2 \operatorname{dim} U_{P}+\operatorname{dim} G^{0}}$. Thus, $\mathbf{e}^{A}=\mathbf{e}^{A^{\prime}}$. This proves the first statement of (a). We can find a parabolic subgroup $P_{1}$ of $G^{0}$ such that $N_{D} P_{1} \neq \emptyset, P_{1} \subset P$ and $A_{1} \in \hat{D}_{1}^{\text {unc }}$ (where $D_{1}=N_{D} P_{1} / U_{P_{1}}$ ) such that $A^{\prime}$ is a component of $\operatorname{ind}_{D_{1}}^{D^{\prime}}\left(A_{1}\right)$, hence $A$ is a component of $\operatorname{ind}_{D_{1}}^{D}\left(A_{1}\right)$. To prove the second statement of (a) it is enough to show that $(-1)^{\operatorname{codim}(\operatorname{supp}(A))}=(-1)^{\operatorname{codim}\left(\operatorname{supp}\left(A_{1}\right)\right)}$, $(-1)^{\operatorname{codim}\left(\operatorname{supp}\left(A^{\prime}\right)\right)}=(-1)^{\operatorname{codim}\left(\operatorname{supp}\left(A_{1}\right)\right)}$. Thus we are reduced to the case where $A^{\prime}$ is cuspidal. In this case, by 3.13(b) we have $\operatorname{dim} \operatorname{supp}(A)=\operatorname{dim}\left(G^{0}\right)-\operatorname{dim}\left(P / U_{P}\right)+$ $\operatorname{dim} \operatorname{supp}\left(A^{\prime}\right)$. Thus, $\operatorname{codim}(\operatorname{supp}(A))=\operatorname{codim}\left(\operatorname{supp}\left(A^{\prime}\right)\right)$ and $($ a) is proved.

We show:

(b) If $A \in \hat{D}^{u n}$ and $A^{\circ} \in \hat{D}^{u n}$ is defined by $\mathbf{d}(A)=(-1)^{\operatorname{codim}(\operatorname{supp}(A))} A^{\circ}$ (see 44.8(a)), then $\mathbf{e}^{A^{\circ}}=\mathbf{e}^{A}$.

If $P, D^{\prime}$ are as in (a), then, by (a), $\operatorname{ind}_{D^{\prime}}^{D} \operatorname{res}_{D}^{D^{\prime}}(A)$ is a linear combination of objects $A_{1} \in \hat{D}^{u n}$ with $\mathbf{e}^{A_{1}}=\mathbf{e}^{A}$. Since $\mathbf{d}(A)$ is an alternating sum of elements of the form $\operatorname{ind}_{D^{\prime}}^{D} \operatorname{res}_{D}^{D^{\prime}}(A)$, we see that $\mathbf{d}(A)$ is a linear combination of objects $A_{1} \in \hat{D}^{u n}$ with $\mathbf{e}^{A_{1}}=\mathbf{e}^{A}$. Now (b) follows.

Let $x \in \mathbf{W}$. We show:

(c) The element $R_{\aleph_{x \varpi}} \in \mathcal{K}_{\mathbf{Q}}^{u n}(D)$ is a $\mathbf{Z}$-linear combination of objects $A \in \hat{D}^{\text {un }}$ such that $\mathbf{e}^{A}=(-1)^{l(x)-\mathbf{a}(x)}$. 
Let $\mathbf{c}$ be the two-sided cell containing $x$. Using $43.12(\mathrm{~b})$, for any $A \in \hat{D}^{u n}$ we have (with notation in 43.12):

$$
\begin{aligned}
& \left(A: R_{\aleph_{x \varpi}}\right)=\sum_{E \in \underline{\operatorname{Ir}}(\tilde{\mathbf{W}})} \frac{1}{2}\left(\operatorname{tr}\left(c_{x \varpi}^{\dagger}, E^{v} ;-\mathbf{a}(x)\right)\right. \\
& -\sum_{y, j ; y \prec x, j>0}(-1)^{-l(x)+l(y)} a_{y, x ; j} \operatorname{tr}\left(c_{y \varpi}^{\dagger}, E^{v} ;-\mathbf{a}(x)-j\right)\left(A: R_{E}\right) .
\end{aligned}
$$

From 44.7(j) we have for any $A \in \hat{D}^{u n}$ and $z \in \mathbf{W}$ :

$$
\begin{aligned}
& \sum_{j \in \mathbf{Z}}\left(\mathbf{d}(A): H^{j}\left(\bar{K}_{D}^{z}\right)\right)(-v)^{j} \\
& =(-1)^{\operatorname{dim} G} v^{\operatorname{dim} G+l(z)} \sum_{E \in \underline{\operatorname{Irr}}(\tilde{\mathbf{W}})} \frac{1}{2}\left(\mathbf{d}(A): R_{E}\right) \operatorname{tr}\left(c_{z \varpi}, E^{v}\right) \\
& =(-1)^{\operatorname{dim} G} v^{\operatorname{dim} G+l(z)} \sum_{E \in \underline{\operatorname{Irr}}(\tilde{\mathbf{W}})} \frac{1}{2}\left(A: R_{E \otimes \operatorname{sgn}}\right) \operatorname{tr}\left(c_{z \varpi}, E^{v}\right) \\
& =(-1)^{\operatorname{dim} G} v^{\operatorname{dim} G+l(z)} \sum_{E \in \underline{\operatorname{Ir}}_{(\tilde{\mathbf{W}})}} \frac{1}{2}\left(A: R_{E}\right) \operatorname{tr}\left(c_{z \varpi},(E \otimes \operatorname{sgn})^{v}\right) \\
& =(-1)^{\operatorname{dim} G} v^{\operatorname{dim} G+l(z)} \sum_{E \in \underline{\operatorname{Irr}}(\tilde{\mathbf{W}})} \frac{1}{2}\left(A: R_{E}\right) \operatorname{tr}\left(c_{z \varpi}^{\dagger}, E^{v}\right) .
\end{aligned}
$$

(We have used 44.8(c), 43.4(c).) Hence for any $N \in \mathbf{Z}$ we have

$$
\sum_{E \in \underline{\operatorname{Irr}}(\tilde{\mathbf{W}})} \frac{1}{2}\left(A: R_{E}\right) \operatorname{tr}\left(c_{z \varpi}^{\dagger}, E^{v} ; N\right)=\left(\mathbf{d}(A): H^{N+\operatorname{dim} G+l(z)}\left(\bar{K}_{D}^{z}\right)\right)(-1)^{N+l(z)} .
$$

Introducing this in (c) we obtain

$$
\begin{aligned}
& \left(A: R_{\aleph_{x \varpi \varpi}}\right)=(-1)^{l(x)-\mathbf{a}(x)}\left(\mathbf{d}(A): H^{\operatorname{dim} G+l(x)-\mathbf{a}(x)}\left(\bar{K}_{D}^{x}\right)\right) \\
& -\sum_{y, j ; y \prec x, j>0} a_{y, x ; j}(-1)^{l(x)-\mathbf{a}(x)-j}\left(\mathbf{d}(A): H^{\operatorname{dim} G+l(y)-\mathbf{a}(x)-j}\left(\bar{K}_{D}^{y}\right)\right) .
\end{aligned}
$$

Since $a_{y, x ; j}$ are integers (see 43.12$)$ we see that $\left(A: R_{\aleph_{x \varpi}}\right) \in \mathbf{Z}$. Assume now that $\left(A: R_{\aleph_{x \varpi}}\right) \neq 0$. Using (e) and 43.12 we see that either

$$
\left(A^{\circ}: H^{\operatorname{dim} G+l(x)-\mathbf{a}(x)}\left(\bar{K}_{D}^{x}\right)\right) \neq 0
$$

or there exist $y, j$ such that $j=l(x)+l(y) \bmod 2$,

$$
\left(A^{\circ}: H^{\operatorname{dim} G+l(y)-\mathbf{a}(x)-j}\left(\bar{K}_{D}^{y}\right)\right) \neq 0
$$

(here $A^{\circ}$ is as in 44.8(a)). In the first case we have $\mathbf{e}^{A^{\circ}}=(-1)^{l(x)-\mathbf{a}(x)}$. In the second case we have $\mathbf{e}^{A^{\circ}}=(-1)^{l(y)-\mathbf{a}(x)-j}=(-1)^{l(x)-\mathbf{a}(x)}$ since $j=l(x)+l(y)$ mod 2. This implies (c) in view of (b).

Note that $D$ has property $\tilde{\mathfrak{A}}$ (see 44.7 ) if and only if for any $A \in \hat{D}^{u n}$ we have $\mathbf{e}^{A}=(-1)^{\operatorname{codim}(\operatorname{supp}(A))}$. 
44.16. We show that if $D$ has property $\tilde{\mathfrak{A}}$, then for any $A \in \hat{D}^{u n}, w \in \mathbf{W}, i \in \mathbf{Z}$ we have

$$
(-1)^{i+\operatorname{dim} G}\left(A: \mathbf{d}\left(H^{i}\left(\bar{K}_{D}^{w}\right)\right)\right) \in \mathbf{N} .
$$

Indeed, the expression (a) is equal to $(-1)^{i+\operatorname{dim} G}\left(\mathbf{d}(A): H^{i}\left(\bar{K}_{D}^{w}\right)\right)$ (see 38.10(e)). If this is $\neq 0$, then it is equal to $(-1)^{\operatorname{codim}(\operatorname{supp}(A))} \mathbf{e}^{A^{\circ}}\left(A^{\circ}: H^{i}\left(\bar{K}_{D}^{w}\right)\right)$. By property $\tilde{\mathfrak{A}}$ for $A^{\circ}$ and $44.8(\mathrm{e})$, this is equal to

$$
(-1)^{\operatorname{codim}(\operatorname{supp}(A))}(-1)^{\operatorname{codim}\left(\operatorname{supp}\left(A^{\circ}\right)\right)}\left(A^{\circ}: H^{i}\left(\bar{K}_{D}^{w}\right)\right)=\left(A^{\circ}: H^{i}\left(\bar{K}_{D}^{w}\right)\right) \in \mathbf{N} .
$$

This proves (a).

44.17. Let $x \in \mathbf{W}$ and let $\mathbf{c}$ be the two-sided cell of $\mathbf{W}$ that contains $x$. Let $a$ be the value of $\mathbf{a}: \mathbf{W} \rightarrow \mathbf{N}$ on $\mathbf{c}$. We show that in $\mathcal{K}_{\mathbf{Q}}^{u n}(D)$ we have:

(a) $(-1)^{-a+l(x)} H^{-a+l(x)+\operatorname{dim} G}\left(\bar{K}_{D}^{x}\right)$

$$
=R_{\aleph_{x \varpi} \otimes \mathrm{sgn}}+\text { Q-linear combination of elements } R_{\aleph_{x^{\prime} \varpi} \otimes \operatorname{sgn}} \text { with } x^{\prime} \prec x,
$$

(b) $(-1)^{-a+l(x)} \mathbf{d}\left(H^{-a+l(x)+\operatorname{dim} G}\left(\bar{K}_{D}^{x}\right)\right)$

$$
=R_{\aleph_{x \varpi}}+\text { Q-linear combination of elements } R_{\aleph_{x^{\prime} \varpi}} \text { with } x^{\prime} \prec x \text {. }
$$

By $44.7(\mathrm{~m})$, the left-hand side of (b) is equal to $\sum_{E} \frac{1}{2} \operatorname{tr}\left(c_{x} \tilde{T}_{\varpi}, E^{v} ;-a\right) \mathbf{d}\left(R_{E}\right)$. By 44.8(c) and 43.4(b), 43.6(b), this equals

$$
\begin{aligned}
& \sum_{E} \frac{1}{2} \operatorname{tr}\left(c_{x} \tilde{T}_{\varpi}, E^{v} ;-a\right) R_{E \otimes \operatorname{sgn}}=\sum_{E} \frac{1}{2} \operatorname{tr}\left(c_{x} \tilde{T}_{\varpi},(E \otimes \operatorname{sgn})^{v} ;-a\right) R_{E} \\
& =\sum_{E} \frac{1}{2} \operatorname{tr}\left(c_{x \varpi}^{\dagger}, E^{v} ;-a\right) R_{E}=\sum_{E ; \mathbf{c}_{E} \preceq \mathbf{c}} \frac{1}{2} \operatorname{tr}\left(c_{x \varpi}^{\dagger}, E^{v} ;-a\right) R_{E}=b^{\prime}+b^{\prime \prime}
\end{aligned}
$$

where

$$
\begin{aligned}
b^{\prime} & =\sum_{E ; \mathbf{c}_{E}=\mathbf{c}} \frac{1}{2} \operatorname{tr}\left(c_{x \varpi}^{\dagger}, E^{v} ;-a\right) R_{E}=\sum_{E ; \mathbf{c}_{E}=\mathbf{c}} \frac{1}{2} \operatorname{tr}\left(t_{x} \varpi, E^{\infty}\right) R_{E} \\
& =\sum_{E} \frac{1}{2} \operatorname{tr}\left(t_{x} \varpi, E^{\infty}\right) R_{E}=R_{\aleph_{x \varpi}}, \\
b^{\prime \prime} & =\sum_{E ; \mathbf{c}_{E} \prec \mathbf{c}} \frac{1}{2} \operatorname{tr}\left(c_{x \varpi}^{\dagger}, E^{v} ;-a\right) R_{E} .
\end{aligned}
$$

Now $b^{\prime \prime}$ is a Z-linear combination of elements of the form $R_{E}$ where $E$ is such that $\mathbf{c}_{E} \prec \mathbf{c}$ and these elements are $\mathbf{Q}$-linear combinations of elements of the form $R_{\aleph_{x^{\prime} \varpi}}$ for various $x^{\prime} \in \mathbf{W}$ such that $x^{\prime} \prec x$, by $43.10(\mathrm{~b})$. This proves (b). Now (a) is obtained by applying $\mathbf{d}$ to both sides of (b) and using the equality $\mathbf{d}\left(R_{\phi}\right)=R_{\phi \otimes \text { sgn }}$ for any $\phi \in \mathcal{R}(\tilde{\mathbf{W}})$ (see $44.8(\mathrm{c})$ ).

Now let $a^{\prime}$ be the value of $\mathbf{a}: \mathbf{W} \rightarrow \mathbf{N}$ on the two-sided cell $w_{0} \mathbf{c}=\mathbf{c} w_{0}$. We show:

$$
(-1)^{-a^{\prime}+l\left(w_{0} x\right)} H^{-a^{\prime}+l\left(w_{0} x\right)+\operatorname{dim} G}\left(\bar{K}_{D}^{w_{0} x}\right)=R_{\aleph_{w_{0} x \varpi} \otimes \operatorname{sgn}}
$$

$$
+ \text { Q-linear combination of elements } R_{\aleph_{w_{0} x^{\prime} \varpi} \otimes \operatorname{sgn}} \text { with } x \prec x^{\prime} \text {. }
$$

This is obtained by replacing $x$ by $w_{0} x$ in (a) and noting that for $y \in \mathbf{W}$ we have $w_{0} y \prec w_{0} x$ if and only if $x \prec y$. 
In the remainder of this section we assume that $D$ satisfies property $\tilde{\mathfrak{A}}$ (in addition to property $\mathfrak{A})$.

For any $x \in \mathbf{W}$ we set $r_{x}=R_{\aleph_{x \varpi}}, \tilde{r}_{x}=(-1)^{-\mathbf{a}\left(w_{0} x\right)+l\left(w_{0} x\right)} R_{\aleph_{w_{0} x \varpi} \otimes \mathrm{sgn}}$. We note the following properties of the elements $r_{x}, \tilde{r}_{x}$ :

(i) $\left(r_{x}: r_{x^{\prime}}\right)=0$ whenever $x \not x^{\prime}$;

(ii) for any two-sided cell $\mathbf{c}$, the $\mathbf{Q}$-vector space spanned by $\left\{r_{x} ; x \in \mathbf{c}\right\}$ coincides with the $\mathbf{Q}$-vector space spanned by $\left\{\tilde{r}_{x} ; x \in \mathbf{c}\right\}$;

(iii) for any $x \in \mathbf{W}$ there exist $d_{x, x^{\prime}} \in \mathbf{Q}$ defined for $x^{\prime} \prec x$ such that $\left(A: r_{x}+\sum_{x^{\prime} ; x^{\prime} \prec x} d_{x, x^{\prime}} r_{x^{\prime}}\right) \in \mathbf{N}$ for any $A \in \hat{D}^{u n}$

(iv) for any $x \in \mathbf{W}$ there exist $\tilde{d}_{x, x^{\prime}} \in \mathbf{Q}$ defined for $x \prec x^{\prime}$ such that $\left(A: \tilde{r}_{x}+\sum_{x^{\prime} ; x \prec x^{\prime}} \tilde{d}_{x, x^{\prime}} \tilde{r}_{x^{\prime}}\right) \in \mathbf{N}$ for any $A \in \hat{D}^{u n}$.

In the setup of (ii), let $V_{\mathbf{c}}$ be the $\mathbf{Q}$-vector space spanned by $R_{E}$ with $E \in \operatorname{Irr}(\tilde{\mathbf{W}})$ such that $\mathbf{c}_{E}=\mathbf{c}$. From the definitions, for any $x \in \mathbf{c}, r_{x}$ belongs to $V_{\mathbf{c}}$. Conversely, for any $E \in \operatorname{Irr}(\tilde{\mathbf{W}})$ such that $\mathbf{c}_{E}=\mathbf{c}, R_{E}$ belongs to the first vector space in (ii), by $43.10(\mathrm{~b})$. Thus the first vector space in (ii) is equal to $V_{\mathbf{c}}$. Let $V_{\mathbf{c}}^{\prime}$ be the $\mathbf{Q}$ vector space spanned by $R_{E^{\prime} \otimes \text { sgn }}$ with $E^{\prime} \in \operatorname{Irr}(\tilde{\mathbf{W}})$ such that $\mathbf{c}_{E^{\prime}}=w_{0} \mathbf{c}$. From the definitions, for any $x \in \mathbf{c}, \tilde{r}_{x}$ belongs to $V_{\mathbf{c}}^{\prime}$. Conversely, for any $E^{\prime} \in \operatorname{Irr}(\tilde{\mathbf{W}})$ such that $\mathbf{c}_{E^{\prime}}=w_{0} \mathbf{c}, R_{E^{\prime} \otimes \text { sgn }}$ belongs to the second vector space in (ii), by $43.10(\mathrm{~b})$. Thus the second vector space in (ii) is equal to $V_{\mathbf{c}}^{\prime}$. If $E^{\prime} \in \operatorname{Irr}(\tilde{\mathbf{W}})$, then we have $\mathbf{c}_{E^{\prime}}=w_{0} \mathbf{c}$ if and only $\mathbf{c}_{E^{\prime} \otimes \text { sgn }}=\mathbf{c}$ (a known property of two-sided cells). It follows that $V_{\mathbf{c}}=V_{\mathbf{c}}^{\prime}$ and (ii) is proved.

We prove (i). Let $\mathbf{c}, \mathbf{c}^{\prime}$ be the two-sided cells that contain $x, x^{\prime}$ respectively. Assume that $\mathbf{c} \neq \mathbf{c}^{\prime}$. It is enough to show that $\left(h: h^{\prime}\right)=0$ for any $h \in V_{\mathbf{c}}, h^{\prime} \in V_{\mathbf{c}^{\prime}}$. Hence it is enough to show that if $E, E^{\prime} \in \operatorname{Irr}(\tilde{\mathbf{W}})$ are such that $\mathbf{c}_{E}=\mathbf{c}, \mathbf{c}_{E^{\prime}}=\mathbf{c}^{\prime}$, then $\left(R_{E}: R_{E^{\prime}}\right)=0$. This follows from $44.7(\mathrm{l})$ since $E, E^{\prime}$ have nonisomorphic restrictions to $\mathbf{Q}[\mathbf{W}]$.

Now (iv) follows from (c) and (iii) follows from (b) in view of 44.16(a).

From (i)-(iv) we deduce, by a general result in [L3, 16.8(III)], that:

$$
\left(A: r_{x}\right) \in \mathbf{N}, \quad\left(A: \tilde{r}_{x}\right) \in \mathbf{N} \text { for any } A \in \hat{D}^{u n}, x \in \mathbf{W} .
$$

We show:

(e) Let $A \in \hat{D}^{u n}$ and let $E, E^{\prime} \in \operatorname{Irr}(\tilde{\mathbf{W}})$ be such that $\left(A: R_{E}\right) \neq 0,(A$ : $\left.R_{E^{\prime}}\right) \neq 0$. Then $\mathbf{c}_{E}=\mathbf{c}_{E^{\prime}}$.

By the proof of (ii) we see that there exists $x \in \mathbf{c}_{E}$ such that $\left(A: r_{x}\right) \neq 0$; similarly, there exists $x^{\prime} \in \mathbf{c}_{E^{\prime}}$ such that $\left(A: r_{x^{\prime}}\right) \neq 0$. Using this and (d) we deduce $\left(A: r_{x}\right)>0,\left(A: r_{x^{\prime}}\right)>0$. It follows that $\left(r_{x}: r_{x^{\prime}}\right)>0$. (By (d), $\left(r_{x}: r_{x^{\prime}}\right)$ is a sum of terms in $\mathbf{N}$, at least one of which is $>0$.) Again by the proof of (ii) we have

$$
r_{x}=\sum_{E_{1} ; \mathbf{c}_{E_{1}}=\mathbf{c}_{E}} s_{E_{1}} R_{E_{1}}, r_{x^{\prime}}=\sum_{E_{2} ; \mathbf{c}_{E_{2}}=\mathbf{c}_{E^{\prime}}} s_{E_{2}}^{\prime} R_{E_{2}},
$$

where $s_{E_{1}} \in \mathbf{Q}, s_{E_{2}}^{\prime} \in \mathbf{Q}$. From $\left(r_{x}: r_{x^{\prime}}\right) \neq 0$ it follows that there exist $E_{1}, E_{2}$ such that $\mathbf{c}_{E_{1}}=\mathbf{c}_{E}, \mathbf{c}_{E_{2}}=\mathbf{c}_{E^{\prime}},\left(R_{E_{1}}: R_{E_{2}}\right) \neq 0$. From 44.7(l) we deduce that $E_{1}, E_{2}$ have isomorphic restrictions to $\mathbf{Q}[\mathbf{W}]$, hence $\mathbf{c}_{E_{1}}=\mathbf{c}_{E_{2}}$. It follows that $\mathbf{c}_{E}=\mathbf{c}_{E^{\prime}}$. This proves (e). 
Proposition 44.18. Recall that $D$ is assumed to have property $\mathfrak{A}$ and property $\tilde{\mathfrak{A}}$. Let $A \in \hat{D}^{u n}$.

(a) There exists a well-defined two-sided cell $\mathbf{c}_{A}^{\prime}$ in $\mathbf{W}$ such that whenever $E \in$ $\operatorname{Irr}(\tilde{\mathbf{W}})$ and $\left(A: R_{E}\right) \neq 0$, we have $\mathbf{c}_{E}=\mathbf{c}_{A}^{\prime}$. Moreover, we have $\epsilon\left(\mathbf{c}_{A}^{\prime}\right)=$ $\mathbf{c}_{A}^{\prime}$.

(b) We have $w_{0} \mathbf{c}_{A}^{\prime}=\mathbf{c}_{A}$ where $\mathbf{c}_{A}$ is as in 41.4.

(a) follows from 44.17(e) and 43.6(f). We prove (b). Recall (41.8) that

(c) $A \dashv \bar{K}_{D}^{x}$ for some $x \in \mathbf{c}_{A}$; if $x^{\prime} \in \mathbf{W}$ and $A \dashv \bar{K}_{D}^{x^{\prime}}$, then $x \preceq x^{\prime}$.

We show:

(d) if $E \in \operatorname{Irr}(\tilde{\mathbf{W}})$ is such that $\left(A: R_{E}\right) \neq 0$, then $\mathbf{c}_{A} \preceq w_{0} \mathbf{c}_{E}$.

Using 44.6(c) we see that

$$
\left.\left.|\mathbf{W}|^{-1} \sum_{i \in \mathbf{Z}} \sum_{x \in \mathbf{W}}(-1)^{i+\operatorname{dim} G_{\operatorname{tr}}} \operatorname{tr} \tilde{c}_{x \varpi}\right|_{v=1}, E\right)\left(A: H^{i}\left(\bar{K}_{D}^{x}\right)\right) \neq 0 .
$$

Hence there exist $x \in \mathbf{W}, i \in \mathbf{Z}$ such that $\operatorname{tr}\left(\left.\tilde{c}_{x \varpi}\right|_{v=1}, E\right) \neq 0$ and $\left(A: H^{i}\left(\bar{K}_{D}^{x}\right)\right) \neq 0$. Using (c) we deduce that $y \preceq x$ for some $y \in \mathbf{c}_{A}$. From the definitions we have

$$
\tilde{c}_{x \varpi}=(-1)^{l\left(w_{0} x\right)} \tilde{T}_{w_{0}} c_{w_{0} x \varpi}^{\dagger} .
$$

It follows that $\operatorname{tr}\left(\left.w_{0} c_{w_{0} x \varpi}^{\dagger}\right|_{v=1}, E\right) \neq 0$. Thus the action of $\left.c_{w_{0} x \varpi}^{\dagger}\right|_{v=1}$ on $E$ is $\neq 0$. Using 43.6(b) we see that $z \preceq w_{0} x$ for some $z \in \mathbf{c}_{E}$. Hence $x \preceq w_{0} z$. Since $y \preceq x$, we have $y \preceq w_{0} z$. Since $y \in \mathbf{c}_{A}$ we have $\mathbf{c}_{A} \preceq w_{0} \mathbf{c}_{E}$. This proves (d).

We show:

(e) There exists $E \in \operatorname{Irr}(\tilde{\mathbf{W}})$ such that $\left(A: R_{E}\right) \neq 0$ and $w_{0} \mathbf{c}_{E}=\mathbf{c}_{A}$.

Let $x$ be as in (c). We have $\sum_{j \in \mathbf{Z}}\left(A: H^{j}\left(\bar{K}_{D}^{x}\right)\right)(-v)^{j} \neq 0$. Using 6.7(c) we deduce that

$$
v^{\operatorname{dim} G+l(x)} \sum_{E \in \mathfrak{E}} b_{A, E} \operatorname{tr}\left(c_{x \varpi}, E^{v}\right) \neq 0 .
$$

Hence there exists $E \in \operatorname{Irr}(\tilde{\mathbf{W}})$ such that $\left(A: R_{E}\right) \neq 0$ and $\operatorname{tr}\left(c_{x \varpi}, E^{v}\right) \neq 0$ that is, $\operatorname{tr}\left(c_{x \varpi}^{\dagger},\left(E^{\dagger}\right)^{v}\right) \neq 0$. The last condition implies, in view of $43.6(\mathrm{~b})$ that $z \preceq x$ for some $z \in \mathbf{c}_{E^{\dagger}}=w_{0} \mathbf{c}_{E}$. Thus, $w_{0} \mathbf{c}_{E} \preceq \mathbf{c}_{A}$. Since $\mathbf{c}_{A} \preceq w_{0} \mathbf{c}_{E}$ by (d), it follows that $\mathbf{c}_{A}=w_{0} \mathbf{c}_{E}$. This proves (e).

From (e) we see that $w_{0} \mathbf{c}_{A}^{\prime}=\mathbf{c}_{A}$. The proposition is proved.

44.19. For any $\epsilon$-stable two-sided cell $\mathbf{c}$ of $\mathbf{W}$ let $\hat{D}_{\mathbf{c}}^{u n}$ be the category whose objects are those $A \in \hat{D}^{u n}$ such that $\mathbf{c}_{A}^{\prime}=\mathbf{c}$ (see 44.18 ) and let $\mathcal{K}^{\mathbf{c}}(D)$ be the subgroup of $\mathcal{K}^{u n}(D)$ generated by the various $A \in \hat{D}_{\mathbf{c}}^{u n}$ up to isomorphism. We have $\mathcal{K}^{\text {un }}(D)=\bigoplus_{\mathbf{c}} \mathcal{K}^{\mathbf{c}}(D)$ where $\mathbf{c}$ runs over the $\epsilon$-stable two-sided cells of $\mathbf{W}$. We show:

(a) $A \mapsto A^{\circ}$ (see 44.8(a)) induces a bijection between the set of isomorphism classes in $\hat{D}_{\mathbf{c}}^{u n}$ and the set of isomorphism classes in $\hat{D}_{w_{0} \mathbf{c}}^{u n}$; it also induces an isomorphism $\mathcal{K}^{\mathbf{c}}(D) \stackrel{\sim}{\longrightarrow} \mathcal{K}^{w_{0} \mathbf{c}}(D)$.

Let $A \in \hat{D}_{\mathbf{c}}^{u n}$. Then $\left(A: R_{E}\right) \neq 0$ for some $E \in \operatorname{Irr}(\tilde{\mathbf{W}})$ such that $\mathbf{c}_{E}=\mathbf{c}$. We have $\left(\mathbf{d}(A): \mathbf{d}\left(R_{E}\right)\right) \neq 0$ and $\left(A^{\circ}: R_{E \otimes \operatorname{sgn}}\right) \neq 0$ (see $\left.44.8(\mathrm{~d})\right)$. Thus $A^{\circ} \in \hat{D}_{\mathbf{c}_{E \otimes s g n}}^{u n}=$ $\hat{D}_{w_{0} \mathrm{c}}^{u n}$. The remaining statements of (a) are immediate. 
44.20. Let $I$ be a subset of $\mathbf{I}$ such that $\epsilon(I)=I$. We fix a two-sided cell $\mathbf{c}^{\prime}$ of $\mathbf{W}_{I}$ (see 26.1) such that $\epsilon\left(\mathbf{c}^{\prime}\right)=\mathbf{c}^{\prime}$. There is a unique two-sided cell $\mathbf{c}$ of $\mathbf{W}$ such that $\mathbf{c}^{\prime} \subset \mathbf{c}$; we must have $\epsilon(\mathbf{c})=\mathbf{c}$.

Let $\operatorname{Irr}_{\mathbf{c}}(\tilde{\mathbf{W}})=\left\{E \in \operatorname{Irr}(\tilde{\mathbf{W}}) ; \mathbf{c}_{E}=\mathbf{c}\right\}, \operatorname{Irr}_{\mathbf{c}^{\prime}}\left(\tilde{\mathbf{W}}_{I}\right)=\left\{E^{\prime} \in \operatorname{Irr}\left(\tilde{\mathbf{W}}_{I}\right) ; \mathbf{c}_{E^{\prime}}=\mathbf{c}^{\prime}\right\}$.

Let $\mathcal{R}_{\mathbf{c}}(\tilde{\mathbf{W}})$ be the subgroup of $\mathcal{R}(\tilde{\mathbf{W}})$ generated by the elements $\phi_{E}$ with $E \in$ $\operatorname{Irr}_{\mathbf{c}}(\tilde{\mathbf{W}})$. Let $\mathcal{R}_{\mathbf{c}^{\prime}}\left(\tilde{\mathbf{W}}_{I}\right)$ be the subgroup of $\mathcal{R}\left(\tilde{\mathbf{W}}_{I}\right)$ generated by the elements $\phi_{E^{\prime}}$ with $E^{\prime} \in \operatorname{Irr}_{\mathbf{c}^{\prime}}\left(\tilde{\mathbf{W}}_{I}\right)$. From $43.11(\mathrm{~b})$ we see that

$$
J_{\tilde{\mathbf{W}}_{I}}^{\tilde{\mathbf{W}}}: \mathcal{R}\left(\tilde{\mathbf{W}}_{I}\right) \rightarrow \mathcal{R}(\tilde{\mathbf{W}}) \text { satisfies } J_{\tilde{\mathbf{W}}_{I}}^{\tilde{\mathbf{W}}}\left(\mathcal{R}_{\mathbf{c}^{\prime}}\left(\tilde{\mathbf{W}}_{I}\right)\right) \subset \mathcal{R}_{\mathbf{c}}(\tilde{\mathbf{W}}) \text {. }
$$

Let $\mathcal{K}^{\mathbf{c}}(D)$ be as in 44.19. Similarly, we define $\mathcal{K}^{\mathbf{c}^{\prime}}\left(D^{\prime}\right)$. Define a $\mathbf{Q}$-linear map $p_{\mathbf{c}}: \mathbf{Q} \otimes \mathcal{K}^{u n}(D) \rightarrow \mathbf{Q} \otimes \mathcal{K}^{\mathbf{c}}(D)$ by $A \mapsto A$ if $A \in \hat{D}_{\mathbf{c}}^{u n}$ and $A \mapsto 0$ if $A \in \hat{D}^{u n}, \mathbf{c}_{A}^{\prime} \neq \mathbf{c}$; this restricts to a homomorphism $\mathcal{K}^{u n}(D) \rightarrow \mathcal{K}^{\mathbf{c}}(D)$. Note that for $E_{1} \in \operatorname{Irr}(\tilde{\mathbf{W}})$ we have $R_{E_{1}} \in \mathbf{Q} \otimes \mathcal{K}^{\mathbf{c}_{E_{1}}}(D)$, hence

(b) $p_{\mathbf{c}}\left(R_{E_{1}}\right)=R_{E_{1}}$ if $\mathbf{c}_{E_{1}}=\mathbf{c}$ and $p_{\mathbf{c}}\left(R_{E_{1}}\right)=0$ if $\mathbf{c}_{E_{1}} \neq \mathbf{c}$.

Let $E^{\prime} \in \operatorname{Irr}_{\mathbf{c}^{\prime}}\left(\tilde{\mathbf{W}}_{I}\right)$. We show:

$$
R_{J_{\mathbf{W}_{I}}^{\tilde{\mathbf{W}}}\left(\phi_{E^{\prime}}\right)}=p_{\mathbf{c}}\left(R_{{\text {ind } \tilde{\mathbf{W}}_{I}}^{\tilde{W}} E^{\prime}}\right) .
$$

By 44.7(o) and (b), both sides of (c) are integer combinations of elements of the form $R_{E_{1}}$ with $E_{1} \in \mathfrak{E}$. Hence (using $44.7(\mathrm{l})$ ) it is enough to show that for any $E_{1} \in \mathfrak{E}$, we have

$$
\left(R_{J_{\mathbf{W}_{I}}^{\tilde{\tilde{w}}}\left(\phi_{E^{\prime}}\right)}: R_{E_{1}}\right)=\left(p_{\mathbf{c}}\left(R_{\mathrm{ind}_{\mathbf{W}_{I}}^{\tilde{\tilde{W}}} E^{\prime}}\right): R_{E_{1}}\right) .
$$

If $\mathbf{c}_{E_{1}} \neq \mathbf{c}$, then from (b) we see that the right-hand side of (d) is zero; moreover, since $\phi_{E^{\prime}} \in \mathcal{R}_{\mathbf{c}^{\prime}}\left(\tilde{\mathbf{W}}_{I}\right)$ we have $J_{\tilde{\mathbf{W}}_{I}}^{\tilde{\mathbf{W}}}\left(\phi_{E^{\prime}}\right) \subset \mathcal{R}_{\mathbf{c}}(\tilde{\mathbf{W}})$ (see (a)), hence $R_{J_{\mathbf{W}_{I}}^{\tilde{\tilde{w}}}\left(\phi_{E^{\prime}}\right)} \in$ $\mathcal{K}^{\mathbf{c}}(D)$ so that the left-hand side of (d) is also zero. Thus, we may assume that $\mathbf{c}_{E_{1}}=\mathbf{c}$. In this case (d) can be rewritten in the form

$$
\left(R_{J_{\mathbf{W}_{I}}^{\tilde{\tilde{w}}}\left(\phi_{E^{\prime}}\right)}: R_{E_{1}}\right)=\left(R_{\mathrm{ind}_{\mathbf{w}_{I}}^{\tilde{\tilde{w}}} E^{\prime}}: R_{E_{1}}\right)
$$

or equivalently (using $44.7(1)$ ) in the form

$$
\begin{gathered}
\sum_{E \in \underline{\operatorname{Ir}}(\tilde{\mathbf{W}}) ; a_{E^{\prime}}=a_{E}}\left\langle E^{\prime}, E\right\rangle|\mathbf{W}|^{-1} \sum_{u \in \mathbf{W}} \operatorname{tr}(u \varpi, E) \operatorname{tr}\left(u \varpi, E_{1}\right) \\
=|\mathbf{W}|^{-1} \sum_{x \in \mathbf{W}} \operatorname{tr}\left(x \varpi, \operatorname{ind}_{\tilde{\mathbf{W}}_{I}}^{\tilde{\mathbf{W}}} E^{\prime}\right) \operatorname{tr}\left(x \varpi, E_{1}\right) .
\end{gathered}
$$

The right-hand side of (e) can be rewritten as $\left|\mathbf{W}_{I}\right|^{-1} \sum_{z \in \mathbf{W}_{I}} \operatorname{tr}\left(z \varpi, E^{\prime}\right) \operatorname{tr}\left(z \varpi, E_{1}\right)$; substituting $\operatorname{tr}\left(z \varpi, E_{1}\right)=\sum_{E_{1}^{\prime} \in \underline{\operatorname{Irr}}\left(\tilde{\mathbf{W}}_{I}\right)}\left\langle E_{1}^{\prime}, E_{1}\right\rangle \operatorname{tr}\left(z \varpi, E^{\prime}\right)$ (see $\left.43.9(\mathrm{a})\right)$ this becomes

$$
\begin{aligned}
& \left|\mathbf{W}_{I}\right|^{-1} \sum_{z \in \mathbf{W}_{I}} \operatorname{tr}\left(z \varpi, E^{\prime}\right) \sum_{E_{1}^{\prime} \in \underline{\operatorname{Irr}}\left(\tilde{\mathbf{W}}_{I}\right)}\left\langle E_{1}^{\prime}, E_{1}\right\rangle \operatorname{tr}\left(z \varpi, E_{1}^{\prime}\right) \\
& =\sum_{E_{1}^{\prime} \in \underline{\operatorname{Irr}}\left(\tilde{\mathbf{W}}_{I}\right)}\left\langle E_{1}^{\prime}, E_{1}\right\rangle \alpha\left(E^{\prime}, E_{1}^{\prime}\right)=\left\langle E^{\prime}, E_{1}\right\rangle-\left\langle E^{\prime} \otimes \iota, E_{1}\right\rangle
\end{aligned}
$$

where $\alpha\left(E^{\prime}, E_{1}^{\prime}\right)$ is 1 if $E^{\prime} \cong E_{1}^{\prime}$, is -1 if $E^{\prime} \cong E_{1}^{\prime} \otimes \iota$ and is 0 otherwise. Now in the left-hand side of (e) the second sum is zero unless $E$ is isomorphic to $E_{1}$ or to 
$E_{1} \otimes \iota$ in which case we have automatically $a_{E^{\prime}}=a_{E}$ (since $a_{E}=a_{E_{1}}$ ). Thus the left-hand side of (e) is equal to

$$
\begin{aligned}
& \sum_{E \in \underline{\operatorname{Ir}}(\tilde{\mathbf{W}})}\left\langle E^{\prime}, E\right\rangle|\mathbf{W}|^{-1} \sum_{u \in \mathbf{W}} \operatorname{tr}(u \varpi, E) \operatorname{tr}\left(u \varpi, E_{1}\right) \\
= & \sum_{E \in \underline{\operatorname{Ir}}(\tilde{\mathbf{W}})}\left\langle E^{\prime}, E\right\rangle \alpha\left(E, E_{1}\right)=\left\langle E^{\prime}, E_{1}\right\rangle-\left\langle E^{\prime}, E_{1} \otimes E\right\rangle .
\end{aligned}
$$

This proves (e) and hence (c).

For any $A^{\prime} \in \hat{D}_{\mathbf{c}^{\prime}}^{\prime \text { un }}$ we set $\operatorname{tind}_{D^{\prime}}^{D}\left(A^{\prime}\right)=p_{\mathbf{c}}\left(\operatorname{ind}_{D^{\prime}}^{D}(A)\right)$, (see 44.13). Now $A^{\prime} \mapsto$ $\operatorname{tind}_{D^{\prime}}^{D}\left(A^{\prime}\right)$ defines a group homomorphism $\mathcal{K}^{\mathbf{c}^{\prime}}\left(D^{\prime}\right) \rightarrow \mathcal{K}^{\mathbf{c}}(D)$ and a Q-linear map $\mathbf{Q} \otimes \mathcal{K}^{\mathbf{c}^{\prime}}\left(D^{\prime}\right) \rightarrow \mathbf{Q} \otimes \mathcal{K}_{\mathbf{c}}(D)$; these are denoted again by $\operatorname{tind}_{D^{\prime}}^{D}$.

Let $\phi^{\prime} \in \mathcal{R}_{\mathbf{c}^{\prime}}\left(\tilde{\mathbf{W}}_{I}\right)$. We show:

$$
\operatorname{tind}_{D^{\prime}}^{D}\left(R_{\phi^{\prime}}\right)=R_{J_{\mathbf{W}_{I}}^{\tilde{\mathbf{w}}}\left(\phi^{\prime}\right)} .
$$

We may assume that $\phi^{\prime}=\phi_{E^{\prime}}$ where $E^{\prime} \in \operatorname{Irr}_{\mathbf{c}^{\prime}}\left(\tilde{\mathbf{W}}_{I}\right)$. From the definitions we have $R_{\phi_{E^{\prime}}} \in \mathbf{Q} \otimes \mathcal{K}^{\mathbf{c}^{\prime}}\left(D^{\prime}\right)$ and $\operatorname{tind}_{D^{\prime}}^{D}\left(R_{\phi_{E^{\prime}}}\right) \in \mathbf{Q} \otimes \mathcal{K}^{\mathbf{c}}(D)$. Applying $p_{\mathbf{c}}$ to the identity

$$
\operatorname{ind}_{D^{\prime}}^{D}\left(R_{\phi_{E^{\prime}}}\right)=R_{\text {ind }_{\tilde{\mathbf{w}}_{I}}^{\tilde{\tilde{w}}} E^{\prime}} \in \mathcal{K}_{\mathbf{Q}}^{u n}(D)
$$

(see 44.14(a)) we obtain

$$
\operatorname{tind}_{D^{\prime}}^{D}\left(R_{\phi_{E^{\prime}}}\right)=p_{\mathbf{c}}\left(R_{\operatorname{ind}_{\tilde{\mathbf{w}}_{I}}^{\tilde{\mathbf{W}}} E^{\prime}}\right)
$$

Now (f) follows from (c).

For any $x \in \mathbf{c}$ we have $\aleph_{x \varpi} \in \mathcal{R}_{\mathbf{c}}(\tilde{\mathbf{W}})$. Similarly, for any $x \in \mathbf{c}^{\prime}$ we have $\aleph_{x \varpi}^{I I} \in \mathcal{R}_{\mathbf{c}^{\prime}}\left(\tilde{\mathbf{W}}_{I}\right)$. Combining (f) (with $\phi^{\prime}=\aleph_{x \varpi}^{I}, x \in \mathbf{c}^{\prime}$ ) with $43.10(\mathrm{c})$ we see that

$$
\operatorname{tind}_{D^{\prime}}^{D}\left(R_{\aleph_{x \varpi}^{I}}\right)=R_{\aleph_{x \varpi}^{I}} .
$$

We define a homomorphism ' $J_{\tilde{\mathbf{W}}}^{\tilde{\mathbf{W}}_{I}}: \mathcal{R}(\tilde{\mathbf{W}}) \rightarrow \mathcal{R}\left(\tilde{\mathbf{W}}_{I}\right)$ by

$$
{ }^{\prime} J_{\tilde{\mathbf{W}}}^{\tilde{\mathbf{W}}_{I}}\left(\phi_{E}\right)=\sum_{E^{\prime} \in \underline{\operatorname{Irr}}\left(\tilde{\mathbf{W}}_{I}\right) ; a_{E^{\prime}}=a_{E}}\left\langle E^{\prime}, E\right\rangle \phi_{E^{\prime}}
$$

for any $E \in \operatorname{Irr}(\tilde{\mathbf{W}})$.

Let $\phi \in \mathcal{R}_{\mathbf{c}}(\tilde{\mathbf{W}})$ and let $A^{\prime} \in \hat{D}_{\mathbf{c}^{\prime}}^{\prime \text { un }}$. We show:

$$
\left(\operatorname{tind}_{D^{\prime}}^{D}\left(A^{\prime}\right): R_{\phi}\right)=\left(A^{\prime}: R_{J_{\tilde{\mathbf{W}}}^{\tilde{\mathbf{w}}_{I}(\phi)}}^{\tilde{\tilde{N}}_{I}}\right) .
$$

We may assume that $\phi=\phi_{E}$ where $E \in \operatorname{Irr}_{\mathbf{c}}(\tilde{\mathbf{W}})$. By the definition of $\operatorname{tind}_{D^{\prime}}^{D}\left(A^{\prime}\right)$, the left-hand side of $(\mathrm{h})$ is equal to $\left.\operatorname{(ind}_{D^{\prime}}^{D}\left(A^{\prime}\right): R_{E}\right)$. From the second equality in 43.9 (a) we see that

$$
R_{\left.E\right|_{\tilde{\mathbf{W}}_{I}}}=\sum_{E^{\prime} \in \underline{\operatorname{Irr}}\left(\tilde{\mathbf{W}}_{I}\right)}\left\langle E^{\prime}, E\right\rangle R_{E^{\prime}}
$$

By 43.9(b) we may restrict the previous sum to those $E^{\prime}$ such that $a_{E^{\prime}} \leq a_{E}$; moreover, for $E^{\prime}$ such that $a_{E^{\prime}}<a_{E}$ we have $\mathbf{c}_{E^{\prime}} \neq \mathbf{c}^{\prime}$. Thus we have $R_{\left.E\right|_{\tilde{\mathbf{w}}_{I}}}=$ $R_{\left.J_{J_{\tilde{\mathbf{w}}}(\phi)}^{\tilde{\tilde{w}}_{I}(}\right)}$ plus a linear combination of $A^{\prime \prime} \in \hat{D}^{\prime \text { un }}$ with $\mathbf{c}_{A^{\prime \prime}}^{\prime} \neq \mathbf{c}^{\prime}$. We see that

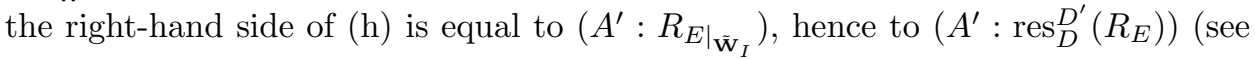


44.12(e)) and (h) is equivalent to $\left(\operatorname{ind}_{D^{\prime}}^{D}\left(A^{\prime}\right): R_{E}\right)=\left(A^{\prime}: \operatorname{res}_{D}^{D^{\prime}}\left(R_{E}\right)\right)$; but this follows from $44.12(\mathrm{~d})$. This proves $(\mathrm{h})$.

44.21. We preserve the setup of 44.20 . We assume that

(i) for any $E^{\prime} \in \operatorname{Irr}_{\mathbf{c}^{\prime}}\left(\tilde{\mathbf{W}}_{I}\right)$ there exists a unique $E \in \operatorname{Irr}_{\mathbf{c}}(\tilde{\mathbf{W}})$ (up to isomorphism) such that $\left\langle E^{\prime}, E\right\rangle \neq 0$; moreover, we then have $\left\langle E^{\prime}, E\right\rangle=1$;

(ii) for any $E \in \operatorname{Irr}_{\mathbf{c}}(\tilde{\mathbf{W}})$ there exists a unique $E^{\prime} \in \operatorname{Irr}_{\mathbf{c}^{\prime}}\left(\tilde{\mathbf{W}}_{I}\right)$ (up to isomorphism) such that $\left\langle E^{\prime}, E\right\rangle \neq 0$; moreover, we then have $\left\langle E^{\prime}, E\right\rangle=1$.

Note that the $E^{\prime} \mapsto E$ in (i) and $E \mapsto E^{\prime}$ in (ii) defined inverse bijections $E^{\prime} \leftrightarrow E$ between the sets of isomorphism classes of objects in $\operatorname{Irr}_{\mathbf{c}^{\prime}}\left(\tilde{\mathbf{W}}_{I}\right)$ and $\operatorname{Irr}_{\mathbf{c}}(\tilde{\mathbf{W}})$. If $E^{\prime} \leftrightarrow E$, then

$$
\begin{aligned}
& J_{\tilde{\mathbf{W}}_{I}}^{\tilde{\mathbf{W}}}\left(\phi_{E^{\prime}}\right)=\phi_{E}, \\
& { }^{\prime} J_{\tilde{\mathbf{W}}}^{\tilde{\mathbf{W}}_{I}}\left(\phi_{E}\right)=\phi_{E^{\prime}}+\text { linear combination of elements } \phi_{E^{\prime \prime}} \text { with } \\
& E^{\prime \prime} \in \operatorname{Irr}\left(\tilde{\mathbf{W}}_{I}\right)-\operatorname{Irr}_{\mathbf{c}^{\prime}}\left(\tilde{\mathbf{W}}_{I}\right) .
\end{aligned}
$$

The second equality in (a) is obvious. To prove the first equality in (a) we consider $\tilde{E} \in \operatorname{Irr}(\mathbf{W})$ such that $a_{E^{\prime}}=a_{\tilde{E}}$ and $\left\langle E^{\prime}, \tilde{E}\right\rangle \neq 0$. It is enough to show that $\tilde{E}=E$. By 43.11 (b) we have $\mathbf{c}_{\tilde{E}}=\mathbf{c}$. Using (i) we see that $\tilde{E}=E$, as required.

We show:

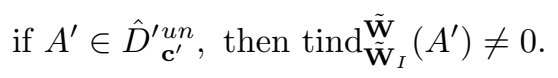

Assume that $\operatorname{tind}_{\tilde{\mathbf{W}}_{I}}^{\tilde{\tilde{W}}}\left(A^{\prime}\right)=0$. From $44.20(\mathrm{~h})$ we deduce $\left(A^{\prime}: R_{J_{J_{\tilde{\mathbf{W}}}}^{\tilde{\mathbf{W}}_{I}}}\right)=0$ for any $E \in \operatorname{Irr}_{\mathbf{c}}(\tilde{\mathbf{W}})$. Thus, for any $E^{\prime} \in \operatorname{Irr}_{\mathbf{c}^{\prime}}\left(\tilde{\mathbf{W}}_{I}\right)$ we have $\left(A^{\prime}: R_{\phi_{E^{\prime}}}\right)=0$ (see (a)). This contradicts $44.7(\mathrm{k})$ for $D^{\prime}$. This proves (b).

We show:

$$
\text { if } A^{\prime} \in \hat{D}_{\mathbf{c}^{\prime}}^{\prime \text { un }} \text {, then } A:=\operatorname{tind}_{\tilde{\mathbf{W}}_{I}}^{\tilde{\mathbb{W}}}\left(A^{\prime}\right) \text { is a single object of } \hat{D}_{\mathbf{c}}^{u n} .
$$

By $44.7(\mathrm{k})$ we can find $E^{\prime} \in \operatorname{Irr}\left(\tilde{\mathbf{W}}_{I}\right)$ such that $\left(A^{\prime}: R_{E^{\prime}}\right) \neq 0$. We have necessarily $E^{\prime} \in \operatorname{Irr}_{\mathbf{c}^{\prime}}\left(\tilde{\mathbf{W}}_{I}\right)$. By 43.10(b), $R_{E^{\prime}}$ is a Q-linear combination of elements $R_{\aleph_{x_{m}}^{I}}$ such that $\operatorname{tr}\left(t_{x} \varpi, E^{\infty}\right) \neq 0$ (and in particular $x \in \mathbf{c}^{\prime}$ ). Hence there exists $x \in \mathbf{c}^{\prime}$ such that $\left(A^{\prime}: R_{\aleph_{x \varpi}^{I}}\right) \neq 0$. By $44.20(\mathrm{~d})$ we have

$$
R_{\aleph_{x \varpi}^{I}}=n_{1} A_{1}+n_{2} A_{2}+\cdots+n_{r} A_{r}
$$

where $A_{i} \in \hat{D}^{\prime \text { un }}$ are nonisomorphic to each other and $n_{i} \in \mathbf{Z}_{>0}$; we can assume that $A_{1}=A^{\prime}$. We have:

$$
\begin{aligned}
& { }^{\prime} J_{\tilde{\mathbf{W}}}^{\tilde{\mathbf{W}}_{I}}\left(\aleph_{x \varpi}\right)=\aleph_{x \varpi}^{I}+\text { linear combination of elements } \phi_{E^{\prime \prime}} \text { with } \\
& E^{\prime \prime} \in \operatorname{Irr}\left(\tilde{\mathbf{W}}_{I}\right)-\operatorname{Irr}_{\mathbf{c}^{\prime}}\left(\tilde{\mathbf{W}}_{I}\right) .
\end{aligned}
$$

Using (a) we see that this is equivalent to the identity $\operatorname{tr}\left(t_{x} \varpi, E^{\infty}\right)=\operatorname{tr}\left(t_{x} \varpi, E^{\prime \infty}\right)$ (for any $E^{\prime} \leftrightarrow E$ as above) which follows from $43.10(\mathrm{c})$. For $i, j$ in $[1, r]$ we set 


$$
\begin{aligned}
& x_{i, j}=\left(\operatorname{tind}_{\tilde{\mathbf{W}}_{I}}^{\tilde{\mathbf{W}}}\left(A_{i}\right): \operatorname{tind}_{\tilde{\mathbf{W}}_{I}}^{\tilde{\mathbf{W}}}\left(A_{j}\right)\right) . \text { We have: } \\
& \left.\sum_{i, j \in[1, r]} n_{i} n_{j} x_{i, j}=\left(\operatorname{tind}{\tilde{\tilde{\mathbf{W}}_{I}}}_{\tilde{\mathbf{W}}}^{\tilde{\mathbf{W}}}\left(R_{\aleph_{x \varpi}^{I}}\right): \operatorname{tind}_{\tilde{\mathbf{W}}_{I}}^{\tilde{\mathbf{W}}}\left(R_{\aleph_{x \varpi}^{I}}\right)\right)=\left(\operatorname{tind} \tilde{\mathbf{W}}_{\tilde{\mathbf{W}}_{I}} \tilde{\mathbf{W}}_{\aleph_{x \varpi}^{I}}\right): R_{\aleph_{x \varpi}}\right)
\end{aligned}
$$

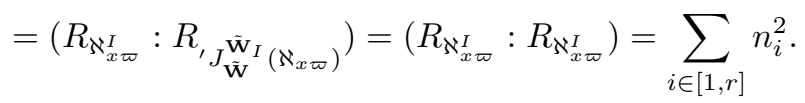

(The first equality comes from (d); the second equality comes from $44.20(\mathrm{~g})$; the third equality comes from $44.20(\mathrm{~h})$; the fourth equality comes from (e); the fifth equality comes from (d).) Since $\operatorname{tind}_{\tilde{\mathbf{W}}_{I}}^{\tilde{\mathbf{W}}}\left(A_{i}\right)$ is an $\mathbf{N}$-linear combination of objects in $\hat{D}^{u n}$ and is $\neq 0$ by (b), we see that $\left(\operatorname{tind}_{\tilde{\mathbf{W}}_{I}}^{\tilde{\mathbf{W}}}\left(A_{i}\right): \operatorname{tind}_{\tilde{\mathbf{W}}_{I}}^{\tilde{\mathbf{W}}}\left(A_{j}\right)\right)$ is $\geq 1$ if $i=j$ and is $\geq 0$ if $i \neq j$. Hence from the equality $\sum_{i, j \in[1, r]} n_{i} n_{j} x_{i, j}=\sum_{i \in[1, r]} n_{i}^{2}$ it follows that $x_{i, j}=1$ if $i=j$ and $x_{i, j}=0$ if $i \neq j$. Since $A^{\prime}=A_{1}$ we see that (c) holds.

We show:

(f) If $A_{1}, A_{2}$ are objects of $\hat{D}_{\mathbf{c}^{\prime}}^{\prime \text { un }}$ and $A:=\operatorname{tind}_{\tilde{\mathbf{W}}_{I}}^{\tilde{\mathbf{W}}}\left(A_{1}\right)=\operatorname{tind}_{\tilde{\mathbf{W}}_{I}}^{\tilde{\mathbf{W}}}\left(A_{2}\right)$, then $A_{1} \cong A_{2}$.

Assume that $A_{1} \not \approx A_{2}$. Let $E^{\prime} \in \operatorname{Irr}_{\mathbf{c}^{\prime}}\left(\tilde{\mathbf{W}}_{I}\right)$. We can find $E \in \operatorname{Irr}_{\mathbf{c}}(\tilde{\mathbf{W}})$ such that $\left\langle E^{\prime}, E\right\rangle=1$. For $i=1,2$ we have:

$$
\left(A: R_{E}\right)=\left(\operatorname{tind}_{\tilde{\mathbf{W}}_{I}}^{\tilde{\tilde{W}}}\left(A_{i}\right): R_{E}\right)=\left(A_{i}: R_{J_{\tilde{\mathbf{W}}}}^{\tilde{\mathbf{W}}_{I}\left(\phi_{E}\right)}\right)=\left(A_{i}: R_{E^{\prime}}\right) .
$$

(The second equality holds by $44.20(\mathrm{~h})$; the third equality holds by (a).) Thus we have $\left(A_{1}: R_{E^{\prime}}\right)=\left(A_{2}: R_{E^{\prime}}\right)$ for any $E^{\prime} \in \operatorname{Irr}_{\mathbf{c}^{\prime}}\left(\tilde{\mathbf{W}}_{I}\right)$. This implies that $\left(A_{1}: R_{\aleph_{x \varpi}^{I}}\right)=\left(A_{2}: R_{\aleph_{x \varpi \varpi}^{I}}\right)$ for any $x \in \mathbf{W}_{I}$. We can choose $x \in \mathbf{c}^{\prime}$ such that $\left(A_{1}: R_{\aleph_{x \varpi}^{I}}^{I}\right) \neq 0$. Then we have also $\left(A_{2}: R_{\aleph_{x \varpi}^{I}}\right) \neq 0$. We can assume that $A_{1}, A_{2}$ are the first two terms in the right-hand side of $(\mathrm{d})$. But in the proof of (c) we have seen that $\left(\operatorname{tind}_{\tilde{\mathbf{W}}_{I}}^{\tilde{\mathbf{W}}}\left(A_{1}\right): \operatorname{tind}_{\tilde{\mathbf{W}}_{I}}^{\tilde{\mathbf{W}}}\left(A_{2}\right)\right)=0$. This contradicts the assumption that $\operatorname{tind}_{\tilde{\mathbf{W}}_{I}}^{\tilde{\mathbf{W}}}\left(A_{1}\right)=\operatorname{tind}_{\tilde{\mathbf{W}}_{I}}^{\tilde{\mathbf{W}}}\left(A_{2}\right)$ which is $\neq 0$ by (b). This proves (f).

We show:

(g) If $A \in \hat{D}_{\mathbf{c}}^{u n}$, then there exists $A^{\prime} \in \hat{D}_{\mathbf{c}^{\prime}}^{\prime u n}$ such that $A=\operatorname{tind}_{\tilde{\mathbf{W}}_{I}}^{\tilde{\mathbb{W}}}\left(A^{\prime}\right)$.

By $44.7(\mathrm{k})$ we can find $E \in \operatorname{Irr}(\tilde{\mathbf{W}})$ such that $\left(A: R_{E}\right) \neq 0$. We have necessarily $E \in \operatorname{Irr}_{\mathbf{c}}(\tilde{\mathbf{W}})$. Let $E^{\prime} \in \operatorname{Irr}_{\mathbf{c}^{\prime}}\left(\tilde{\mathbf{W}}_{I}\right)$ be such that $E^{\prime} \leftrightarrow E$. By 44.20(f) we have $0 \neq\left(A: R_{E}\right)=\left(A: R_{J_{\mathbf{W}_{I}}^{\tilde{\tilde{W}}}\left(E^{\prime}\right)}\right)=\left(A: \operatorname{tind}_{\tilde{\mathbf{W}}_{I}} \tilde{\tilde{W}}_{E^{\prime}}\left(R_{E^{\prime}}\right)\right)$. Hence there exists $A^{\prime} \in$ $\hat{D}_{\mathbf{c}^{\prime}}^{\prime u n}$ such that $\left(A^{\prime}: R_{E^{\prime}}\right) \neq 0$ and $\left(A: \operatorname{tind}_{\tilde{\mathbf{W}}_{I}}^{\tilde{\mathbf{W}}}\left(A^{\prime}\right)\right) \neq 0$. This implies that $A=\operatorname{tind}_{\tilde{\mathbf{W}}_{I}}^{\tilde{\tilde{W}}}\left(A^{\prime}\right)$. This proves $(\mathrm{g})$.

Combining (c), (f), (g), and using 44.20(h) and (a), we obtain the following result:

(h) $A^{\prime} \mapsto \operatorname{tind}_{\tilde{\mathbf{W}}_{I}}^{\tilde{\tilde{\mathbf{W}}}}\left(A^{\prime}\right)$ defines a bijection between the set of isomorphism classes in $\hat{D}_{\mathbf{c}^{\prime}}^{\prime}$ un and the set of isomorphism classes in $\hat{D}_{\mathbf{c}}^{u n}$; this bijection has the following property: for any $E \in \operatorname{Irr}(\tilde{\mathbf{W}})$ and any $A^{\prime} \in \hat{D}_{\mathbf{c}^{\prime}}^{\prime}$ un we have $\left(\operatorname{tind}_{\tilde{\mathbf{W}}_{I}}^{\tilde{\mathbf{W}}}\left(A^{\prime}\right): R_{E}\right)=0$ if $E \notin \operatorname{Irr}_{\mathbf{c}}(\tilde{\mathbf{W}})$ and $\left(\operatorname{tind}_{\tilde{\mathbf{W}}_{I}}^{\tilde{\mathbf{W}}}\left(A^{\prime}\right): R_{E}\right)=\left(A^{\prime}: R_{E^{\prime}}\right)$ where $E^{\prime} \in \operatorname{Irr}_{\mathbf{c}^{\prime}}\left(\tilde{\mathbf{W}}_{I}\right)$ is defined uniquely by $\left\langle E^{\prime}, E\right\rangle=1$. 


\section{Reductions}

45.1. In this section we show that the problem of classifying the unipotent character sheaves on $D$ can be reduced to the analogous problem in the case where $G^{0}$ is simple and $\mathcal{Z}_{G}=\{1\}$.

Let $\tau: G_{s c}^{0} \rightarrow G^{0}$ be a simply connected covering of the derived group of $G^{0}$. Let $\widetilde{G^{0}}=\mathcal{Z}_{G^{0}}^{0} \times G_{s c}^{0}$. The homomorphism $\psi: \widetilde{G^{0}} \rightarrow G^{0},(z, g) \mapsto z \tau(g)$ is surjective with finite kernel which may be identified with $\left\{z \in \mathcal{Z}_{G_{s c}^{0}} ; \tau(z) \in \mathcal{Z}_{G^{0}}^{0}\right\}$. Let $\mathfrak{s}\left(G^{0}\right)$ be the category whose objects are the local systems $\mathcal{E}$ of rank 1 on $G^{0}$ such that for some $\mathcal{E}_{0} \in \mathfrak{s}\left(\mathcal{Z}_{G^{0}}^{0}\right)$ we have $\psi^{*} \mathcal{E} \cong \mathcal{E}_{0} \otimes \overline{\mathbf{Q}}_{l}$ or equivalently $\mathcal{E}$ is a direct summand of the local system $\psi_{!}\left(\mathcal{E}_{0} \otimes \overline{\mathbf{Q}}_{l}\right)$. (When $G^{0}$ is a torus this definition of $\mathfrak{s}\left(G^{0}\right)$ agrees with that in 28.1.) Let $\mathcal{E} \in \mathfrak{s}\left(G^{0}\right)$. We show:

(a) $\mathcal{E}$ is $G^{0}$-equivariant for the conjugation action of $G^{0}$ on $G^{0}$.

(b) $\mathcal{E}$ is $G_{s c}^{0} \times G_{s c}^{0}$-equivariant for the $G_{s c}^{0} \times G_{s c}^{0}$-action on $G^{0}$ given by $\left(x_{1}, x_{2}\right)$ : $g \mapsto \tau\left(x_{1}\right) g \tau\left(x_{2}^{-1}\right)$.

(c) For any $x \in G^{0}$ we have $L_{x}^{*} \mathcal{E} \cong \mathcal{E}$ where $L_{x}: G^{0} \rightarrow G^{0}$ is given by $g \mapsto x g$. Let $\mathcal{E}_{0} \in \mathfrak{s}_{n}\left(\mathcal{Z}_{G^{0}}^{0}\right)$ be such that $\mathcal{E}$ is a direct summand of $\psi_{!}\left(\mathcal{E}_{0} \otimes \overline{\mathbf{Q}}_{l}\right)$. The $G^{0}$ action on $\widetilde{G^{0}}$ given by $y:(z, x) \mapsto \tilde{y}(z, x) \tilde{y}^{-1}$ (where $\left.\tilde{y} \in \psi^{-1}(y)\right)$ is well defined and is compatible under $\psi$ with the conjugation action of $G^{0}$ on $G^{0}$; moreover, $\mathcal{E}_{0} \otimes \overline{\mathbf{Q}}_{l}$ is $G^{0}$-equivariant. Hence $\psi_{!}\left(\mathcal{E}_{0} \otimes \overline{\mathbf{Q}}_{l}\right)$ is $G^{0}$-equivariant and (a) holds. The $G_{s c}^{0} \times G_{s c}^{0}$-action on $\widetilde{G^{0}}$ given by $\left(x_{1}, x_{2}\right):(z, x) \mapsto\left(z, x_{1} x x_{2}^{-1}\right)$ is compatible under $\psi$ with the $G_{s c}^{0} \times G_{s c}^{0}$-action on $G^{0}$ (as in (b)) and $\mathcal{E}_{0} \otimes \overline{\mathbf{Q}}_{l}$ is $G_{s c}^{0} \times G_{s c}^{0}$-equivariant. Hence $\psi_{!}\left(\mathcal{E}_{0} \otimes \overline{\mathbf{Q}}_{l}\right)$ is $G_{s c}^{0} \times G_{s c}^{0}$-equivariant and (b) holds. We prove (c). The $\widetilde{G^{0}}$-action on $\widetilde{G^{0}}$ given by $(z, x):\left(z^{\prime}, x^{\prime}\right) \mapsto\left(z^{n} z^{\prime}, x x^{\prime}\right)$ is compatible under $\psi$ with the $\widetilde{G^{0}}$-action on $G^{0}$ given by $(z, x): g \mapsto z^{n} \tau(x) g$ and $\mathcal{E}_{0} \otimes \overline{\mathbf{Q}}_{l}$ is $\widetilde{G^{0}}$-equivariant. Hence $\psi_{!}\left(\mathcal{E}_{0} \otimes \overline{\mathbf{Q}}_{l}\right)$ is $\widetilde{G^{0}}$-equivariant. Since the map $\widetilde{G^{0}} \rightarrow G^{0},(z, x) \mapsto z^{n} \tau(x)$ is surjective, we see that (c) holds.

Let $B^{*}, T$ be as in 28.5. Let $h: T \rightarrow G^{0}$ be the inclusion; let $\tilde{T}=\tau^{-1}(T)$ (a maximal torus of $G_{s c}^{0}$ ). Let $\tau_{T}: \tilde{T} \rightarrow T, \psi_{T}: \mathcal{Z}_{G^{0}}^{0} \times \tilde{T} \rightarrow T$ be the restrictions of $\tau, \psi$. Let $\mathfrak{s}(T)^{1}$ be the category whose objects are the local systems $\mathcal{E}^{\prime}$ in $\mathfrak{s}(T)$ which satisfy one of the following four equivalent conditions:

(i) for some $\mathcal{E}_{0} \in \mathfrak{s}\left(\mathcal{Z}_{G^{0}}^{0}\right)$ we have $\psi_{T}^{*} \mathcal{E}^{\prime} \cong \mathcal{E}_{0} \otimes \overline{\mathbf{Q}}_{l}$;

(ii) $\mathcal{E}^{\prime}$ is a direct summand of the local system $\psi_{T !}\left(\mathcal{E}_{0} \otimes \overline{\mathbf{Q}}_{l}\right)$;

(iii) $\tau_{T}^{*} \mathcal{E}^{\prime} \cong \overline{\mathbf{Q}}_{l}$;

(iv) for any coroot $f: \mathbf{k}^{*} \rightarrow T$ we have $f^{*} \mathcal{E}^{\prime} \cong \overline{\mathbf{Q}}_{l}$.

From the definitions we see that:

(d) $\mathcal{E} \mapsto \mathcal{E}_{T}:=h^{*} \mathcal{E}$ is an equivalence of categories $\mathfrak{s}\left(G^{0}\right) \rightarrow \mathfrak{s}(T)^{1}$.

Let $\mathfrak{s}(\mathbf{T})^{1}$ be the category whose objects are the local systems $\mathcal{E}^{\prime}$ in $\mathfrak{s}(\mathbf{T})$ such that $\check{\alpha}^{*} \mathcal{E}^{\prime} \cong \overline{\mathbf{Q}}_{l}$ for any $\alpha \in R$ (see 28.3). We identify $T=\mathbf{T}$ as in 28.5. Then $\mathfrak{s}(T)^{1}$ becomes $\mathfrak{s}(\mathbf{T})^{1}$.

45.2. Let $d \in N_{D}\left(B^{*}\right) \cap N_{D}(T)$. There is a unique automorphism $\delta_{0}: G_{s c}^{0} \rightarrow G_{s c}^{0}$ such that $\tau\left(\delta_{0}(g)\right)=d^{-1} \tau(g) d$ for all $g \in G_{s c}^{0}$. Define an automorphism $\delta: \widetilde{G^{0}} \rightarrow$ $\widetilde{G^{0}}$ by $\delta(z, g)=\left(d^{-1} z d, \delta_{0}(g)\right)$. Then $\psi(\delta(y))=d^{-1} \psi(y) d$ for all $y \in \widetilde{G^{0}}$.

Let $\mathcal{E} \in \mathfrak{s}\left(G^{0}\right)$. Note that $\operatorname{Ad}\left(d^{-1}\right)^{*} \mathcal{E} \in \mathfrak{s}\left(G^{0}\right)$. Define $L_{d^{-1}}: D \rightarrow G^{0}$ by $g \mapsto d^{-1} g$. We set $\mathcal{E}_{D}=L_{d^{-1}}^{*} \mathcal{E}$, a local system of rank 1 on $D$. We show that the following three conditions are equivalent: 
(i) $\operatorname{Ad}\left(d^{-1}\right)^{*} \mathcal{E} \cong \mathcal{E}$

(ii) $\operatorname{Ad}\left(d^{-1}\right)^{*} \mathcal{E}_{T} \cong \mathcal{E}_{T}$;

(iii) the local system $\mathcal{E}_{D}$ on $D$ is $G^{0}$-equivariant for the conjugation action of $G^{0}$ on $D$.

Now (i), (ii) are equivalent by 45.1(d); moreover, if (i) or (iii) holds for some $d \in N_{D}\left(B^{*}\right) \cap N_{D}(T)$, then it holds for any $d \in N_{D}\left(B^{*}\right) \cap N_{D}(T)$ (by the $G^{0}$ equivariance of $\mathcal{E}$, see $45.1(\mathrm{a}))$.

Assume first that (i) holds. Let $\tilde{D}=\left\{\left(y, x^{\prime}\right) \in D \times \widetilde{G^{0}} ; d^{-1} y=\psi\left(x^{\prime}\right)\right\}$. Let $L^{\prime}: \tilde{D} \rightarrow \widetilde{G^{0}}, \psi^{\prime}: \tilde{D} \rightarrow D$ be the obvious projections. Let $\mathcal{E}_{0} \in \mathfrak{s}\left(\mathcal{Z}_{G^{0}}^{0}\right)$ be such that $\psi^{*} \mathcal{E} \cong \mathcal{E}_{0} \otimes \overline{\mathbf{Q}}_{l}$. Then

$$
\operatorname{Ad}\left(d^{-1}\right)^{*} \mathcal{E}_{0} \nabla \overline{\mathbf{Q}}_{l}=\delta^{*}\left(\mathcal{E}_{0} \nabla \overline{\mathbf{Q}}_{l}\right) \cong \delta^{*} \psi^{*} \mathcal{E} \cong \psi^{*} \operatorname{Ad}\left(d^{-1}\right)^{*} \mathcal{E} \cong \psi^{*} \mathcal{E} \cong \mathcal{E}_{0} \otimes \overline{\mathbf{Q}}_{l},
$$

hence $\operatorname{Ad}\left(d^{-1}\right)^{*} \mathcal{E}_{0} \cong \mathcal{E}_{0}$. By $28.2, \mathcal{E}_{0}$ is $\mathcal{Z}_{G^{0}}^{0}$-equivariant for the $\mathcal{Z}_{G^{0}}^{0}$-action on $\mathcal{Z}_{G^{0}}^{0}$ given by $z_{0}: z \mapsto d^{-1} z_{0} d z z_{0}^{-1}$. Hence $\mathcal{E}_{0} \otimes \overline{\mathbf{Q}}_{l}$ is $\widetilde{G^{0}}$-equivariant for the $\widetilde{G^{0}}$-action on $\widetilde{G^{0}}$ given by $x: x^{\prime} \mapsto \delta(x) x^{\prime} x^{-1}$. Define a $\widetilde{G^{0}}$-action on $\tilde{D}$ by $x:\left(y, x^{\prime}\right) \mapsto$ $\left(\psi(x) y \psi(x)^{-1}, \delta(x) x^{\prime} x^{-1}\right)$. This action is compatible under $\psi^{\prime}$ with the $\widetilde{G}^{0}$-action on $D$ given by $x: y \mapsto \psi(x) y \psi(x)^{-1}$ and is compatible under $L^{\prime}$ with the $\widetilde{G^{0}}$-action on $\widetilde{G^{0}}$ given by $x: x^{\prime} \mapsto \delta(x) x^{\prime} x^{-1}$. It follows that $L^{\prime *}\left(\mathcal{E}_{0} \otimes \overline{\mathbf{Q}}_{l}\right)$ is $\widetilde{G^{0}}$-equivariant and $\psi_{!}^{\prime} L^{\prime *}\left(\mathcal{E}_{0} \otimes \overline{\mathbf{Q}}_{l}\right)=L_{d^{-1}}^{*} \psi_{!}\left(\mathcal{E}_{0} \otimes \overline{\mathbf{Q}}_{l}\right)$ is $\widetilde{G^{0}}$-equivariant. Since $L_{d^{-1}}^{*} \mathcal{E}$ is a direct summand of $L_{d^{-1}}^{*} \psi_{!}\left(\mathcal{E}_{0} \otimes \overline{\mathbf{Q}}_{l}\right)$, we see that $L_{d^{-1}}^{*} \mathcal{E}$ is $\widetilde{G^{0}}$-equivariant. Since $\widetilde{G^{0}}$ acts on $D$ through its quotient $G^{0}$, we see that ker $\psi$ acts naturally on the stalk of $L_{d^{-1}}^{*} \mathcal{E}$ at $y \in D$ through a character $\chi$ which is independent of $y$. To show that $L_{d^{-1}}^{*} \mathcal{E}$ is $G^{0}$-equivariant it is enough to show that $\chi=1$. Let $\tilde{T}=\psi^{-1}(T)$, a maximal torus of $\widetilde{G^{0}}$. Then $\left.L_{\delta^{-1}}^{*} \mathcal{E}\right|_{d T}$ is $\tilde{T}$-equivariant (for the restriction of the $\widetilde{G^{0}}$-action to $\tilde{T})$. Since ker $\psi \subset \tilde{T}, \chi$ is determined by the $\tilde{T}$-equivariant structure of $\left.L_{\delta^{-1}}^{*} \mathcal{E}\right|_{d T}$. To show that $\chi=1$ it is then enough to show that $\left.L_{\delta^{-1}}^{*} \mathcal{E}\right|_{d T}$ is $T$-equivariant for the conjugation $T$-action on $d T$. From (i) we deduce $\operatorname{Ad}\left(d^{-1}\right)^{*} \mathcal{E}_{T} \cong \mathcal{E}_{T}$. By 28.2 , $\mathcal{E}_{T}$ is $T$-equivariant for the $T$-action on $T$ given by $t_{0}: t \mapsto d^{-1} t_{0} d t t_{0}^{-1}$. Also, $\lambda: d T \rightarrow T, d t \mapsto t$ is compatible with the $T$-action on $T$ (as above) and the $T$ action on $d T$ given by conjugation. Hence $\lambda^{*} \mathcal{E}_{T}$ is $T$-equivariant. Hence $\left.L_{\delta^{-1}}^{*} \mathcal{E}\right|_{d T}$ is $T$-equivariant. We see that (iii) holds.

Conversely, assume that (iii) holds. Then $m^{*} L_{d^{-1}}^{*} \mathcal{E} \cong m^{*} L_{d^{-1}}^{*} \mathcal{E}$ where $m, m^{\prime}$ : $G^{0} \times D \rightarrow D$ are given by $m(g, y)=g y g^{-1}, m^{\prime}(g, y)=y$. Define $j: G^{0} \rightarrow G^{0} \times D$ by $j(g)=(g, d g)$. Then $L_{d^{-1}} m j=\operatorname{Ad}\left(d^{-1}\right), L_{d^{-1}} m^{\prime} j=1$, hence $\operatorname{Ad}\left(d^{-1}\right)^{*} \mathcal{E}=$ $j^{*} m^{*} L_{d^{-1}}^{*} \mathcal{E} \cong j^{*} m^{\prime *} L_{d^{-1}}^{*} \mathcal{E}=\mathcal{E}$. We see that (i) holds.

45.3. Let $\mathcal{E} \in \mathfrak{s}\left(G^{0}\right)$ and let $\mathcal{L}=\mathcal{E}_{T} \in \mathfrak{s}(T)^{1}$. Then $\underline{D} \in \mathbf{W}_{\mathcal{L}}^{\bullet}$. Moreover, for any $w \in \mathbf{W}$ we have $w \in \mathbf{W}_{\mathcal{L}}^{\bullet}$ (see $45.1(\mathrm{iv})$ and 28.3(a)); hence $w \underline{D} \in \mathbf{W}_{\mathcal{L}}^{\bullet}$. Hence the local system $\tilde{\mathcal{L}}$ on $Z_{\emptyset, \mathbf{I}, D}^{w}$ is defined as in 28.7. From the definitions we see that $\tilde{\mathcal{L}}=\pi_{w}^{*} \mathcal{E}_{D}$ where $\pi_{w}: Z_{\emptyset, \mathbf{I}, D}^{w} \rightarrow D$ is the map $\left(B, B^{\prime}, g\right) \mapsto g$. Hence

$$
K_{D}^{w, \mathcal{L}}=\pi_{w !} \pi_{w}^{*} \mathcal{E}_{D}=\mathcal{E}_{D} \otimes \pi_{w !} \pi_{w}^{*} \overline{\mathbf{Q}}_{l}=\mathcal{E}_{D} \otimes \pi_{w !} \overline{\mathbf{Q}}_{l}=\mathcal{E}_{D} \otimes K_{D}^{w} \in \mathcal{D}(D),
$$

(notation of 28.19).

45.4. Now let $\Gamma$ be a closed normal subgroup of $G$ contained in $\mathcal{Z}_{G^{0}}$. Then $G^{\prime}=$ $G / \Gamma$ is a reductive group and the image $D^{\prime}$ of $D$ under the obvious homomorphism $\omega: G \rightarrow G^{\prime}$ is a connected component $D^{\prime}$ of $G^{\prime}$ that generates $G^{\prime}$. We may regard 
naturally $\Gamma$ as a subgroup of the canonical torus $\mathbf{T}$ of $G^{0}$ and we may identify naturally $\mathbf{T} / \Gamma$ with $\mathbf{T}^{\prime}$, the canonical torus of $G^{\prime}$. Let $\mathbf{W}^{\prime}$ be the Weyl group of $G^{\prime 0}$ and let $\mathbf{I}^{\prime}$ be its set of simple reflections (see 26.1). We identify $\mathbf{W}^{\prime}=\mathbf{W}$, $\mathbf{I}^{\prime}=\mathbf{I}$ in an obvious way. Then $\mathbf{W}$ acts on $\mathbf{T}, \mathbf{T}^{\prime}$ compatibly with the canonical map $\mathbf{T} \rightarrow \mathbf{T}^{\prime}$. Let $\omega_{D}: D \rightarrow D^{\prime}$ be the restriction of $\omega$.

Let $w \in \mathbf{W}$. Then $K_{D}^{w}, \bar{K}_{D}^{w} \in \mathcal{D}(D), K_{D^{\prime}}^{w}, \bar{K}_{D^{\prime}}^{w} \in \mathcal{D}\left(D^{\prime}\right)$ are defined. We show

$$
K_{D}^{w} \cong \omega_{D}^{*} K_{D^{\prime}}^{w} \in \mathcal{D}(D), \bar{K}_{D}^{w} \cong \omega_{D}^{*} \bar{K}_{D^{\prime}}^{w} \in \mathcal{D}(D) .
$$

Define $Z_{\emptyset, \mathbf{I}, D^{\prime}}^{w}$ in terms of $G^{\prime}$ in the same way that $Z_{\emptyset, \mathbf{I}, D}^{w}$ is defined in terms of $G$. Let $\pi_{w}: Z_{\emptyset, \mathbf{I}, D}^{w} \rightarrow D$ be as in 45.3 and let $\pi_{w}^{\prime}: Z_{\emptyset, \mathbf{I}, D^{\prime}}^{w} \rightarrow D^{\prime}$ be the analogous map defined in terms of $G^{\prime}$. Define $\omega^{\prime}: Z_{\emptyset, \mathbf{I}, D}^{w} \rightarrow Z_{\emptyset, \mathbf{I}, D^{\prime}}^{w}$ by $\left(B, B^{\prime}, g\right) \mapsto$ $\left(\omega(B), \omega\left(B^{\prime}\right), \omega(g)\right)$. We have a cartesian diagram

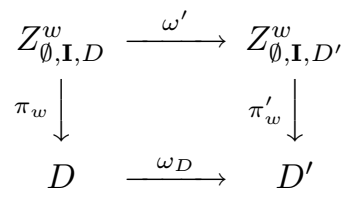

Hence

$$
\omega_{D}^{*} K_{D^{\prime}}^{w}=\omega_{D}^{*} \pi_{w !}^{\prime} \overline{\mathbf{Q}}_{l}=\pi_{w !} \overline{\mathbf{Q}}_{l}=K_{D}^{w}
$$

as required. The second statement in (a) is proved similarly. We set $r=\operatorname{dim}(\Gamma)$. From (a) we deduce for any $i \in \mathbf{Z}$ :

(b) $H^{i}\left(K_{D}^{w}\right) \cong \omega_{D}^{*}\left(H^{i-r}\left(K_{D^{\prime}}^{w}\right)\right)[r], H^{i}\left(\bar{K}_{D}^{w}\right) \cong \omega_{D}^{*}\left(H^{i-r}\left(\bar{K}_{D^{\prime}}^{w}\right)\right)[r]$.

(c) If $A^{\prime} \in \hat{D}^{\prime u n}$, then the perverse sheaf $\omega_{D}^{*}\left(A^{\prime}\right)[r]$ is a direct sum of finitely many objects of $\hat{D}^{u n}$.

45.5. In the setup of 45.4 we assume that $\Gamma=\mathcal{Z}_{G^{0}}^{0}$. Then $\omega_{D}: D \rightarrow D^{\prime}$ is a a fibration with smooth, connected fibres. Using this and 45.4(c) we see that if $A^{\prime} \in \hat{D}^{\prime u n}$ then $\omega_{D}^{*}\left(A^{\prime}\right)[r] \in \hat{D}^{u n}$ and (in the setup of 45.4(b)):

$$
\begin{aligned}
& \left(A^{\prime}: H^{i-r}\left(K_{D^{\prime}}^{w}\right)\right)=\left(\omega_{D}^{*}\left(A^{\prime}\right)[r]: H^{i}\left(K_{D}^{w}\right)\right), \\
& \left(A^{\prime}: H^{i-r}\left(\bar{K}_{D^{\prime}}^{w}\right)\right)=\left(\omega_{D}^{*}\left(A^{\prime}\right)[r]: H^{i}\left(\bar{K}_{D}^{w}\right)\right) .
\end{aligned}
$$

Now let $A \in \hat{D}^{u n}$. We show that $A \cong \omega_{D}^{*}\left(A^{\prime}\right)[r]$ for some $A^{\prime} \in \hat{D}^{\prime u n}$. We can find $w \in \mathbf{W}$ and $i \in \mathbf{Z}$ such that $\left(A: H^{i}\left(K_{D}^{w}\right)\right)>0$. By 45.4(b) we then have $(A$ : $\left.\omega_{D}^{*}\left(H^{i-r}\left(K_{D^{\prime}}^{w}\right)\right)[r]\right)>0$. Hence there exists $A^{\prime} \in \hat{D}^{\prime u n}$ such that $\left(A: \omega_{D}^{*}\left(A^{\prime}\right)[r]\right)>$ 0 , as required. Note that if $A^{\prime}, A^{\prime \prime}$ are objects of $\hat{D}^{\prime u n}$ such that $\omega_{D}^{*}\left(A^{\prime}\right)[r] \cong$ $\omega_{D}^{*}\left(A^{\prime \prime}\right)[r]$, then $A^{\prime} \cong A^{\prime \prime}$ (a standard property of $\omega_{D}^{*}$ ). We see that $A^{\prime} \mapsto \omega_{D}^{*}\left(A^{\prime}\right)[r]$ defines a bijection $\underline{\hat{D}}^{\prime}$ un $\stackrel{\sim}{\longrightarrow} \underline{\hat{D}}^{u n}$.

Let $E \in \operatorname{Irr}(\tilde{\mathbf{W}})$. Let $R_{E} \in \mathcal{K}_{\mathbf{Q}}^{u n}(D)$ be as in $44.6(\mathrm{~b})$ and let $R_{E}^{\prime} \in \mathcal{K}_{\mathbf{Q}}^{u n}\left(D^{\prime}\right)$ be the analogous object defined in terms of $G^{\prime}$. From (a) we see that for $A^{\prime} \in \hat{D}^{\prime \text { un }}$ we have

$$
\left(A^{\prime}: R_{E}^{\prime}\right)=\left(\omega_{D}^{*}\left(A^{\prime}\right)[r]: R_{E}\right) .
$$

Moreover, $\operatorname{since} \operatorname{dim} \operatorname{supp}\left(\omega_{D}^{*} A[r]\right)=\operatorname{dim} \operatorname{supp}(A)+r$, we see from (a) that:

(c) if $D^{\prime}$ has property $\tilde{\mathfrak{A}}$, then $D$ has property $\tilde{\mathfrak{A}}$. 
45.6. In the setup of 45.4 we assume that $\mathcal{Z}_{G^{0}}^{0}=\{1\}$ so that $\Gamma$ is a finite abelian group. Then $\mathcal{Z}_{G^{\prime 0}}^{0}=\{1\}$. Let $\Gamma^{*}=\operatorname{Hom}\left(\Gamma, \overline{\mathbf{Q}}_{l}^{*}\right)$. For $\chi \in \Gamma^{*}$ define ${ }^{D} \chi \in \Gamma^{*}$ by $x \mapsto \chi\left(d x d^{-1}\right)$ (with $d \in N_{D}\left(B^{*}\right) \cap N_{D}(T)$ ). Let ${ }^{D} \Gamma^{*}=\left\{\chi \in \Gamma^{*} ;{ }^{D} \chi=\chi\right\}$. Let $\omega_{0}: G^{0} \rightarrow G^{\prime 0}$ be the restriction of $\omega$. Since $\Gamma$ is abelian, we have

$$
\omega_{0 !} \overline{\mathbf{Q}}_{l} \cong \bigoplus_{\chi \in \Gamma^{*}} \mathcal{E}^{\chi}
$$

where $\mathcal{E}^{\chi}$ is a local system of rank 1 on $G^{\prime 0}$, equivariant for the $G^{0}$-action $g: g^{\prime} \mapsto$ $\omega_{0}(g) g^{\prime}$ of $G^{0}$ on $G^{\prime 0}$, which induces an action of $\Gamma$ on any stalk of $\mathcal{E}^{\chi}$ through $\chi$. Let $\mathcal{E}_{T^{\prime}}^{\chi}$ be the restriction of $\mathcal{E}^{\chi}$ to $T^{\prime}$. Let $\psi^{\prime}$ be the composition $G_{s c}^{0} \stackrel{\psi}{\longrightarrow} G^{0} \stackrel{\omega_{0}}{\longrightarrow} G^{\prime 0}$ ( $\psi$ as in 45.1). For $\chi \in \Gamma^{*}$ we have $\omega_{0}^{*} \mathcal{E}^{\chi} \cong \overline{\mathbf{Q}}_{l}$, hence $\psi^{\prime *} \mathcal{E}^{\chi} \cong \overline{\mathbf{Q}}_{l}$ and $\mathcal{E}^{\chi} \in \mathfrak{s}\left(G^{\prime 0}\right)$. Let $d^{\prime}=\omega(d) \in D^{\prime}$. Define $L_{d^{\prime-1}}^{\prime}: D^{\prime} \stackrel{\sim}{\longrightarrow} G^{\prime 0}$ by $g^{\prime} \mapsto d^{\prime-1} g^{\prime}$. For $\chi \in \Gamma^{*}$ we set $\mathcal{E}_{D^{\prime}}^{\chi}=L_{d^{\prime-1}}^{\prime}{ }^{*} \mathcal{E}^{\chi}$, a local system of rank 1 on $D^{\prime}$. From (a) we deduce

$$
\omega_{D !} \overline{\mathbf{Q}}_{l} \cong \bigoplus_{\chi \in \Gamma^{*}} \mathcal{E}_{D^{\prime}}^{\chi}
$$

It follows that $\bigoplus_{\chi \in \Gamma^{*}} \mathcal{E}_{D^{\prime}}^{\chi}$ is $G^{0}$-equivariant for the $G^{0}$-action

$$
g: g^{\prime} \mapsto \omega_{0}(g) g^{\prime} \omega_{0}(g)^{-1}
$$

on $D^{\prime}$. Hence for any $\chi, \mathcal{E}_{D^{\prime}}^{\chi}$ is $G^{0}$-equivariant for the action (c). Since the restriction of the action (c) to $\Gamma$ is trivial, we see that (c) induces an action of $\Gamma$ on the stalk of $\mathcal{E}_{D^{\prime}}^{\chi}$ at $y \in D^{\prime}$ through a character $\tilde{\chi}$ which is independent of $y$. Moreover, we have $\tilde{\chi}=1$ if and only if $\mathcal{E}_{D^{\prime}}^{\chi}$ is $G^{\prime 0}$-equivariant for the conjugation action of $G^{\prime 0}$ on $D^{\prime}$. By 45.2 (for $G^{\prime}$ instead of $G$ ), this last condition is equivalent to the condition that $\operatorname{Ad}\left(d^{\prime-1}\right)^{*} \mathcal{E}^{\chi} \cong \mathcal{E}^{\chi}$; that is, to the condition that ${ }^{D} \chi=\chi$. Thus we have $\tilde{\chi}=1$ if and only if ${ }^{D} \chi=\chi$. We show:

(d) If $A^{\prime} \in \hat{D}^{\prime}$ and $\chi \in \Gamma^{*}$ satisfies ${ }^{D} \chi \neq \chi$, then the simple perverse sheaf $A_{1}^{\prime}:=\mathcal{E}_{D^{\prime}}^{\chi} \otimes A^{\prime}$ is not in $\hat{D}^{\prime}$.

Indeed, $A^{\prime}$ is a $G^{\prime 0}$-equivariant simple perverse sheaf (for the conjugation action of $G^{\prime 0}$ ) and $A_{1}^{\prime}$ is $G^{0}$-equivariant for the action (c) in such a way that the induced action of $\Gamma$ on stalks is via the nontrivial character $\tilde{\chi}$. We see that $A_{1}^{\prime}$ is not $G^{\prime 0}$-equivariant for the conjugation action of $G^{\prime 0} ;(\mathrm{d})$ follows.

Let $w \in \mathbf{W}$. We show:



Using the cartesian diagram in 45.4 we have

$$
\begin{aligned}
\omega_{D !} K_{D}^{w} & =\omega_{D !} \pi_{w !} \overline{\mathbf{Q}}_{l}=\pi_{w !}^{\prime} \omega_{!}^{\prime} \overline{\mathbf{Q}}_{l}=\pi_{w !}^{\prime} \pi_{w}^{\prime *} \omega_{D !} \overline{\mathbf{Q}}_{l}=\omega_{D !} \overline{\mathbf{Q}}_{l} \otimes\left(\pi_{w !}^{\prime} \pi_{w}^{\prime *} \overline{\mathbf{Q}}_{l}\right) \\
& =\omega_{D !} \overline{\mathbf{Q}}_{l} \otimes \pi_{w !}^{\prime} \overline{\mathbf{Q}}_{l}=\omega_{D !} \overline{\mathbf{Q}}_{l} \otimes K_{D^{\prime}}^{w}=\bigoplus_{\chi \in \Gamma^{*}} \mathcal{E}_{D^{\prime}}^{\chi} \otimes K_{D^{\prime}}^{w} .
\end{aligned}
$$

It remains to use 45.3 (for $G^{\prime}, T^{\prime}$ instead of $G, T$ ).

We show:

(f) If $A^{\prime} \in \hat{D}^{\prime \text { un }}$ and $\chi \in \Gamma^{*},{ }^{D} \chi=\chi, \chi \neq 1$, then $A^{\prime} \notin \hat{D}^{\prime \mathcal{E}_{T^{\prime}}^{\chi}}$.

Indeed, if $A^{\prime} \in \hat{D}^{\prime \mathcal{E}_{T^{\prime}}}$, then by 32.24 there exists $a \in \mathbf{W}$ such that $\overline{\mathbf{Q}}_{l}=a^{*} \overline{\mathbf{Q}}_{l} \cong \mathcal{E}_{T^{\prime}}^{\chi}$ as local systems on $T^{\prime}=\mathbf{T}^{\prime}$. Using $45.1\left(\mathrm{~d}\right.$ ) (for $G^{\prime}$ instead of $G$ ) it follows that $\mathcal{E}^{\chi}=\mathcal{E}^{1}$, hence $\chi=1$, a contradiction. 
We show that for any $A^{\prime} \in \hat{D}^{\prime \text { un }}$ and $i \in \mathbf{Z}$ we have

$$
\left(A^{\prime}: \omega_{D !} H^{i}\left(K_{D}^{w}\right)\right)=\left(A^{\prime}: H^{i}\left(K_{D^{\prime}}^{w}\right)\right) .
$$

We use that $\omega_{D !} H^{i}\left(K_{D}^{w}\right)=H^{i}\left(\omega_{D !} K_{D}^{w}\right)$ which holds since $\omega_{D}$ is a finite covering. Hence the left-hand side of (g) can be rewritten using (e) as

$$
\sum \bigoplus_{\chi \in \Gamma^{*} ; D} \bigoplus_{\chi=\chi}\left(A^{\prime}: H^{i}\left(K_{D^{\prime}}^{w, \mathcal{E}_{T^{\prime}}^{\chi}}\right)\right)+\sum_{\chi \in \Gamma^{*} ; D \chi \neq \chi}\left(A^{\prime}: \mathcal{E}_{D^{\prime}}^{\chi} \otimes H^{i}\left(K_{D^{\prime}}^{w}\right)\right) .
$$

The term corresponding to $\chi$ such that ${ }^{D} \chi \neq \chi$ is 0 by (d); the term corresponding to $\chi$ such that ${ }^{D} \chi=\chi, \chi \neq 1$ is 0 by (f) and (g) follows.

Using 45.4(b) we can reformulate (g) as follows:

$$
\left(A^{\prime}: \omega_{D !} \omega_{D}^{*}\left(H^{i}\left(K_{D^{\prime}}^{w}\right)\right)\right)=\left(A^{\prime}: H^{i}\left(K_{D^{\prime}}^{w}\right)\right) .
$$

In $\mathcal{K}^{\text {un }}\left(D^{\prime}\right)$ we have $H^{i}\left(K_{D^{\prime}}^{w}\right)=\sum_{j=1}^{s} m_{j} A_{j}^{\prime}$ where $A_{1}^{\prime}, A_{2}^{\prime}, \ldots, A_{s}^{\prime}$ are mutually nonisomorphic objects in $\hat{D}^{\prime \text { un }}$ and $m_{j} \in \mathbf{Z}_{>0}$. Applying (h) with $A^{\prime}=A_{h}^{\prime}$ we obtain $\sum_{j=1}^{s} m_{j}\left(A_{h}^{\prime}: \omega_{D !} \omega_{D}^{*}\left(A_{j}^{\prime}\right)\right)=m_{h}$, hence $\sum_{j=1}^{s} m_{j}\left(\omega_{D}^{*}\left(A_{h}^{\prime}\right): \omega_{D}^{*}\left(A_{j}^{\prime}\right)\right)=m_{h}$ for $h \in[1, s]$. Since $\left(\omega_{D}^{*}\left(A_{h}^{\prime}\right): \omega_{D}^{*}\left(A_{j}^{\prime}\right)\right) \geq \delta_{h, j}$ it follows that $\left(\omega_{D}^{*}\left(A_{h}^{\prime}\right): \omega_{D}^{*}\left(A_{j}^{\prime}\right)\right)=$ $\delta_{h, j}$ for $h, j \in[1, s]$. It follows that the perverse sheaf $\omega_{D}^{*} A_{j}^{\prime}$ is simple. Since any $A^{\prime} \in \hat{D}^{\prime \text { un }}$ appears in some $H^{i}\left(K_{D^{\prime}}^{w}\right)$, we see that in our case we have the following refinement of $45.4(\mathrm{c})$ :

(i) if $A^{\prime} \in \hat{D}^{\prime u n}$, then $\omega_{D}^{*}\left(A^{\prime}\right) \in \hat{D}^{u n}$.

Now let $A \in \hat{D}^{u n}$. Let $\omega_{D !}^{0} A$ be the sum of all simple summands of the semisimple perverse sheaf $\omega_{D !} A$ which are in $\hat{D}^{\prime u n}$. We show that:

(j) $\omega_{D !}^{0} A \in \hat{D}^{\prime u n}$.

We can find $w \in \mathbf{W}$ and $i \in \mathbf{Z}$ such that $A$ appears in $H^{i}\left(K_{D}^{w}\right)$. Using 45.4(b) we see that $A$ appears in $\omega_{D}^{*}\left(H^{i}\left(K_{D^{\prime}}^{w}\right)\right)$. Hence there exists $C \in \hat{D}^{\prime u n}$ which appears in $H^{i}\left(K_{D^{\prime}}^{w}\right)$ such that $\left(A: \omega_{D}^{*} C\right)>0$. By (i), $\omega_{D}^{*} C$ is a simple perverse sheaf. It follows that $A \cong \omega_{D}^{*} C$. Thus $C$ appears in $\omega_{D !} A$. In particular, $\omega_{D !}^{0} A \neq 0$. Now assume that $C, C^{\prime}$ are two objects in $\hat{D}^{\prime \text { un }}$ such that both $C$ and $C^{\prime}$ appear in $\omega_{D !} A$. Then $A \cong \omega_{D}^{*} C$; similarly, $A \cong \omega_{D}^{*} C^{\prime}$. Thus the simple objects $\omega_{D}^{*} C, \omega_{D}^{*} C^{\prime}$ are isomorphic. It follows that $\operatorname{dim} \operatorname{Hom}\left(C^{\prime}, \omega_{D !} \omega_{D}^{*} C\right)=1$. We have

$$
\omega_{D !} \omega_{D}^{*} C=C \otimes \omega_{D !} \omega_{D}^{*} \overline{\mathbf{Q}}_{l}=C \otimes \omega_{D !} \overline{\mathbf{Q}}_{l}=\bigoplus_{\chi \in \Gamma^{*}} C \otimes \mathcal{E}_{D^{\prime}}^{\chi} .
$$

It follows that for some $\chi \in \Gamma^{*}$ we have $\operatorname{dim} \operatorname{Hom}\left(C^{\prime}, C \otimes \mathcal{E}_{D}^{\chi}\right)=1$, hence $C^{\prime} \cong$ $C \otimes \mathcal{E}_{D^{\prime}}^{\chi}$. This forces ${ }^{D} \chi=\chi$, by $(\mathrm{d})$. Then $\mathcal{E}_{T^{\prime}}^{\chi}$ is defined and from 45.3 we see that $C \otimes \mathcal{E}_{D^{\prime}}^{\chi} \in \hat{D}^{\prime \mathcal{E}_{T^{\prime}}^{\chi}}$ so that $C^{\prime} \in \hat{D}^{\prime \mathcal{E}_{T^{\prime}}^{\chi}}$. Using (f) we deduce that $\chi=1$ and $C^{\prime} \cong C$. Thus, the semisimple perverse sheaf $\omega_{D !}^{0} A$ is nonzero and isotypic. If $C \in \hat{D}^{\prime \text { un }}$ appears in $\omega_{D !}^{0} A$, then, as we have seen, we have $A \cong \omega_{D}^{*} C$, hence $\operatorname{dim} \operatorname{Hom}\left(C, \omega_{D !} A\right)=1$ so that $\operatorname{dim} \operatorname{Hom}\left(C, \omega_{D !}^{0} A\right)=1$. Thus $\omega_{D !}^{0} A$ is simple. This proves $(\mathrm{j})$.

From (i), (j), and the proof of $(\mathrm{j})$ we see that:

(k) $A^{\prime} \mapsto \omega_{D}^{*}\left(A^{\prime}\right)$ defines a bijection $\underline{\hat{D}}^{\prime}$ un $\stackrel{\sim}{\longrightarrow} \underline{\hat{D}}^{\text {un }}$; the inverse bijection is induced by $A \mapsto \omega_{D !}^{0} A$.

We define $\tilde{\mathbf{W}}^{\prime}$ in terms of $G^{\prime}, D^{\prime}$ in the same way as $\tilde{\mathbf{W}}$ was defined in terms of $G, D$. We may assume that $\tilde{\mathbf{W}}^{\prime}=\tilde{\mathbf{W}}$. Let $E \in \operatorname{Irr}(\tilde{\mathbf{W}})$. Let $R_{E} \in \mathcal{K}_{\mathbf{Q}}^{u n}(D)$ be as 
in 44.6(b) and let $R_{E}^{\prime} \in \mathcal{K}_{\mathbf{Q}}^{u n}\left(D^{\prime}\right)$ be the analogous object defined in terms of $G^{\prime}$. From (g) we see that for $A^{\prime} \in \hat{D}^{\prime \text { un }}$ we have

$$
\left(A^{\prime}: R_{E}^{\prime}\right)=\left(\omega_{D}^{*}\left(A^{\prime}\right): R_{E}\right)
$$

If $A \in \hat{D}^{u n}, w \in \mathbf{W}, i \in \mathbf{Z}$, then

$$
\left(A: H^{i}\left(\bar{K}_{D}^{w}\right)\right)=\left(A: \omega_{D}^{*} H^{i}\left(\bar{K}_{D^{\prime}}^{w}\right)\right)=\left(\omega_{D !} A: H^{i}\left(\bar{K}_{D^{\prime}}^{w}\right)\right)=\left(\omega_{D !}^{0} A: H^{i}\left(\bar{K}_{D^{\prime}}^{w}\right)\right) .
$$

Since $A=\omega_{D}^{*}\left(\omega_{D !}^{0} A\right)$ we have $\operatorname{dim} \operatorname{supp}(A)=\operatorname{dim} \operatorname{supp}\left(\omega_{D !}^{0} A\right)$. We see that

$(\mathrm{m})$ if $D^{\prime}$ has property $\tilde{\mathfrak{A}}$, then $D$ has property $\tilde{\mathfrak{A}}$.

45.7. In the setup of 45.4 assume that $\Gamma=\mathcal{Z}_{G^{0}}$. Then $A^{\prime} \mapsto \omega_{D}^{*}\left(A^{\prime}\right)[r]$ defines a bijection $\underline{\hat{D}}^{\text {un }} \stackrel{\sim}{\longrightarrow} \underline{\hat{D}}^{u n}$. Moreover, for any $w \in \mathbf{W}$, any $A^{\prime} \in \hat{D}^{\prime \text { un }}$ and any $i \in \mathbf{Z}$ we have:

$$
\left(A^{\prime}: H^{i-r}\left(K_{D^{\prime}}^{w}\right)\right)=\left(\omega_{D}^{*}\left(A^{\prime}\right)[r]: H^{i}\left(K_{D}^{w}\right)\right) .
$$

Note that $G / \mathcal{Z}_{G^{0}}$ can be obtained from $G$ in two steps: first we form $G_{1}=G / \mathcal{Z}_{G^{0}}^{0}$ which has $\mathcal{Z}_{G_{1}^{0}}^{0}=\{1\}$ and then we have $G / \mathcal{Z}_{G^{0}}=G_{1} / \mathcal{Z}_{G_{1}^{0}}$. We use 45.5 to compare $G$ to $G_{1}$ and $45.6(\mathrm{k}),(\mathrm{h})$ to compare $G_{1}$ to $G / \mathcal{Z}_{G^{0}}$. The statements above follow.

We define $\tilde{\mathbf{W}}^{\prime}$ in terms of $G^{\prime}, D^{\prime}$ in the same way as $\tilde{\mathbf{W}}$ was defined in terms of $G, D$. We may assume that $\tilde{\mathbf{W}}^{\prime}=\tilde{\mathbf{W}}$. Let $E \in \operatorname{Irr}(\tilde{\mathbf{W}})$. Let $R_{E} \in \mathcal{K}_{\mathbf{Q}}^{u n}(D)$ be as in 44.6(b) and let $R_{E}^{\prime} \in \mathcal{K}_{\mathbf{Q}}^{u n}\left(D^{\prime}\right)$ be the analogous object defined in terms of $G^{\prime}$. From (a) we see that for $A^{\prime} \in \hat{D}^{\prime \text { un }}$ we have

$$
\left(A^{\prime}: R_{E}^{\prime}\right)=\left(\omega_{D}^{*}\left(A^{\prime}\right): R_{E}\right)
$$

Combining $45.5(\mathrm{c}), 45.6(\mathrm{~m})$ we see that:

(c) If $D^{\prime}$ has property $\tilde{\mathfrak{A}}$, then $D$ has property $\tilde{\mathfrak{A}}$.

Now if $A^{\prime} \in \hat{D}^{\prime u n}$, then $A^{\prime}$ is cuspidal if and only if $\omega_{D}^{*}\left(A^{\prime}\right)[r]$ is cuspidal. It follows that:

(d) If $D^{\prime}$ has property $\mathfrak{A}_{0}$, then $D$ has property $\mathfrak{A}_{0}$.

45.8. Assume now that $\mathcal{Z}_{G^{0}}=\{1\}$. Let $\Delta=\mathcal{Z}_{G}$. Let $G^{\prime}=G / \Delta$.

If $g \in G$ satisfies $g g_{1}=g_{1} g \bmod \mathcal{Z}_{G}$ for any $g_{1} \in G$, then for any $g_{1} \in G$ we have $g g_{1} g^{-1} g_{1}^{-1} \in G^{0}$ (since $G / G^{0}$ is abelian), hence $g g_{1} g^{-1} g_{1}^{-1} \in G^{0} \cap \mathcal{Z}_{G} \subset \mathcal{Z}_{G^{0}}=\{1\}$; thus, $g \in \mathcal{Z}_{G}$. We see that $\mathcal{Z}_{G^{\prime}}=\{1\}$.

Let $\pi: G \rightarrow G^{\prime}$ be the obvious map. Then $\pi$ induces an isomorphism $G^{0} \stackrel{\sim}{\longrightarrow} G^{\prime 0}$ and an isomorphism of $D$ onto a connected component $D^{\prime}$ of $G^{\prime}$ which generates $G^{\prime}$. We identify the canonical tori and Weyl groups of $G^{0}, G^{\prime 0}$ in the obvious way.

Let $w \in \mathbf{W}$. From the definitions it is clear that

$$
K_{D}^{w}=\pi^{*} K_{D^{\prime}}^{w}, \bar{K}_{D}^{w}=\pi^{*} \bar{K}_{D^{\prime}}^{w}
$$

It follows that:

(b) $A^{\prime} \mapsto \pi^{*} A^{\prime}$ induces a bijection $\underline{\hat{D}}^{\prime}$ un $\stackrel{\sim}{\longrightarrow} \underline{\hat{D}}^{\text {un }}$. Moreover, if $w \in \mathbf{W}, A^{\prime} \in \hat{D}^{\prime \text { un }}$ and $i \in \mathbf{Z}$, then

$$
\left(A^{\prime}: H^{i}\left(K_{D^{\prime}}^{w}\right)\right)=\left(\pi^{*} A^{\prime}: H^{i}\left(K_{D}^{w}\right)\right) .
$$


Let $E \in \operatorname{Irr}(\tilde{\mathbf{W}})$. Let $R_{E} \in \mathcal{K}_{\mathbf{Q}}^{u n}(D)$ be as in $44.6(\mathrm{~b})$ and let $R_{E}^{\prime} \in \mathcal{K}_{\mathbf{Q}}^{u n}\left(D^{\prime}\right)$ be the analogous object defined in terms of $G^{\prime}$. From (c) we see that for $A^{\prime} \in \hat{D}^{\prime \text { un }}$ we have

$$
\left(A^{\prime}: R_{E}^{\prime}\right)=\left(\pi^{*} A^{\prime}: R_{E}\right)
$$

From the definitions we see that:

(e) If $D^{\prime}$ has property $\tilde{\mathfrak{A}}$, then $D$ has property $\tilde{\mathfrak{A}}$.

(f) If $D^{\prime}$ has property $\mathfrak{A}_{0}$, then $D$ has property $\mathfrak{A}_{0}$.

45.9. Assume now that $\mathcal{Z}_{G}=\{1\}$ with $G^{0}$ adjoint. We have $G^{0}=\prod_{f \in \mathfrak{F}} G_{f}$ where $\mathfrak{F}$ is a finite set and $G_{f}(f \in \mathfrak{F})$ are the maximal connected simple closed subgroups of $G^{0}$. There is a well-defined permutation $\iota: \mathfrak{F} \stackrel{\sim}{\longrightarrow} \mathfrak{F}$ such that $g G_{f} g^{-1}=G_{\iota(f)}$ for all $g \in D, f \in \mathfrak{F}$. Let $\overline{\mathfrak{F}}$ be the set of orbits of $\iota$ on $\mathfrak{F}$. For any $\mathcal{O} \in \overline{\mathfrak{F}}$ we set $G_{\mathcal{O}}=$ $\prod_{f \in \mathcal{O}} G_{f}$. Then $G_{\mathcal{O}}$ is a closed connected normal subgroup of $G$; hence we have a well-defined homomorphism $\theta_{\mathcal{O}}: G \rightarrow \operatorname{Aut}\left(G_{\mathcal{O}}\right)$ given by $g: x \mapsto g x g^{-1}$. The image of $\theta_{\mathcal{O}}$ is denoted by $\tilde{G}_{\mathcal{O}}$. Since $G_{\mathcal{O}}$ is adjoint, $\tilde{G}_{\mathcal{O}}$ is a reductive group with identity component $G_{\mathcal{O}}$; it is generated by its connected component $D_{\mathcal{O}}:=\theta_{\mathcal{O}}(D)$.

Let $\bar{g} \in \mathcal{Z}_{\tilde{G}_{\mathcal{O}}}$. We have $\bar{g}=\theta_{\mathcal{O}}(g)$ with $g \in G$ and $y g x g^{-1} y^{-1}=g y x y^{-1} g^{-1}$ (that is $y^{-1} g^{-1} y g x=x y^{-1} g^{-1} y g$ ) for any $y \in G_{\mathcal{O}}$. Thus $y^{-1} g^{-1} y g$ (an element of $G_{\mathcal{O}}$ ) is in the centre of $G_{\mathcal{O}}$ so that $y^{-1} g^{-1} y g=1$ for any $y \in G_{\mathcal{O}}$. We see that $\theta_{\mathcal{O}}\left(g^{-1}\right)=1$; that is, $\bar{g}^{-1}=1$. Thus, $\mathcal{Z}_{\tilde{G}_{\mathcal{O}}}=\{1\}$.

Note that the homomorphism $G \rightarrow \prod_{\mathcal{O} \in \overline{\mathfrak{F}}} \tilde{G}_{\mathcal{O}}$ given by $\left(\theta_{\mathcal{O}}\right)_{\mathcal{O} \in \overline{\mathfrak{F}}}$ is an imbedding of reductive groups by which we can identify the identity components $G^{0}=$ $\prod_{\mathcal{O} \in \overline{\mathfrak{F}}} G_{\mathcal{O}}$ and the component $D$ with the component $\prod_{\mathcal{O} \in \overline{\mathfrak{F}}} D_{\mathcal{O}}$.

We can identify $\mathbf{W}=\prod_{\mathcal{O} \in \overline{\mathfrak{F}}} \mathbf{W}_{\mathcal{O}}$ where $\mathbf{W}_{\mathcal{O}}$ is the Weyl group of $G_{\mathcal{O}}$. Let $w \in \mathbf{W}$ and let $w_{\mathcal{O}}$ be the $\mathbf{W}_{\mathcal{O}}$-component of $w$. From the definitions we have

$$
K_{D}^{w}=\otimes_{\mathcal{O} \in \overline{\mathfrak{F}}} K_{D_{\mathcal{O}}}^{w_{\mathcal{O}}}, \quad \bar{K}_{D}^{w}=\bigotimes_{\mathcal{O} \in \overline{\mathfrak{F}}} \bar{K}_{D_{\mathcal{O}}}^{w_{\mathcal{O}}}
$$

Hence for $i \in \mathbf{Z}$ we have:

$$
\begin{aligned}
& H^{i}\left(K_{D}^{w}\right)=\bigoplus_{\left(i_{\mathcal{O}}\right) ; \sum_{\mathcal{O}} i_{\mathcal{O}}=i} \otimes_{\mathcal{O} \in \overline{\mathfrak{F}}} H^{i_{\mathcal{O}}}\left(K_{D_{\mathcal{O}}}^{w_{\mathcal{O}}}\right), \\
& H^{i}\left(\bar{K}_{D}^{w}\right)=\bigoplus_{\left(i_{\mathcal{O}}\right) ; \sum_{\mathcal{O}} i_{\mathcal{O}}=i} \otimes_{\mathcal{O} \in \overline{\mathfrak{F}}} H^{i_{\mathcal{O}}}\left(\bar{K}_{D_{\mathcal{O}}}^{w_{\mathcal{O}}}\right) .
\end{aligned}
$$

Assume that $A_{\mathcal{O}} \in \hat{D}_{\mathcal{O}}^{u n}$ is given for each $\mathcal{O} \in \overline{\mathfrak{F}}$. Let $A=\bigotimes_{\mathcal{O} \in \overline{\mathfrak{F}}} A_{\mathcal{O}}$, a simple perverse sheaf on $D$. We can find $w=\left(w_{\mathcal{O}}\right) \in \mathbf{W}$ and $\left(i_{\mathcal{O}}\right) \in \mathbf{N}^{\overline{\mathfrak{F}}}$ such that $\left(A_{\mathcal{O}}: H^{i_{\mathcal{O}}}\left(\bar{K}_{D_{\mathcal{O}}}^{w_{\mathcal{O}}}\right)\right)>0$ for all $\mathcal{O}$, hence $\left(A: \bigotimes_{\mathcal{O} \in \overline{\mathfrak{F}}} H^{i_{\mathcal{O}}}\left(\bar{K}_{D_{\mathcal{O}}}^{w_{\mathcal{O}}}\right)\right)>0$. Using (b) we deduce that $\left(A: H^{i}\left(\bar{K}_{D}^{w}\right)\right)>0$ where $i=\sum_{\mathcal{O}} i_{\mathcal{O}}$. Hence $A \in \hat{D}^{u n}$.

Conversely, let $A \in \hat{D}^{u n}$. We can find $w=\left(w_{\mathcal{O}}\right) \in \mathbf{W}$ and $\left(i_{\mathcal{O}}\right) \in \mathbf{N}^{\overline{\mathfrak{F}}}$ such that $\left(A: H^{i}\left(\bar{K}_{D}^{w}\right)\right)>0$. Using (b) we deduce that $\left(A: \bigotimes_{\mathcal{O} \in \overline{\mathfrak{F}}} H^{i_{\mathcal{O}}}\left(\bar{K}_{D_{\mathcal{O}}}^{w_{\mathcal{O}}}\right)\right)>0$ for some $\left(i_{\mathcal{O}}\right) \in \mathbf{N}^{\overline{\mathfrak{F}}}$ such that $i=\sum_{\mathcal{O}} i_{\mathcal{O}}$. Hence there exist $A_{\mathcal{O}} \in \hat{D}_{\mathcal{O}}^{u n}(\mathcal{O} \in \overline{\mathfrak{F}})$ such that $\left(A_{\mathcal{O}}: H^{i \mathcal{O}}\left(\bar{K}_{D_{\mathcal{O}}}^{w_{\mathcal{O}}}\right)\right)>0$ and $A \cong \nabla_{\mathcal{O} \in \overline{\mathfrak{F}}} A_{\mathcal{O}}$. We see that:

$$
\left(A_{\mathcal{O}}\right) \mapsto \bigotimes_{\mathcal{O} \in \overline{\mathfrak{F}}} A_{\mathcal{O}} \text { induces a bijection } \prod_{\mathcal{O} \in \overline{\mathfrak{F}}} \hat{\underline{D}}_{\mathcal{O}}^{u n} \stackrel{\sim}{\longrightarrow} \underline{\hat{D}}^{u n}
$$


Moreover, if $\left(A_{\mathcal{O}}\right) \leftrightarrow A$ under this bijection, then

$$
\left(A: H^{i}\left(K_{D}^{w}\right)\right)=\sum_{\left(i_{\mathcal{O}}\right) ; \sum_{\mathcal{O}} i_{\mathcal{O}}=i} \prod_{\mathcal{O} \in \overline{\mathfrak{F}}}\left(A_{\mathcal{O}}: H^{i_{\mathcal{O}}}\left(K_{D_{\mathcal{O}}}^{w_{\mathcal{O}}}\right)\right) .
$$

For $\mathcal{O} \in \overline{\mathfrak{F}}$ we define $\tilde{\mathbf{W}}_{\mathcal{O}}, \operatorname{Irr}\left(\tilde{\mathbf{W}}_{\mathcal{O}}\right)$ in terms of $\tilde{G}_{\mathcal{O}}$ in the same way as $\tilde{\mathbf{W}}, \operatorname{Irr}(\tilde{\mathbf{W}})$ were defined in terms of $G$ (see 43.1). For each $\mathcal{O} \in \overline{\mathfrak{F}}$ we assume, given an object, $E_{\mathcal{O}} \in \operatorname{Irr}\left(\tilde{\mathbf{W}}_{\mathcal{O}}\right)$. Then the vector space $E=\bigotimes_{\mathcal{O}} E_{\mathcal{O}}$ can be naturally regarded as an object of $\operatorname{Irr}(\tilde{\mathbf{W}})$. (Any object of $\operatorname{Irr}(\tilde{\mathbf{W}})$ can be obtained in this way.) Define $R_{E_{\mathcal{O}}} \in \mathcal{K}_{\mathbf{Q}}^{u n}\left(D_{\mathcal{O}}\right)$ in terms of $\tilde{G}_{\mathcal{O}}$ in the same way as $R_{E}$ was defined in terms of $G$. Let $\left(A_{\mathcal{O}}\right) \leftrightarrow A$ be as above. From (d) we see that for $A^{\prime} \in \hat{D}^{\prime u n}$ we have

$$
\left(A: R_{E}\right)=\prod_{\mathcal{O} \in \overline{\mathfrak{F}}}\left(A_{\mathcal{O}}: R_{E_{\mathcal{O}}}\right) .
$$

From the definitions we see that:

(f) If $D_{\mathcal{O}}$ has property $\tilde{\mathfrak{A}}$ for any $\mathcal{O}$, then $D$ has property $\tilde{\mathfrak{A}}$.

(g) If $D_{\mathcal{O}}$ has property $\mathfrak{A}_{0}$ for any $\mathcal{O}$, then $D$ has property $\mathfrak{A}_{0}$.

45.10. Let $x, x^{\prime}, y \in \mathbf{W}$ be such that $x^{\prime}=y x \epsilon(y)^{-1}$. We show

$$
g r_{1}\left(K_{D}^{x}\right)=g r_{1}\left(K_{D}^{x^{\prime}}\right) \in \mathcal{K}^{u n}(D) .
$$

The proof is similar to that in [DL, 1.6]. Arguing by induction on $l(y)$ we see that we may assume that $y=s \in \mathbf{I}$.

Assume first that $l(x)=l\left(x^{\prime}\right)=l(s x)+1$. Define an isomorphism $Z_{\emptyset, \mathbf{I}, D}^{x} \rightarrow$ $Z_{\emptyset, \mathbf{I}, D}^{x^{\prime}}$ by $\left(B, B^{\prime}, g\right) \mapsto\left(B_{1}, B_{1}^{\prime}, g\right)$ where $B_{1}, B_{1}^{\prime} \in \mathcal{B}$ are given by $\operatorname{pos}\left(B, B_{1}\right)=s$, $\operatorname{pos}\left(B_{1}, B^{\prime}\right)=s x, B_{1}^{\prime}=g B_{1} g^{-1}$. (We then have $\operatorname{pos}\left(B_{1}, B_{1}^{\prime}\right)=(s x) \epsilon(s)=x^{\prime}$.) It follows that $K_{D}^{x}=K_{D}^{x^{\prime}}$.

The case where $l(x)=l\left(x^{\prime}\right)=l\left(s x^{\prime}\right)+1$ can be reduced to the previous case by exchanging $x, x^{\prime}$.

Assume next that $l\left(x^{\prime}\right)=l(x)+2$. If $\left(B, B^{\prime}, g\right) \in Z_{\emptyset, \mathbf{I}, D}^{x^{\prime}}$, then there are welldefined $B_{1}, B_{1}^{\prime}$ in $\mathcal{B}$ such that $\operatorname{pos}\left(B, B_{1}\right)=s, \operatorname{pos}\left(B_{1}, B_{1}^{\prime}\right)=x, \operatorname{pos}\left(B_{1}^{\prime}, B^{\prime}\right)=$ $\epsilon(s)$. We partition $Z_{\emptyset, \mathbf{I}, D}^{x^{\prime}}$ into two pieces $Z^{\prime}, Z^{\prime \prime}$ (one closed, one open) defined, respectively, by the conditions $B_{1}^{\prime}=g B_{1} g^{-1}, B_{1}^{\prime} \neq g B_{1} g^{-1}$. Let $K^{\prime}, K^{\prime \prime}$ be the direct image with compact support of $\overline{\mathbf{Q}}_{l}$ under the maps $Z^{\prime} \rightarrow D, Z^{\prime \prime} \rightarrow D$, $\left(B, B^{\prime}, g\right) \mapsto g$. Then $g r_{1}\left(K_{D}^{x^{\prime}}\right)=g r_{1}\left(K^{\prime}\right)+g r_{1}\left(K^{\prime \prime}\right)$. Now $\left(B, B^{\prime}, g\right) \mapsto\left(B_{1}, B_{1}^{\prime}, g\right)$ defines an affine line bundle $Z^{\prime} \rightarrow Z_{\emptyset, \mathbf{I}, D}^{x}$. Hence $g r_{1}\left(K^{\prime}\right)=g r_{1}\left(K_{D}^{x}\right)$. It remains to show that $g r_{1}\left(K^{\prime \prime}\right)=0$. Let $\tilde{Z}$ be the set of all $\left(B, B_{0}, B_{0}^{\prime}, B^{\prime}, g\right)$ in $\mathcal{B}^{4} \times D$ such that $\operatorname{pos}\left(B, B_{0}\right)=s, \operatorname{pos}\left(B_{0}, B_{0}^{\prime}\right)=x \epsilon(s), g B g^{-1}=B^{\prime}, g B_{0} g^{-1}=B_{0}^{\prime}$. If $\left(B, B_{0}, B_{0}^{\prime}, B^{\prime}, g\right) \in \tilde{Z}$, there is a unique $\tilde{B} \in \mathcal{B}$ such that $\operatorname{pos}\left(B_{0}, \tilde{B}\right)=x$, $\operatorname{pos}\left(\tilde{B}, B_{0}^{\prime}\right)=\epsilon(s)$. We partition $\tilde{Z}$ into two subsets $\tilde{Z}_{1}, \tilde{Z}_{2}$ (one closed, one open) defined, respectively, by the conditions $\tilde{B}=B^{\prime}, \tilde{B} \neq B^{\prime}$. Let $\tilde{K}, K_{1}, K_{2}$ be the direct image with compact support of $\overline{\mathbf{Q}}_{l}$ under the maps $\tilde{Z} \rightarrow D, \tilde{Z}_{1} \rightarrow D$, $\tilde{Z}_{2} \rightarrow D,\left(B, B_{0}, B_{0}^{\prime}, B^{\prime}, g\right) \mapsto g$. We have $g r_{1}(\tilde{K})=g r_{1}\left(K_{1}\right)+g r_{1}\left(K_{2}\right)$. Now $\left(B, B_{0}, B_{0}^{\prime}, B^{\prime}, g\right) \mapsto\left(B_{0}, B_{0}^{\prime}, g\right)$ is an isomorphism $\tilde{Z}_{1} \rightarrow Z_{\emptyset, \mathbf{I}, D}^{x \epsilon(s)}$ and an affine line bundle $\tilde{Z} \rightarrow Z_{\emptyset, \mathbf{I}, D}^{x \epsilon(s)}$; hence $\tilde{K}=K_{1}$ and $g r_{1}\left(K_{2}\right)=0$. Moreover, $\left(B, B_{0}, B_{0}^{\prime}, B^{\prime}, g\right) \mapsto$ $\left(B, B^{\prime}, g\right)$ is an isomorphism $\tilde{Z}_{2} \rightarrow Z^{\prime \prime}$. Hence $K_{2}=K^{\prime \prime}$ and $g r_{1}\left(K^{\prime \prime}\right)=0$, as required. 
The case where $l(x)=l\left(x^{\prime}\right)+2$ can be reduced to the previous case by exchanging $x, x^{\prime}$. It remains to consider the case where $l(x)=l\left(x^{\prime}\right)=l(s x)-1=l\left(s x^{\prime}\right)-1$. In this case we have $x=x^{\prime}$ (see [DL, 1.6.4]) and there is nothing to prove.

45.11. Assume now that $\mathcal{Z}_{G}=\{1\}$, that $G^{0}$ is adjoint $\neq\{1\}$ and that $G$ has no closed connected normal subgroups other than $G^{0}$ and $\{1\}$. Let $e$ be a pinning (or épinglage, see 1.6) of $G^{0}$ which projects to $\left(B^{*}, T\right)$ under the map $p$ in 1.6. By the adjointness of $G^{0}$ there is a unique element $d \in D$ such that $\operatorname{Ad}(d): G^{0} \rightarrow G^{0}$ stabilizes $e$ under the action 1.6(i). We have $G^{0}=\prod_{f \in \mathfrak{F}} G_{f}$ as in 45.9. Let $\iota: \mathfrak{F} \rightarrow \mathfrak{F}, \overline{\mathfrak{F}}$ be as in 45.9. If $\mathcal{O} \in \overline{\mathfrak{F}}$, then $G_{\mathcal{O}}$ (as in 45.9) is a closed connected normal subgroup of $G$ other than $\{1\}$, hence it is equal to $G^{0}$. Thus, we have $\mathcal{O}=\mathfrak{F}$ that is, $\iota: \mathfrak{F} \rightarrow \mathfrak{F}$ has a single orbit. Let $k=|\mathfrak{F}|$. We can identify $\mathfrak{F}=\mathbf{Z} / k \mathbf{Z}$ in such a way that $\iota(j)=j+1$ for any $j \in \mathbf{Z} / k \mathbf{Z}$.

For $j \in \mathbf{Z} / k \mathbf{Z}$ let $\mathcal{B}_{j}$ be the variety of Borel subgroups of $G_{j}$. We can identify $\mathcal{B}=\prod_{j \in \mathbf{Z} / k \mathbf{Z}} \mathcal{B}_{j}$ by $B \leftrightarrow\left(B_{0}, B_{1}, \ldots, B_{k-1}\right)$ where $B \in \mathcal{B}, B_{j} \in \mathcal{B}_{j}$ satisfy $B=$ $\prod_{j \in \mathbf{Z} / k \mathbf{Z}} B_{j}$. In particular, we have $B^{*}=\prod_{j \in \mathbf{Z} / k \mathbf{Z}} B_{j}^{*}$ where $B_{j}^{*}$ is a Borel subgroup of $G_{j}$. We also have $T=\prod_{j \in \mathbf{Z} / k \mathbf{Z}} T_{j}$, where $T_{j}$ is a maximal torus of $B_{j}^{*}$. We can

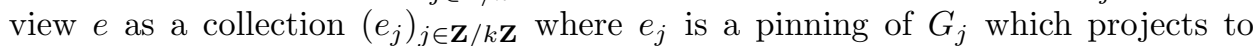
$\left(B_{j}^{*}, T_{j}\right)$. Note that $\operatorname{Ad}(d)$ carries $e_{j}$ to $e_{j+1}$ for any $j \in \mathbf{Z} / k \mathbf{Z}$.

We can identify $\mathbf{W}=\prod_{j \in \mathbf{Z} / k \mathbf{z}} \mathbf{W}_{j}$, where $\mathbf{W}_{j}$ is the Weyl group of $G_{j}$ and $\mathbf{I}=$ $\bigsqcup_{j \in \mathbf{Z} / k \mathbf{Z}} \mathbf{I}_{j}$ where $\mathbf{I}_{j}$ is the set of simple reflections in $\mathbf{W}_{j}$. Recall that $\epsilon: \mathbf{W} \rightarrow \mathbf{W}$ is the automorphism induced by $\operatorname{Ad}(d): G^{0} \rightarrow G^{0}$. We have $\epsilon\left(\mathbf{W}_{j}\right)=\mathbf{W}_{j+1}$ for $j \in \mathbf{Z} / k \mathbf{Z}$.

Now $d^{k}$ normalizes $G_{0}$ and $\operatorname{Ad}\left(d^{k}\right): G_{0} \rightarrow G_{0}$ stabilizes $e_{0}$. Let $G^{\prime}$ be the subgroup of $G$ generated by $G_{0}$ and $d^{k}$. Since $d$ has finite order, $G^{\prime}$ is closed, $G^{\prime 0}=G_{0}$ and $D^{\prime}=d^{k} G_{0}$ is a connected component of $G^{\prime}$ that generates $G^{\prime}$.

We show that $\mathcal{Z}_{G^{\prime}}=\{1\}$. If $g^{\prime} \in \mathcal{Z}_{G^{\prime}}$, then we have $g^{\prime}=d^{k r} x$ for some $r \in \mathbf{Z}, x \in G_{0}$ and $\operatorname{Ad}\left(g^{\prime}\right): G_{0} \rightarrow G_{0}$ is the identity map, hence $\operatorname{Ad}\left(g^{\prime}\right)$ stabilizes $e_{0}$. Since $\operatorname{Ad}\left(d^{k r}\right)$ also stabilizes $e_{0}$, we see that $\operatorname{Ad}(x)$ stabilizes $e_{0}$. Since $G_{0}$ is adjoint, we must have $x=1$, hence $g^{\prime}=d^{k r}$. Thus $g^{\prime}$ commutes with $d$. Since $g^{\prime}$ also centralizes $G_{0}$ and $d, G_{0}$ generate $G$, we see that $g^{\prime}$ centralizes $G$, hence $g^{\prime}=1$ (by our assumption that $\mathcal{Z}_{G}=\{1\}$ ). This verifies our assertion.

Define $\beta: D \rightarrow D^{\prime}$ by $\beta\left(d g_{0} g_{1} \ldots g_{k-1}\right)=d g_{k-1} d g_{k-2} \ldots d g_{0}$ where $g_{j} \in G_{j}$ or equivalently by the requirement that $\zeta^{k} \in \beta(\zeta) G_{1} G_{2} \ldots G_{k-1}$ for $\zeta \in D$. This is a principal $\{1\} \times G_{1} \times G_{2} \times \cdots \times G_{k-1}$-bundle where this group acts on $D$ by restriction of the conjugation action of $G^{0}$. Moreover, $\beta$ is compatible with the conjugation action of $G^{0}$ on $D$ and the conjugation action of $G_{0}$ on $D^{\prime}$ via the homomorphism $G^{0} \rightarrow G_{0}$ which takes $g_{0}$ to $g_{0}$ if $g_{0} \in G_{0}$ and $g_{i}$ to 1 if $i \in[1, k-1]$. We see that (setting $\left.t=(k-1) \operatorname{dim} G_{0}\right)$ :

(a) $A^{\prime} \mapsto \beta^{*} A^{\prime}[t]$ is an equivalence between the category of $G_{0}$-equivariant perverse sheaves on $D^{\prime}$ and the category of $G^{0}$-equivariant perverse sheaves on $D$.

Let $w \in \mathbf{W}_{0} \subset \mathbf{W}$. The variety $Z_{\emptyset, \mathbf{I}, D}^{w}$ may be identified with

$$
\begin{gathered}
\left\{\left(\left(B_{0}, B_{1}, \ldots, B_{k-1}\right),\left(B_{0}^{\prime}, B_{1}^{\prime}, \ldots, B_{k-1}^{\prime}\right), d g_{0} g_{1} \ldots g_{k-1}\right) ; B_{j}, B_{j}^{\prime} \in \mathcal{B}_{j}, g_{j} \in G_{j},\right. \\
\left.B_{j}^{\prime}=\operatorname{Ad}\left(d g_{j-1}\right) B_{j-1}(j \in \mathbf{Z} / k \mathbf{Z}), \operatorname{pos}\left(B_{0}, B_{0}^{\prime}\right)=w, B_{j}=B_{j}^{\prime}(j \neq 0)\right\}
\end{gathered}
$$


or with

$$
\begin{aligned}
& \left\{\left(B_{0}, B_{0}^{\prime}, d g_{0} g_{1} \ldots g_{k-1}\right) ;\right. \\
& \left.B_{0}, B_{0}^{\prime} \in \mathcal{B}_{0}, g_{j} \in G_{j}, B_{0}^{\prime}=\operatorname{Ad}\left(d g_{k-1} d g_{k-2} \ldots d g_{0}\right) B_{0}, \operatorname{pos}\left(B_{0}, B_{0}^{\prime}\right)=w\right\} .
\end{aligned}
$$

We see that we have a cartesian diagram

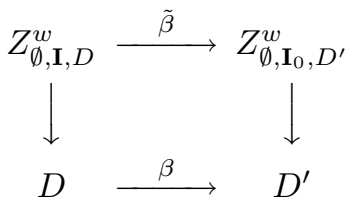

where

$$
\begin{aligned}
& \tilde{\beta}:\left(\left(B_{0}, B_{1}, \ldots, B_{k-1}\right),\left(B_{0}^{\prime}, B_{1}^{\prime}, \ldots, B_{k-1}^{\prime}\right), d g_{0} g_{1} \ldots g_{k-1}\right) \\
& \mapsto\left(B_{0}, B_{0}^{\prime}, d g_{k-1} d g_{k-2} \ldots d g_{0}\right) .
\end{aligned}
$$

Using this cartesian diagram we see that $K_{D}^{w}=\beta^{*} K_{D^{\prime}}^{w}$. Similarly, we have $\bar{K}_{D}^{w}=$ $\beta^{*} \bar{K}_{D^{\prime}}^{w}$. Since $\beta$ is smooth with connected fibres we see that for any $i \in \mathbf{Z}$ we have

$$
H^{i}\left(K_{D}^{w}\right)=\beta^{*} H^{i-t}\left(K_{D^{\prime}}^{w}\right)[t], H^{i}\left(\bar{K}_{D}^{w}\right)=\beta^{*} H^{i-t}\left(\bar{K}_{D^{\prime}}^{w}\right)[t]
$$

and

$$
\begin{aligned}
& \left(\beta^{*} A^{\prime}[t]: H^{i}\left(K_{D}^{w}\right)\right)=\left(A^{\prime}: H^{i-t}\left(K_{D^{\prime}}^{w}\right)\right), \\
& \left(\beta^{*} A^{\prime}[t]: H^{i}\left(\bar{K}_{D}^{w}\right)\right)=\left(A^{\prime}: H^{i-t}\left(\bar{K}_{D^{\prime}}^{w}\right)\right)
\end{aligned}
$$

for any simple perverse sheaf $A^{\prime}$ on $D^{\prime}$. From (b) we see that, if $A^{\prime} \in \hat{D}^{\prime u n}$, then $\beta^{*} A^{\prime}[t] \in \hat{D}^{u n}$.

Conversely, assume that $A \in \hat{D}^{u n}$. Let $\mathcal{X}$ be the set of sequences $\mathbf{s}=\left(s_{1}, s_{2}, \ldots, s_{r}\right)$ in $\mathbf{I}$ such that $\left(A: H^{i}\left(K_{D}^{\mathbf{s}}\right)\right)>0$ for some $i$. Let $\mathcal{X}_{0}$ be the set of all $\mathbf{s}=\left(s_{1}, s_{2}, \ldots, s_{r}\right) \in \mathcal{X}$ such that $s_{h} \in \mathbf{I}_{0}$ for all $h$. Note that $\mathcal{X} \neq \emptyset$. Let $N$ be the minimum value of $N_{\mathbf{s}}:=\sum_{j \in[0, k-1], h \in[1, r] ; s_{h} \in \mathbf{I}_{j}} j$ where $\mathbf{s}=\left(s_{1}, s_{2}, \ldots, s_{r}\right)$ runs through $\mathcal{X}$.

Assume that $N>0$. We choose $\mathbf{s} \in \mathcal{X}$ such that $N_{\mathbf{s}}=N$. We can find $h \in[1, r]$ such that $s_{h} \in \mathbf{I}_{j}$ for some $j \in[1, k-1]$; moreover, we can assume that $h$ is maximum possible with this property. Then $s_{h^{\prime}} \in \mathbf{I}_{0}$ for $h^{\prime} \in[h+1, r]$. Let $\mathbf{s}^{\prime}=\left(s_{1}, s_{2}, \ldots, s_{h-1}, s_{h+1}, \ldots, s_{r}, s_{h}\right)$. Since $s_{h} s_{h^{\prime}}=s_{h^{\prime}} s_{h}$ for $h^{\prime} \in[h+1, r]$, we see using the definitions that $K_{D}^{\mathbf{s}}=K_{D}^{\mathbf{s}^{\prime}}$. Thus $\mathbf{s}^{\prime} \in \mathcal{X}$. Note that $N_{\mathbf{s}^{\prime}}=N$. Let $\mathbf{s}^{\prime \prime}=\left(\epsilon^{-1}\left(s_{h}\right), s_{1}, s_{2}, \ldots, s_{h-1}, s_{h+1}, \ldots, s_{r}\right)$. By 28.16 we have $K_{D}^{\mathbf{s}^{\prime}}=K_{D}^{\mathbf{s}^{\prime \prime}}$. Thus $\mathbf{s}^{\prime \prime} \in \mathcal{X}$. Since $s_{h} \in \mathbf{I}_{j}$ with $j \in[1, k-1]$ we have $\epsilon^{-1}\left(s_{h}\right) \in \mathbf{I}_{j-1}$. Thus $N_{\mathbf{s}^{\prime \prime}}=N_{\mathbf{s}^{\prime}}-1=N-1$. This contradicts the minimality of $N$. We have shown that $N=0$. We choose $\mathbf{s} \in \mathcal{X}$ such that $N_{\mathbf{s}}=0$. We then have $\mathbf{s} \in \mathcal{X}_{0}$. Thus we have $\mathcal{X}_{0} \neq \emptyset$.

By the proof of the implication (iii) $\Longrightarrow$ (i) in 28.13 we deduce that there exists $w \in \mathbf{W}_{0}$ and $i \in \mathbf{Z}$ such that $\left(A: H^{i}\left(K_{D}^{w}\right)\right)>0$. Using (a) we can write $A=\beta^{*} A^{\prime}[t]$ where $A^{\prime}$ is a well-defined simple $G_{0^{-}}$equivariant perverse sheaf on $D^{\prime}$. Using (b) we see that $\left(A^{\prime}: H^{i-t}\left(K_{D^{\prime}}^{w}\right)\right)>0$. Hence $A^{\prime} \in \hat{D}^{\prime u n}$. Thus:

(c) $A^{\prime} \mapsto \beta^{*} A^{\prime}[t]$ induces a bijection $\hat{D}^{\prime u n} \stackrel{\sim}{\longrightarrow} \hat{D}^{u n}$.

We define $\tilde{\mathbf{W}}^{\prime}$ in terms of $G^{\prime}, D^{\prime}$ in the same way as $\tilde{\mathbf{W}}$ was defined in terms of $G, D$; let $\varpi^{\prime}$ be the element of $\tilde{\mathbf{W}}^{\prime}$ which plays the same role for $\tilde{\mathbf{W}}^{\prime}$ as $\varpi$ for $\tilde{\mathbf{W}}$. We can assume that the order of $\varpi^{\prime}$ in $\tilde{\mathbf{W}}^{\prime}$ is the same as the order of $\varpi$ in $\tilde{\mathbf{W}}$. 
Let $E^{\prime} \in \operatorname{Irr}\left(\tilde{\mathbf{W}}^{\prime}\right)$. Then the vector space $E=E^{\prime} \otimes E^{\prime} \otimes \cdots \otimes E^{\prime}$ ( $k$ factors) can be regarded as an object of $\operatorname{Irr}(\tilde{\mathbf{W}})$ with $x=\left(x_{0}, x_{1}, \ldots, x_{k-1}\right)\left(x_{j} \in \mathbf{W}_{j}\right)$ acting by

$$
e_{0}^{\prime} \otimes e_{1}^{\prime} \otimes \cdots \otimes e_{k-1}^{\prime} \mapsto x_{0}\left(e_{0}^{\prime}\right) \otimes \epsilon^{-1}\left(x_{1}\right)\left(e_{1}^{\prime}\right) \otimes \cdots \otimes \epsilon^{-k+1}\left(x_{k-1}\right)\left(e_{k-1}^{\prime}\right)
$$

and $\varpi$ acting by $e_{0}^{\prime} \otimes e_{1}^{\prime} \otimes \cdots \otimes e_{k-1}^{\prime} \mapsto \varpi^{\prime}\left(e_{k-1}^{\prime}\right) \otimes e_{0}^{\prime} \otimes \cdots \otimes e_{k-2}^{\prime}$. (Note that any object of $\operatorname{Irr}(\tilde{\mathbf{W}})$ can be obtained in this way.) Define $R_{E^{\prime}} \in \mathcal{K}_{\mathbf{Q}}^{u n}\left(D^{\prime}\right)$ in terms of $G^{\prime}$ in the same way as $R_{E}$ was defined in terms of $G$. We show that for $A^{\prime} \in \hat{D}^{\prime \text { un }}$ we have

$$
\left(\beta^{*} A^{\prime}[t]: R_{E}\right)=\left(A^{\prime}: R_{E^{\prime}}\right) .
$$

Let $A=\beta^{*} A^{\prime}[t]$. Using (b) we see that the right-hand side of (d) equals

$$
\begin{aligned}
& \left|\mathbf{W}_{0}\right|^{-1} \sum_{x \in \mathbf{W}_{0}, i \in \mathbf{Z}}(-1)^{\operatorname{dim} G^{\prime}+i} \operatorname{tr}\left(x \varpi^{\prime}, E^{\prime}\right)\left(A^{\prime}: H^{i}\left(K_{D^{\prime}}^{x}\right)\right) \\
& =\left|\mathbf{W}_{0}\right|^{-1} \sum_{x \in \mathbf{W}_{0}, i \in \mathbf{Z}}(-1)^{\operatorname{dim} G+i} \operatorname{tr}\left(x \varpi^{\prime}, E^{\prime}\right)\left(A: H^{i}\left(K_{D}^{x}\right)\right) \\
& =\left|\mathbf{W}_{0}\right|^{-1} \sum_{x \in \mathbf{W}_{0}, i \in \mathbf{Z}}(-1)^{\operatorname{dim} G+i} \operatorname{tr}(x \varpi, E)\left(A: H^{i}\left(K_{D}^{x}\right)\right) .
\end{aligned}
$$

(We have used that $\operatorname{tr}(x \varpi, E)=\operatorname{tr}\left(x \varpi^{\prime}, E^{\prime}\right)$ for $x \in \mathbf{W}_{0}$, which follows from the definitions.) Let $\mathbf{W}_{*}=\prod_{j \in \mathbf{Z} / k \mathbf{Z} ; j \neq 0} \mathbf{W}_{j}$. We note that the map $\mathbf{W}_{*} \times \mathbf{W}_{0} \rightarrow \mathbf{W}$, $(y, x) \mapsto y x \epsilon(y)^{-1}$ is a bijection. Using 45.10 (a) we see that the left-hand side of (d) equals

$$
\begin{aligned}
& |\mathbf{W}|^{-1} \sum_{y \in \mathbf{W}_{*}, x \in \mathbf{W}_{0}, i \in \mathbf{Z}}(-1)^{\operatorname{dim} G+i} \operatorname{tr}\left(y x \epsilon(y)^{-1} \varpi, E\right)\left(A: H^{i}\left(K_{D}^{y x \epsilon\left(y^{-1}\right)}\right)\right) \\
& =|\mathbf{W}|^{-1} \sum_{y \in \mathbf{W}_{*}, x \in \mathbf{W}_{0}, i \in \mathbf{Z}}(-1)^{\operatorname{dim} G+i} \operatorname{tr}(x \varpi, E)\left(A: H^{i}\left(K_{D}^{x}\right)\right) .
\end{aligned}
$$

Thus the two sides of (d) are equal.

Using (b) and the definitions we see that:

(e) If $D^{\prime}$ has property $\tilde{\mathfrak{A}}$, then $D$ has property $\tilde{\mathfrak{A}}$.

Note that if $O$ is a $G^{0}$-orbit on $D$, then $\beta(O)$ is a $G^{\prime 0}$-orbit on $D^{\prime}$. Moreover, if $O^{\prime}$ is a $G^{\prime 0}$-orbit on $D^{\prime}$, then $\beta^{-1}\left(O^{\prime}\right)$ is a $G^{0}$-orbit on $D$. We see that:

(f) The map $O \mapsto \beta(O)$ is a bijection between the set of $G^{0}$-orbits on $D$ and the set of $G^{\prime 0}$-orbits on $D^{\prime}$; the inverse bijection takes a $G^{\prime 0}$-orbit $O^{\prime}$ on $D^{\prime}$ to $\beta^{-1}\left(O^{\prime}\right)$.

We show:

(g) If $D^{\prime}$ has property $\mathfrak{A}_{0}$, then $D$ has property $\mathfrak{A}_{0}$.

Let $A \in \hat{D}^{u n c}$. Then $\operatorname{supp}(A)$ is the closure of a single $G^{0}$-orbit $O$ in $D$. We have $A=\beta^{*} A^{\prime}[t]$ where $A^{\prime} \in \hat{D}^{\prime \text { un }}$. Hence $\operatorname{supp}\left(A^{\prime}\right)=\beta^{-1}(\operatorname{supp}(A))$. From (f) we see that $\operatorname{supp}\left(A^{\prime}\right)$ is the closure of a single $G^{\prime 0}$-orbit $O^{\prime}$ in $D^{\prime}$. Hence $A^{\prime}$ is cuspidal. By the assumption of (g) we see that $A^{\prime}$ is zero outside $O^{\prime}$. Hence $A$ is zero outside $\beta^{-1}\left(O^{\prime}\right)$ which is a single $G^{0}$-orbit necessarily equal to $O$. Thus $D$ has property $\mathfrak{A}_{0}$. 


\section{Classification of Unipotent Character Sheaves}

46.1. Let $p \geq 1$ be the characteristic exponent of $\mathbf{k}$. In this section we extend the results of $[\mathrm{L} 3, \mathrm{IV}, \mathrm{V}]$ on the classification of unipotent character sheaves on $D$ from the case $G=G^{0}$ to the general case.

In the remainder of this subsection we assume that $D=G^{0}$ and that (a) below holds:

(a) If $G^{0}$ has a factor of type $E_{8}$ or $F_{4}$, then $p \neq 2$.

We note that:

(b) Any character sheaf on $D$ is clean.

(c) Any admissible complex (see 6.7) on D is a character sheaf.

This is reduced to the case where $G^{0}$ is almost simple as in [L3, 23.21(V)]. In that case, (b) is proved in [L3, IV, V], assuming in addition that: if $G^{0}$ has a factor $E_{8}$, then $p \neq 3, p \neq 5$; if $G^{0}$ has a factor $E_{7}$ or $F_{4}$, then $p \neq 3$; if $G^{0}$ has a factor $E_{6}$, then $p \neq 2$; if $G^{0}$ has a factor $G_{2}$, then $p \neq 2, p \neq 3$. In the remaining cases an additional argument (given by Shoji [Sh, Sec.5] and Ostrik [Os]) is needed. The fact that (b) implies (c) is proved as in [L3, IV, V].

46.2. Assume that $G^{0}$ is semisimple and that for any proper parabolic subgroup $P$ of $G^{0}$ such that $N_{D} P \neq \emptyset$ the following condition is satisfied: any irreducible cuspidal admissible complex on $N_{D} P / U_{P}$ whose support contains some unipotent element is a character sheaf. Let $A \in \hat{D}^{u n c}$ be such that for some unipotent $G^{0}$-orbit $S$ in $D$ and some irreducible cuspidal local system $\mathcal{E}$ on $S$ we have $A=$ $I C(\bar{S}, \mathcal{E})[\operatorname{dim} S]$ extended by 0 on $D-\bar{S}$. We assume that for any $G^{0}$-orbit $C \subset \bar{S}-S$ there is no irreducible cuspidal local system on $C$. We show:

(a) A is clean.

The proof is along the lines of that of [L3, 7.9(II)]. Assume that $A$ is not clean. Let $C \subset \bar{S}-S$ be a $G^{0}$-orbit of minimum possible dimension such that $\mathcal{H}^{i}(A)$ is nonzero on $C$ for some $i$; let $i_{0}$ be the largest $i$ such that $\mathcal{H}^{i}(A)$ is nonzero on $C$. Let $\mathcal{L}$ be an irreducible local system on $C$ which is a direct summand of $\left.\mathcal{H}^{i_{0}}(A)\right|_{C}$. By our assumption, $\mathcal{L}$ is not a cuspidal local system on $C$. By 8.8, 8.3, 8.2(b) we can find $\left(L^{\prime}, S^{\prime}\right) \in \mathbf{A}$ (see 3.5) such that $S^{\prime}$ contains unipotent elements and an irreducible cuspidal local system $\mathcal{E}^{\prime}$ on $S^{\prime}$ such that, setting $\mathfrak{K}^{\prime}=I C\left(\bar{Y}_{L^{\prime}, S^{\prime}}, \pi_{!} \tilde{\mathcal{E}}^{\prime}\right)$ extended by 0 outside $\bar{Y}_{L^{\prime}, S^{\prime}}\left(\tilde{\mathcal{E}}^{\prime}\right.$ as in 5.6), there exists a direct summand $A_{1}$ of $\mathfrak{K}^{\prime}$ whose restriction to the unipotent variety of $D$ is (up to shift) $I C(\bar{C}, \check{\mathcal{L}})$ extended to the unipotent variety by zero outside $\bar{C}$. Let $\left(L^{\prime \prime}, S^{\prime \prime}\right)=\left(G^{0}, S\right)$. Our assumption implies that $L^{\prime} \neq G^{0}$ so that $L^{\prime}, L^{\prime \prime}$ are not $G^{0}$-conjugate. Hence 23.7 is applicable and yields $H_{c}^{j}\left(D, \mathfrak{K}^{\prime} \otimes A\right)=0$ for any $j$. Hence $H_{c}^{j}\left(D, A_{1} \otimes A\right)=0$ for any $j$. Since $\operatorname{supp}(A) \subset$ $\bar{S}$ we have $\operatorname{supp}\left(A_{1} \otimes A\right) \subset \bar{S}$ so that $H_{c}^{j}\left(D, A_{1} \otimes A\right)=H_{c}^{j}\left(\bar{S}, A_{1} \otimes A\right)$. Since $\operatorname{supp}\left(A_{1}\right) \cap \bar{S} \subset \operatorname{supp}\left(\mathfrak{K}^{\prime}\right) \cap \bar{S} \subset \bar{C}$ we have $H_{c}^{j}\left(\bar{S}, A_{1} \otimes A\right)=H_{c}^{j}\left(\bar{C}, A_{1} \otimes A\right)$. Since $A$ is zero on $\bar{C}-C$ (by the minimality of $C$ ) we have $H_{c}^{j}\left(\bar{C}, A_{1} \otimes A\right)=H_{c}^{j}\left(C, A_{1} \otimes A\right)$. We see that $H_{c}^{j}\left(C, A_{1} \otimes A\right)=0$ for all $j$. Since $\left.A_{1}\right|_{C}$ is $\check{\mathcal{L}}$ up to shift, it follows that $H_{c}^{j}(C, \check{\mathcal{L}} \otimes A)=0$ for all $j$. In particular, we have $H_{c}^{2 b+i_{0}}(C, \check{\mathcal{L}} \otimes A)=0$ where $b=\operatorname{dim} C$. Consider the spectral sequence $E_{2}^{r, s}=H_{c}^{r}\left(C, \mathcal{H}^{s}(A) \otimes \check{\mathcal{L}}\right) \Longrightarrow$ $H_{c}^{r+s}\left(C, A \otimes \check{\mathcal{L}}\right.$ ). Then $E_{2}^{r, s}=0$ if $s>i_{0}$ (by our choice of $i_{0}$ ) or if $r>2 b$. It follows that $E_{2}^{2 b, i_{0}}=E_{3}^{2 b, i_{0}}=\cdots=E_{\infty}^{2 b, i_{0}}$. But $E_{\infty}^{2 b, i_{0}}$ is a subquotient of $H_{c}^{2 b+i_{0}}(C, A \otimes \check{\mathcal{L}})$, hence it is zero. It follows that $0=E_{2}^{2 b, i_{0}}=H_{c}^{2 b}\left(C, \mathcal{H}^{i_{0}}(A) \otimes \check{\mathcal{L}}\right)$. Since $\mathcal{L}$ is a direct summand of $\left.\mathcal{H}^{i_{0}}(A)\right|_{C}$, it follows that $H_{c}^{2 b}(C, \mathcal{L} \otimes \check{\mathcal{L}})=0$. This is a contradiction. This proves (a). 
46.3. In this subsection we assume that $G^{0}$ is almost simple, that $m:=\left|G / G^{0}\right|>1$, and that $\mathcal{Z}_{G} \subset G^{0}$. Let $A \in \hat{D}^{u n c}$. Let $S$ be the stratum of $D$ such that $\operatorname{supp}(A)$ is the closure of $S$. Now $\left.A\right|_{S}$ is (up to shift) an irreducible cuspidal local system $\mathcal{E}$. Note that $m$ is 2 or 3 . Let $s \in G$ be a semisimple element and let $u \in G$ be a unipotent element such that $s u=u s \in S$. Let $G^{\prime}=Z_{G}(s)$. Let $\delta$ be the connected component of $G^{\prime}$ that contains $u$. Let $S^{\prime}$ be the (isolated) stratum of $\delta$ that contains $u$. Let $\mathcal{E}^{\prime}$ be the inverse image of $\mathcal{E}$ under $S^{\prime} \rightarrow S, g \mapsto s g$. Let $A^{\prime}=I C\left(\bar{S}^{\prime}, \mathcal{E}^{\prime}\right)\left[\operatorname{dim} S^{\prime}\right]$ extended by 0 on $\delta-\bar{S}^{\prime}$. By $23.4(\mathrm{c}), A^{\prime}$ is a direct sum of cuspidal admissible complexes $A_{j}^{\prime}$ on $G^{\prime 0}$.

We show:

(a) If $p \neq m$, then $A$ is clean.

By our assumption, the image of $u$ in $G / G^{0}$ is 1 . Thus $u \in Z_{G^{0}}(s)$. Since $Z_{G^{0}}(s) / Z_{G^{0}}(s)^{0}$ has order prime to $p$, we see that $u \in Z_{G^{0}}(s)^{0}$. Hence $\delta=$ $Z_{G^{0}}(s)^{0}=G^{\prime 0}$. By 23.4(a) it is enough to show that each $A_{j}^{\prime}$ is clean with respect to $G^{\prime}$. This follows from $46.1(\mathrm{~b})$,(c) applied to $G^{\prime}, G^{\prime 0}$. (Note that $G^{\prime 0}$ does not have a factor $E_{8}$; it can have a factor $F_{4}$ only if $G^{0}$ is of type $E_{6}$ and $p \neq 2$, in which case 46.1(b),(c) are applicable.) This proves (a).

We show:

(b) Assume that $G^{0}$ is of type $A_{n-1}(n \geq 3)$ or $D_{n}(n \geq 2)$. Assume that $p=$ $m=2$ and that for any proper parabolic subgroup $P$ of $G^{0}$ such that $N_{D} P \neq$ $\emptyset$ the following condition is satisfied: any irreducible cuspidal admissible complex on $N_{D} P / U_{P}$ is a character sheaf on $N_{D} P / U_{P}$. Then $A$ is clean.

In this case the image of $s$ in $G / G^{0}$ is 1 . Hence $s \in G^{0}$ and $u \in D$. There is at most one cuspidal admissible complex on $D$. (See 12.9.) This complex must be isomorphic to $A$. Now the conclusion follows from 46.2(a).

46.4. In this subsection we assume that $G^{0}$ is simple of type $A_{n-1}(n \geq 3)$, that $\left|G / G^{0}\right|=2$, that $\mathcal{Z}_{G}=\{1\}$ and that $D \neq G^{0}$. In this case $\epsilon: \mathbf{W} \rightarrow \mathbf{W}$ is given by $w \mapsto w_{0} w w_{0}^{-1}$. In particular, we have $\operatorname{Irr}^{\epsilon}(\mathbf{W})=\operatorname{Irr}(\mathbf{W})$ (see 43.1). We show:

(a) D has property $\mathfrak{A}$.

(b) D has property $\tilde{\mathfrak{A}}$.

(c) If $p=2$, then any irreducible cuspidal admissible complex on $D$ is in $\hat{D}^{u n c}$.

(d) For any $E_{0} \in \operatorname{Irr}(\mathbf{W})$ there is a unique object $A_{E_{0}} \in \hat{D}^{u n}$ (up to isomorphism) which satisfies $R_{E}=s_{E} A_{E_{0}}$ in $\mathcal{K}_{\mathbf{Q}}^{u n}(D)$ for any $E \in \operatorname{Irr}(\tilde{\mathbf{W}})$ such that $\left.E\right|_{\mathbf{Q}[W]}=E_{0}$ (here $s_{E}= \pm 1$ ); moreover, $E_{0} \mapsto A_{E_{0}}$ is a bijection from the set of isomorphism classes in $\operatorname{Irr}(\mathbf{W})$ to $\underline{\hat{D}}^{\text {un }}$.

We can assume that (a)-(d) hold when $n$ is replaced by $n^{\prime}$ where $3 \leq n^{\prime}<n$. (This assumption is empty if $n=3$.)

Note that if $P$ is a proper parabolic subgroup of $G^{0}$ such that $N_{D} P \neq \emptyset$ and such that (setting $D^{\prime}=N_{D} P / U_{P}$ ) either $\hat{D}^{\prime u n c} \neq \emptyset$ or (if $p=2$ ), there is at least one cuspidal admissible complex on $D^{\prime}$, then $P / U_{P}$ is of type $A_{r}$ or a torus and the induction hypothesis shows that $D^{\prime}$ satisfies property $\mathfrak{A}_{0}$ and (if $p=2$ ) any irreducible cuspidal admissible complex on $D^{\prime}$ is in $\hat{D}^{\prime \text { unc }}$.

Using 46.3(a) (if $p \neq 2$ ) and 46.3(b) (if $p=2$ ) we see that (a) holds.

Now let $E_{0} \in \operatorname{Irr}(\mathbf{W})$. We can extend $E_{0}$ to a $\tilde{\mathbf{W}}$-module $E$ in which $\varpi$ acts as $w_{0} \in \mathbf{W}$. We set $e_{E}=(-1)^{a_{E_{0} \otimes s g n}+l\left(w_{0}\right)}, e_{E}^{\prime}=(-1)^{a_{E_{0}}}$. From [L14, (7.6.6)] we see that there exists $x \in \mathbf{c}_{E_{0}}$ such that $\aleph_{x \varpi}=e_{E} \phi_{E},(-1)^{l(x)-\mathbf{a}(x)}=e_{E} e_{E}^{\prime}$. Using 
44.15(c) (which is applicable in view of (a)) we deduce that $e_{E} R_{E}$ is a $\mathbf{Z}$-linear combination of objects $A \in \hat{D}^{u n}$ such that $\mathbf{e}^{A}=e_{E} e_{E}^{\prime}$. Since $\left(R_{E}: R_{E}\right)=1$ we deduce that $R_{E}=s_{E} A_{E_{0}}$ for a well-defined $A_{E_{0}} \in \hat{D}^{u n}$ and $s_{E}= \pm 1$; moreover, $\mathbf{e}^{A_{E_{0}}}=e_{E} e_{E}^{\prime}$. Since any $A \in \hat{D}^{u n}$ satisfies $\left(A: R_{E}\right) \neq 0$ for some $E$ as above we see that $A=A_{E_{0}}$ for some $E_{0}$. Also, if $E_{0}, E_{0}^{\prime}$ are nonisomorphic objects of $\operatorname{Irr}(\mathbf{W})$ and $E, E^{\prime}$ are the corresponding extension to $\tilde{\mathbf{W}}$, then $\left(R_{E}: R_{E^{\prime}}\right)=0$; hence $\left(A_{E_{0}}: A_{E_{0}^{\prime}}\right)=0$ so that $A_{E_{0}} \neq A_{E_{0}^{\prime}}$. We see that (d) holds.

Let $E_{0}, E$ be as above. For $w \in \mathbf{W}$, we have

$$
\left(A_{E_{0}}: g r_{1}\left(K_{D}^{w}\right)\right)= \pm\left(R_{E}: g r_{1}\left(K_{D}^{w}\right)\right)= \pm \operatorname{tr}(w \varpi, E)= \pm \operatorname{tr}\left(w w_{0}, E_{0}\right)
$$

(see $44.7(\mathrm{p}))$. Hence, by $44.14(\mathrm{a})$, the condition that $A_{E_{0}}$ is cuspidal is that $\operatorname{tr}\left(w w_{0}, E_{0}\right)=0$ whenever $w \in \mathbf{W}$ is not $D$-anisotropic. Now $w \in \mathbf{W}$ is not $D$-anisotropic if and only if $w w_{0}$ has even order. Thus the condition that $A_{E_{0}}$ is cuspidal is that $\operatorname{tr}\left(w^{\prime}, E_{0}\right)=0$ whenever $w^{\prime} \in \mathbf{W}$ has even order. The last condition holds if and only if $n$ is of the form $1+2+\cdots+s$ and $E_{0}$ corresponds to the partition of $n$ with parts $1,2, \ldots, s$. (See [L7, 9.2, 9.3, 9.4].) In this case we have $a_{E_{0}}=a_{E_{0} \otimes \mathrm{sgn}}$, hence $\mathbf{e}^{A_{E_{0}}}=(-1)^{l\left(w_{0}\right)}=(-1)^{\mathbf{I}_{\epsilon}}=(-1)^{\operatorname{codim}\left(\operatorname{supp}\left(A_{0}\right)\right)}$. (For the last equality see $44.8(\mathrm{a})$.) Thus the equality $\mathbf{e}^{A}=(-1)^{\operatorname{codim}(\operatorname{supp}(A))}$ holds for any cuspidal $A \in \hat{D}^{u n}$. The analogous equality holds for noncuspidal $A$ in view of the induction hypothesis and 44.15(a). We see that (b) holds.

Now assume that $p=2$. Let $\mathcal{X}_{1}$ be the set of isomorphism classes of irreducible cuspidal admissible complexes on $D$. Let $\mathcal{X}_{2}$ be the set of isomorphism classes of objects in $\hat{D}^{u n c}$. Using 12.9 we see that $\left|\mathcal{X}_{1}\right|=1$ if $n \in\{3,6,10, \ldots\}$ and $\left|\mathcal{X}_{1}\right|=0$ otherwise. By the arguments above we see that $\left|\mathcal{X}_{2}\right|=1$ if $n \in\{3,6,10, \ldots\}$. Clearly, $\mathcal{X}_{2} \subset \mathcal{X}_{1}$. It follows that $\mathcal{X}_{2}=\mathcal{X}_{1}$. This proves (c).

This completes the inductive proof of (a)-(d).

Let $E_{0}, E, x$ be as above. By 44.17 (d) (which is applicable in view of (a),(b)) we have $\left(A_{E_{0}}: R_{\aleph_{x \varpi}}\right) \in \mathbf{N}$, hence $\left(A_{E_{0}}: e_{E} R_{E}\right) \in \mathbf{N}$, hence $\left(s_{E} R_{E}: e_{E} R_{E}\right) \in \mathbf{N}$, hence $s_{E} e_{E} \in \mathbf{N}$, hence $s_{E}=e_{E}$. Thus we have:

(e) $A_{E_{0}}=e_{E} R_{E}$.

46.5. Assume that $G^{0}$ is semisimple and that $A$ is a cuspidal admissible sheaf on $D$ such that $\operatorname{supp}(A)$ is contained in the unipotent variety of $D$. Assume also that $G^{0}$ is of type $A_{n} \times A_{n} \times \cdots \times A_{n}$ ( $r$ factors, $n=1$ or $\left.n=2\right)$. We show:

(a) $A$ is clean.

By arguments in 12.3-12.6 we are reduced to the case where $G^{0}$ is almost simple and $\mathcal{Z}_{G} \subset G^{0}$. If $G=G^{0}$, the conclusion follows from 46.1. Thus we can assume that $G \neq G^{0}$. As in 12.7 we see that we must have $n=2, p=2,\left|G / G^{0}\right|=2$. By 46.4(c), we have $A \in \hat{D}^{u n c}$; using this and 46.4(a), we see that $A$ is clean. This proves (a).

46.6. In the setup of 46.3 we assume that $G^{0}$ is of type $D_{4}$ and $p=m=3$ or of type $E_{6}$ and $p=m=2$. Let $A \in \hat{D}^{\text {unc }}$. We show:

(a) $A$ is clean.

By 12.9 there is exactly one cuspidal admissible complex on $D$ (say $A^{\prime}$ ) whose support is contained in the variety of unipotent elements in $D$. If $A \cong A^{\prime}$, then $A$ is clean by $46.2(\mathrm{a})$. Hence we may assume that $\operatorname{supp}(A)$ is not contained in the variety of unipotent elements in $D$. In this case $G^{\prime 0}$ is of type $A_{1} \times A_{1} \times A_{1} \times A_{1}$ (if $G$ is of type $D_{4}$ ) and of type $A_{2} \times A_{2} \times A_{2}$ (if $G$ is of type $E_{6}$ ). By 23.4(a) it is 
enough to show that each $A_{j}^{\prime}$ (as in 46.3) is clean with respect to $G^{\prime}$. This follows from 46.5(a) with $r=4, n=1$ or $r=3, n=2$. This proves (a).

46.7. In this subsection we assume that $G^{0}$ is simple of type $D_{4}$, that $\left|G / G^{0}\right|=3$, that $\mathcal{Z}_{G}=\{1\}$, hence $D \neq G^{0}$. We show:

(a) D has property $\mathfrak{A}$.

Note that if $P$ is a proper parabolic subgroup of $G^{0}$ such that $N_{D} P \neq \emptyset$ and such that (setting $D^{\prime}=N_{D} P / U_{P}$ ) we have $\hat{D}^{\prime u n c} \neq \emptyset$, then $P$ is a Borel subgroup so that $D^{\prime}$ satisfies property $\mathfrak{A}_{0}$. Using 46.3(a) (if $p \neq 3$ ) and 46.6(a) (if $p=3$ ) we see that (a) holds.

The objects of $\operatorname{Irr}^{\epsilon}(\mathbf{W})$ can be listed as: $1,4,1^{\prime}, 4^{\prime}, 2,6,8$ (each number represents an object of the corresponding degree; moreover, 1 is the unit representation, $1^{\prime}$ is the sign representation, 4 is the reflection representation, $\left.4^{\prime}=4 \otimes 1^{\prime}\right)$. Each of these objects is naturally defined over $\mathbf{Q}$ and it can be viewed as an object of $\operatorname{Irr}(\tilde{\mathbf{W}})$ which is also defined over $\mathbf{Q}$ with $\varpi^{3}=1$ on it; we denote this object of $\operatorname{Irr}(\tilde{\mathbf{W}})$ in the same way as the corresponding object in $\operatorname{Irr}^{\epsilon}(\mathbf{W})$. From [L14, (7.6.5)] we see that each of the elements

$$
\phi_{1}, \phi_{4}, \phi_{1^{\prime}}, \phi_{4^{\prime}}, \phi_{8}+\phi_{2}, \phi_{8}-\phi_{2}, \phi_{8}+\phi_{6}, \phi_{8}-\phi_{6}
$$

is of the form $\aleph_{x \varpi}$ for some $x \in \mathbf{W}$ such that $l(x)-\mathbf{a}(x)=0 \bmod 2$. From this we deduce using 44.15(c) that each of the elements

$$
R_{1}, R_{4}, R_{1^{\prime}}, R_{4^{\prime}}, R_{8}+R_{2}, R_{8}-R_{2}, R_{8}+R_{6}, R_{8}-R_{6}
$$

is a $\mathbf{Z}$-linear combination of objects $A \in \hat{D}^{u n}$ such that $\mathbf{e}^{A}=1$. Since the elements (b) span over $\mathbf{Q}$ the same vector space as that spanned by the $R_{E}$ with $E \in \operatorname{Irr}(\tilde{\mathbf{W}})$ and since each each $A \in \hat{D}^{u n}$ satisfies $\left(A: R_{E}\right) \neq 0$ for some $E \in \operatorname{Irr}(\tilde{\mathbf{W}})$ we see that each $A \in \hat{D}^{u n}$ has nonzero inner product with some element in (b), hence it satisfies $\mathbf{e}^{A}=1$. If $A \in \hat{D}^{u n c}$, then $\operatorname{codim}(\operatorname{supp}(A))=\left|\mathbf{I}_{\epsilon}\right| \bmod 2$; we have $\left|\mathbf{I}_{\epsilon}\right|=2$, hence $\operatorname{codim}(\operatorname{supp}(A))=0 \bmod 2$. Thus $\mathbf{e}^{A}=(-1)^{\operatorname{codim}(\operatorname{supp}(A))}$ if $A \in \hat{D}^{\text {unc }}$. The analogous equality holds for noncuspidal $A$ in view of 44.15 (a) since it trivially holds on $D^{\prime}$ as above. We see that

(c) D has property $\tilde{\mathfrak{A}}$.

By 44.17(d) (which is applicable in view of (a),(b)), the inner product of any $A \in$ $\hat{D}^{u n}$ with any element in (b) is in $\mathbf{N}$. Since the inner product of any two elements in (b) is known (it is 0,1 or 2 ) we see that there exist mutually nonisomorphic objects

$$
A_{1}, A_{4}, A_{1^{\prime}}, A_{4^{\prime}}, a, b, c, d
$$

of $\hat{D}^{u n}$ such that

$$
\begin{aligned}
& R_{1}=A_{1}, R_{4}=A_{4}, R_{1^{\prime}}=A_{1^{\prime}}, R_{4^{\prime}}=A_{4^{\prime}}, R_{8}+R_{2}=a+b, \\
& R_{8}-R_{2}=c+d, R_{8}+R_{6}=a+c, R_{8}-R_{6}=b+d .
\end{aligned}
$$

The list (d) exhausts the isomorphism classes in $\hat{D}^{u n}$ since any $A \in \hat{D}^{u n}$ has nonzero inner product with some element in (b). Note that $R_{8}=(a+b+c+d) / 2$, $R_{2}=(a+b-c-d) / 2, R_{6}=(a-b+c-d) / 2$. 
46.8. In this subsection we assume that $G^{0}$ is simple of type $E_{6}$, that $\left|G / G^{0}\right|=2$, that $\mathcal{Z}_{G}=\{1\}$, hence $D \neq G^{0}$. We show:

(a) D has property $\mathfrak{A}$.

Note that if $P$ is a proper parabolic subgroup of $G^{0}$ such that $N_{D} P \neq \emptyset$ and such that (setting $D^{\prime}=N_{D} P / U_{P}$ ), there is at least one cuspidal admissible complex on $D^{\prime}$; then $P / U_{P}$ is either of type $A_{5}$ or a torus. (The case where $P / U_{P}$ is of type $D_{4}$ is excluded using 23.4(a) when $p \neq 2$ and $12.9(\mathrm{~b})$ when $p=2$.) In either case $D^{\prime}$ satisfies property $\mathfrak{A}_{0}$. Using $46.3(\mathrm{a})$ (if $p \neq 2$ ) and 46.6(a) (if $p=2$ ) we see that (a) holds.

In our case $\epsilon: \mathbf{W} \rightarrow \mathbf{W}$ is given by $w \mapsto w_{0} w w_{0}^{-1}$. The objects of $\operatorname{Irr}(\mathbf{W})$ (up to isomorphism) can be listed as

$$
\begin{aligned}
& 1_{0}, 6_{1}, 20_{2}, 30_{3}, 15_{3}, \tilde{15}_{3}, 64_{4}, 60_{5}, 81_{6}, 24_{6}, 80_{7}, 60_{7}, 90_{7}, 10_{7}, \\
& 20_{7}, 81_{10}, 60_{11}, 24_{12}, 64_{13}, 30_{15}, 15_{15}, \tilde{15}_{15}, 20_{20}, 6_{25}, 1_{36}
\end{aligned}
$$

where $N_{n}$ or $\tilde{N}_{n}$ denotes an object $E_{0} \in \operatorname{Irr}(\mathbf{W})$ such that $\operatorname{dim} E_{0}=N, a_{E_{0}}=n$. Each object of $\operatorname{Irr}(\mathbf{W})$ can be regarded as an object of $\operatorname{Irr}(\tilde{\mathbf{W}})$ on which $\varpi$ acts as $w_{0}$; this object of $\operatorname{Irr}(\tilde{\mathbf{W}})$ is denoted in the same way as the corresponding object in $\operatorname{Irr}(\mathbf{W})$.

From [L14, 7.10] we see that each of the elements

$$
\begin{aligned}
& \phi_{1_{0}},-\phi_{6_{1}}, \phi_{20_{2}},-\phi_{60_{5}}, \phi_{24_{6}}, \phi_{81_{6}}, \phi_{81_{10}}, \phi_{24_{12}},-\phi_{60_{11}}, \phi_{20_{20}},-\phi_{6_{25}}, \phi_{1_{36}}, \\
& -\phi_{30_{3}}-\phi_{15_{3}},-\phi_{30_{3}}+\phi_{15_{3}},-\phi_{30_{3}}-\phi_{15_{3}},-\phi_{30_{3}}+\phi_{1_{5}}, \\
& -\phi_{30_{15}}-\phi_{15_{15}},-\phi_{30_{15}}+\phi_{15_{15}},-\phi_{30_{15}}-\phi_{15_{15}},-\phi_{30_{15}}+\phi_{15_{15}}, \\
& -\phi_{80_{7}}+\phi_{60_{7}}+\phi_{10_{7}},-\phi_{80_{7}}-\phi_{60_{7}}+\phi_{10_{7}},-2 \phi_{80_{7}}-\phi_{10_{7}}, \\
& -\phi_{80_{7}}+\phi_{60_{7}}+\phi_{90_{7}},-\phi_{80_{7}}-\phi_{60_{7}}+\phi_{90_{7}},-2 \phi_{80_{7}}-\phi_{90_{7}},-\phi_{80_{7}}-\phi_{20_{7}}
\end{aligned}
$$

is of the form $\aleph_{x \varpi}(x \in \mathbf{W}, l(x)=\mathbf{a}(x) \bmod 2)$ and that each of the elements $-\phi_{64_{4}}, \phi_{64_{13}}$ is of the form $\aleph_{x \varpi}(x \in \mathbf{W}, l(x) \neq \mathbf{a}(x) \bmod 2)$. From this we deduce using 44.15 (c) that each of the elements

(b) $R_{1_{0}},-R_{6_{1}}, R_{20_{2}},-R_{60_{5}}, R_{24_{6}}, R_{81_{6}}, R_{81_{10}}, R_{24_{12}},-R_{60_{11}}, R_{20_{20}},-R_{6_{25}}, R_{1_{36}}$,

$-R_{30_{3}}-R_{15_{3}},-R_{30_{3}}+R_{15_{3}},-R_{30_{3}}-R_{\tilde{15}_{3}},-R_{30_{3}}+R_{\tilde{15}_{3}}$,

$-R_{30_{15}}-R_{15_{15}},-R_{30_{15}}+R_{15_{15}},-R_{30_{15}}-R_{\tilde{15}_{15}},-R_{30_{15}}+R_{\tilde{15}_{15}}$,

$-R_{80_{7}}+R_{60_{7}}+R_{10_{7}},-R_{80_{7}}-R_{60_{7}}+R_{10_{7}},-2 R_{80_{7}}-R_{10_{7}}$,

$-R_{80_{7}}+R_{60_{7}}+R_{90_{7}},-R_{80_{7}}-R_{60_{7}}+R_{90_{7}},-2 R_{80_{7}}-R_{90_{7}},-R_{80_{7}}-R_{20_{7}}$

is a $\mathbf{Z}$-linear combination of objects $A \in \hat{D}^{u n}$ such that $\mathbf{e}^{A}=1$ and that each of the elements

$$
\text { (c) }-R_{64_{4}}, R_{64_{13}}
$$

is a $\mathbf{Z}$-linear combination of objects $A \in \hat{D}^{u n}$ such that $\mathbf{e}^{A}=-1$. Since the elements in (c) have self-inner product 1 , we have $R_{64_{4}}= \pm A, R_{64_{13}}= \pm A^{\prime}$ where $A, A^{\prime} \in \hat{D}^{u n}$. Since $\left(R_{64_{4}}: R_{64_{13}}\right)=0$, we see that $A \nsubseteq A^{\prime}$. By $44.8(\mathrm{c})$ we have $\mathbf{d}\left(R_{64_{4}}\right)=R_{64_{13}}$, hence $\mathbf{d}(A)= \pm A^{\prime}$. If $A$ were cuspidal, we would have $\mathbf{d}(A)=A$. Thus $A$ is not cuspidal. Similarly, $A^{\prime}$ is not cuspidal. If $A_{1} \in \hat{D}^{u n c}$, then $A_{1}$ must have nonzero inner product with some $R_{E}$, hence with at least one of the elements in (b),(c). But we have just seen that its inner product with any element in (c) is zero. Thus, $A_{1}$ must have nonzero inner product with at least one of the elements in (b). It follows that $\mathbf{e}^{A_{1}}=1$. We have $\operatorname{codim}\left(\operatorname{supp}\left(A_{1}\right)\right)=\left|\mathbf{I}_{\epsilon}\right| \bmod 2$; moreover $\left|\mathbf{I}_{\epsilon}\right|=4$, hence $\operatorname{codim}\left(\operatorname{supp}\left(A_{1}\right)\right)=0 \bmod 2$. Thus, $\mathbf{e}^{A}=(-1)^{\operatorname{codim}(\operatorname{supp}(A))}$ if $A \in \hat{D}^{u n c}$. The analogous equality holds for noncuspidal $A$ in view of 44.15 (a) since it holds on $D^{\prime}$ as above, by $46.4(\mathrm{~b})$. We see that: 
(d) D has property $\tilde{\mathfrak{A}}$.

By $44.17(\mathrm{~d})$ (which is applicable in view of (a),(d)), the inner product of any $A \in$ $\hat{D}^{u n}$ with any element in (b) or (c) is in $\mathbf{N}$. Since the inner products of any two elements in (b) or (c) are known, we see that there exist mutually nonisomorphic objects

$A_{1_{0}}, A_{6_{1}}, A_{20_{2}}, A_{60_{5}}, A_{24_{6}}, A_{81_{6}}, A_{81_{10}}, A_{24_{12}}, A_{60_{11}}, A_{20_{20}}, A_{6_{25}}, A_{1_{36}}$,

$a_{3}, b_{3}, c_{3}, d_{3}, a_{15}, b_{15}, c_{15}, d_{15}, a, b, c, d, e, f, g, h$

of $\hat{D}^{\text {un }}$ such that

$R_{1_{0}}=A_{1_{0}},-R_{6_{1}}=A_{6_{1}}, R_{20_{2}}=A_{20_{2}},-R_{60_{5}}=A_{60_{5}}, R_{24_{6}}=A_{24_{6}}$,

$R_{81_{6}}=A_{81_{6}}, R_{81_{10}}=A_{81_{10}}, R_{24_{12}}=A_{24_{12}},-R_{30_{3}}-R_{15_{3}}=a_{3}+b_{3}$,

$-R_{30_{3}}+R_{15_{3}}=c_{3}+d_{3},-R_{30_{3}}-R_{\tilde{15}_{3}}=a_{3}+c_{3},-R_{30_{3}}+R_{\tilde{15}_{3}}=b_{3}+d_{3}$,

$-R_{30_{15}}-R_{15_{15}}=a_{15}+b_{15},-R_{30_{15}}+R_{15_{15}}=c_{15}+d_{15},-R_{30_{15}}-R_{\tilde{15}_{15}}=a_{15}+c_{15}$,

$-R_{30_{15}}+R_{\tilde{15}_{15}}=b_{15}+d_{15}$

$-R_{80_{7}}+R_{60_{7}}+R_{10_{7}}=a+b+d,-R_{80_{7}}-R_{60_{7}}+R_{10_{7}}=d+e+f$,

$-2 R_{80_{7}}-R_{10_{7}}=b+c+f+g+h,-R_{80_{7}}+R_{60_{7}}+R_{90_{7}}=a+b+c$,

$-R_{80_{7}}-R_{60_{7}}+R_{90_{7}}=c+e+f,,-2 R_{80_{7}}-R_{90_{7}}=b+d+f+g+h$,

$-R_{80_{7}}-R_{20_{7}}=b+f$.

(We use [L14, 7.7(iii)].) Hence we have

$$
\begin{aligned}
& -R_{80_{7}}=(a+3 b+2 c+2 d+e+3 f+2 g+2 h) / 6, R_{60_{7}}=(a+b-e-f) / 2, \\
& R_{90_{7}}=(a+2 c-d+e-g-h) / 3, R_{10_{7}}=(a-c+2 d+e-g-h) / 3, \\
& -R_{20_{7}}=(a-3 b+2 c+2 d+e-3 f+2 g+2 h) / 6 .
\end{aligned}
$$

46.9. We fix an integer $n \geq 1$. Let $W_{n}$ be the group of all permutations of $\left\{1,2, \ldots, n, n^{\prime}, \ldots, 2^{\prime}, 1^{\prime}\right\}$ which commute with the involution $i \leftrightarrow i^{\prime}$. For each $j \in[1, n-1]$ let $s_{j} \in W_{n}$ be the involution which interchanges $j, j+1$ and also $j^{\prime},(j+1)^{\prime}$ and leaves the other elements unchanged. Let $s_{n} \in W_{n}$ be the permutation which interchanges $n, n^{\prime}$ and leaves the other elements unchanged. Define a homomorphism $\chi: W_{n} \rightarrow\{ \pm 1\}$ by the condition $\chi\left(s_{j}\right)=1$ if $j \in[1, n-1]$, $\chi\left(s_{n}\right)=-1$.

We now assume that $n \geq 2$. Then $W_{n}^{\prime}:=\operatorname{ker} \chi$ is a Coxeter group on the generators $s_{j}(j \in[1, n-1])$ and $s_{n} s_{n-1} s_{n}$.

For $h \in[2, n-1]$ let $W_{n, h}$ be the subgroup of $W_{n}$ consisting of the permutations in $W_{n}$ which carry each of

$$
\begin{gathered}
\{1,2, \ldots, n-h\},\left\{n-h+1, n-h+2, \ldots, n, n^{\prime}, \ldots,(n-h+2)^{\prime},(n-h+1)^{\prime}\right\}, \\
\left\{1^{\prime}, 2^{\prime}, \ldots,(n-h)^{\prime}\right\}
\end{gathered}
$$

into itself. We may identify in an obvious way $W_{n, h}$ with $\mathfrak{S}_{n-h} \times W_{h}$ where $\mathfrak{S}_{n-h}$ is the symmetric group in $n-h$ letters.

46.10. Let $m \in \mathbf{N}$. Let $X_{n}^{m}$ be the set of all ordered pairs $(S, T)$ ("symbols") of distinct subsets of $\mathbf{N}$ (with $|S|=|T|=m$ ) such that

$$
\sum_{x \in S} x+\sum_{x \in T} x=n+m^{2}-m .
$$

We define a "shift" map $X_{n}^{m} \rightarrow X_{n}^{m+1}$ by $(S, T) \mapsto(\{0\} \cup(S+1),\{0\} \cup(T+1))$. Using the shift maps we can form the direct limit $X_{n}=\lim _{m \rightarrow \infty} X_{n}^{m}$. We have an obvious map $X_{n}^{m} \rightarrow X_{n}$. If $m \geq n$, then any $(S, T) \in X_{n}^{m+1}$ satisfies $0 \in S, 0 \in T$. Hence if $m \geq n$, the shift map $X_{n}^{m} \rightarrow X_{n}^{m+1}$ is a bijection. We shall sometimes 
identify $X_{n}$ with $X_{n}^{m}$ with some fixed $m \geq n$. But some elements of $X_{n}$ can be represented by elements of $X_{n}^{m}$ where $m<n$.

Note that if $(S, T) \in X_{n}^{m}$, then $S \cup T \subset[0, n+m-1]$. Thus $X_{n}^{m}$ is finite for any $m$ so that $X_{n}$ is finite.

Let $\bar{X}_{n}^{m}$ be the set of all pairs $(M, N)$ of disjoint subsets of $\mathbf{N}$ such that $M \neq \emptyset$, $|M|+2|N|=2 m$ and

$$
\sum_{x \in M} x+2 \sum_{x \in N} x=n+m^{2}-m
$$

We define a "shift" map $\bar{X}_{n}^{m} \rightarrow \bar{X}_{n}^{m+1}$ by $(M, N) \mapsto(M+1,\{0\} \cup(N+1))$. Using the shift maps we can form the direct limit $\bar{X}_{n}=\lim _{m \rightarrow \infty} \bar{X}_{n}^{m}$. We have an obvious map $\bar{X}_{n}^{m} \rightarrow \bar{X}_{n}$. If $m \geq n$, then any $(M, N) \in \bar{X}_{n}^{m+1}$ satisfies $0 \in N$ (hence $0 \notin M$ ). Hence if $m \geq n$, the shift map $\bar{X}_{n}^{m} \rightarrow \bar{X}_{n}^{m+1}$ is a bijection. We shall sometimes identify $\bar{X}_{n}$ with $\bar{X}_{n}^{m}$ with some fixed $m \geq n$.

For $(M, N) \in \bar{X}_{n}^{m}$ let $\mathcal{V}_{M}$ (resp. $V_{M}$ ) be the set of all subsets of $M$ with cardinal $|M| / 2$ (resp. with even cardinal); we regard $V_{M}$ as an $\mathbf{F}_{2}$-vector space with addition $E, E^{\prime} \mapsto E * E^{\prime}=\left(E \cup E^{\prime}\right)-\left(E \cap E^{\prime}\right)$. Let

$$
V_{M}^{\prime}=\left\{\eta: V_{M} \rightarrow \mathbf{F}_{2} \text {-linear, } \eta(M)=1\right\} ;
$$

here $M$ is viewed as an element of $V_{M}$.

Define $t_{M}: M \rightarrow \mathbf{F}_{2}$ by $t_{M}(x)=\left|\left\{x^{\prime} \in M ; x^{\prime}<x\right\}\right| \bmod 2$. Define an injective map $\mathcal{V}_{M} \rightarrow V_{M}$ by

$$
H \mapsto H^{\sharp}:=t_{M}^{-1}(1) * H ;
$$

the image of this map is denoted by $\tilde{\mathcal{V}}_{M}$.

We define a (surjective) map $\zeta: X_{n}^{m} \rightarrow \bar{X}_{n}^{m}$ by $(S, T) \mapsto(S * T, S \cap T)$; if $(M, N) \in \bar{X}_{n}^{m}$, then $H \mapsto(N \cup H, N \cup(M-H))$ is a bijection $\mathcal{V}_{M} \leftrightarrow \zeta^{-1}(M, N)$.

46.11. An irreducible $\mathbf{Q}\left[W_{n}\right]$-module is said to be nondegenerate if its restriction to $W_{n}^{\prime}$ is irreducible. To a nondegenerate irreducible $\mathbf{Q}\left[W_{n}\right]$-module we associate an element $(S, T)$ of $X_{n}$ as in [L7, 2.7(ii)]. We obtain a bijection $[[S, T]] \leftrightarrow(S, T)$ between the set of nondegenerate irreducible $\mathbf{Q}\left[W_{n}\right]$-modules (up to isomorphism) and $X_{n}$. Note that $[[S, T]]$ and $[[T, S]]$ have the same restriction to $W_{n}^{\prime}$.

46.12. In 46.12-46.24 we assume that $G^{0}$ is adjoint of type $D_{n}(n \geq 2)$, that $\left|G / G^{0}\right|=2$, that $\mathcal{Z}_{G}=\{1\}$, hence $D \neq G^{0}$. We choose an isomorphism of $\mathbf{W}$ with $W_{n}^{\prime}$ as Coxeter groups and we use it to identify the two groups. We define a surjective homomorphism $\tilde{\mathbf{W}} \rightarrow W_{n}$ : it takes $\varpi$ to $s_{n}$ and its restriction to $\mathbf{W}$ is the obvious imbedding $\mathbf{W}=W_{n}^{\prime} \rightarrow W_{n}$. Via this homomorphism any nondegenerate irreducible $\mathbf{Q}\left[W_{n}\right]$-module can be viewed as an object of $\operatorname{Irr}(\tilde{\mathbf{W}})$ so that the set of isomorphism classes of objects of $\operatorname{Irr}(\tilde{\mathbf{W}})$ can be identified with the set of isomorphism classes of nondegenerate irreducible $\mathbf{Q}\left[W_{n}\right]$-modules, hence with the set $\left\{[[S, T]] ;(S, T) \in X_{n}\right\}$. Note that for $(S, T),\left(S^{\prime}, T^{\prime}\right)$ in $X_{n}$ we have $\zeta(S, T)=\zeta\left(S^{\prime}, T^{\prime}\right)$ if and only if the two-sided cells attached to [[S,T]] and to $\left[\left[S^{\prime}, T^{\prime}\right]\right]$ coincide. Thus $\bar{X}_{n}$ may be viewed as as indexing set for the two-sided cells of $\mathbf{W}$ which are $\epsilon$-stable. We write $\mathbf{c}_{M, N}$ for the two-sided cell of $\mathbf{W}$ corresponding to $(M, N) \in \bar{X}_{n}$. 
46.13. For any two-element subset $C$ of $\mathbf{N}$ let $[C]$ be the closed interval in $\mathbf{R}$ with extremities in $C$. Let $M$ be a finite nonempty subset of $\mathbf{N}$ of even cardinal. An admissible arrangement of $M$ is a set $\Phi$ of two-element subsets of $M$ forming a partition of $M$ with the following property: for any four element subset of $M$ of the form $C \sqcup C^{\prime}$ where $C \in \Phi, C^{\prime} \in \Phi$, we have $[C] \subset\left[C^{\prime}\right]$ or $\left[C^{\prime}\right] \subset[C]$ or $[C] \cap\left[C^{\prime}\right]=0$. (This agrees with the definition in [L14, p. 164].) For example, the admissible arrangements of $\{0,1,2,3,4,5\}$ are

$$
\begin{gathered}
\Phi_{1}=\{(0,1),(2,3),(4,5)\}, \Phi_{2}=\{(0,5),(1,2),(3,4)\}, \Phi_{3}=\{(0,3),(1,2),(4,5)\}, \\
\Phi_{4}=\{(0,1),(2,5),(3,4)\}, \Phi_{5}=\{(0,5),(1,4),(2,3)\} .
\end{gathered}
$$

If $\Psi$ is a subset of $\Phi$ and $i \in \mathbf{F}_{2}$, we denote by $\Psi^{i}$ the set of all $x \in t_{M}^{-1}(i)$ such that $x$ belongs to some pair in $\Psi$.

Now let $(M, N) \in \bar{X}_{n}^{m}$. Let $\Phi$ be an admissible arrangement of $M$ and let $\hat{\Phi} \subset \Phi$ be a subset such that $|\hat{\Phi}|$ is odd. We set

$$
c(M, N, \Phi, \hat{\Phi})=\frac{1}{2} \sum_{\Psi \subset \Phi}(-1)^{|\hat{\Phi} \cap \Psi|} \phi_{\left[\left[\Psi^{0} \cup(\Phi-\Psi)^{1} \cup N, \Psi^{1} \cup(\Phi-\Psi)^{0} \cup N\right]\right]} \in \mathcal{R}(\tilde{\mathbf{W}}) .
$$

The last inclusion holds since for any $\Psi \subset \Phi$ we have $(-1)^{|\hat{\Phi} \cap \Psi|}=-(-1)^{|\hat{\Phi} \cap(\Phi-\Psi)|}$. From [L14, (5.18.1)] we see that:

(a) There exists $x \in \mathbf{W}$ such that $c(M, N, \Phi, \hat{\Phi})=\aleph_{x \varpi}$ and $l(x)=\mathbf{a}(x) \bmod 2$.

From [L15, 1.19] we see that

(b) If $H \in \mathcal{V}_{M}$, then there exists an admissible arrangement $\Phi$ of $M$ and $\Psi \subset \Phi$ such that $H=\Psi^{0} \cup(\Phi-\Psi)^{1}$; that is,

$$
[[N \cup H, N \cup(M-H)]]=\left[\left[\Psi^{0} \cup(\Phi-\Psi)^{1} \cup N, \Psi^{1} \cup(\Phi-\Psi)^{0} \cup N\right]\right] ;
$$

moreover,

$$
\phi_{[[N \cup H, N \cup(M-H)]]}=2^{-|M / 2|+1} \sum_{\hat{\Phi} \subset \Phi ;|\hat{\Phi}|=\text { odd }}(-1)^{\left|\hat{\Phi} \cap \Psi^{\prime}\right|} c(M, N, \Phi, \hat{\Phi}) .
$$

46.14. We now state some properties (a)-(d) of $D$.

(a) D has property $\mathfrak{A}$.

(b) D has property $\tilde{\mathfrak{A}}$.

In view of (a),(b), the results in 44.17-44.21 are applicable to $D$. In particular, for any $\epsilon$-stable two-sided cell $\mathbf{c}$ of $\mathbf{W}$, the subcategory $\hat{D}_{\mathbf{c}}^{u n}$ of $\hat{D}^{u n}$ is defined as in 44.19. We shall write $\hat{D}_{M, N}^{u n}, \underline{D}_{M, N}^{u n}$ instead of $\hat{D}_{\mathbf{c}_{M, N}}^{u n}, \underline{\hat{D}}_{\mathbf{c}_{M, N}}^{u n}$ where $(M, N) \in \bar{X}_{n}$.

(c) For any $m \geq n$ and any $(M, N) \in \bar{X}_{n}^{m}$ there exists a bijection $\eta \mapsto A_{\eta}$, $V_{M}^{\prime} \leftrightarrow \underline{D}_{M, N}^{u n}$ such that

$$
\left(A_{\eta}: R_{[[N \sqcup H, N \sqcup(M-H)]]}\right)=2^{-|M| / 2+1}(-1)^{\eta\left(t_{M}^{-1}(1) * H\right)}
$$

for any $\eta \in V_{M}^{\prime}, H \in \mathcal{V}_{M}$.

(d) If $p=2$, then any irreducible cuspidal admissible complex on $D$ is in $\hat{D}^{\text {unc }}$; moreover, $\hat{D}^{\text {unc }}$ is empty unless $n=s^{2}$ with $s$ odd, $s \geq 3$, in which case $\hat{D}^{u n c}$ has exactly one object up to isomorphism; its support is contained in the set of unipotent elements of $D$. 
The proofs for (a)-(d) are given in 46.15-46.23 under the induction hypothesis that (a)-(d) hold when $n$ is replaced by $n^{\prime}$ with $2 \leq n^{\prime}<n$. (This assumption is empty if $n=2$.)

46.15. If $P$ is a proper parabolic subgroup of $G^{0}$ such that $N_{D} P \neq \emptyset$ and such that (setting $D^{\prime}=N_{D} P / U_{P}$ ) either $\hat{D}^{\prime u n c} \neq \emptyset$ or (if $p=2$ ) there is at least one cuspidal admissible complex on $D^{\prime}$, then $P / U_{P}$ is of type $D_{r}$ or a torus and the induction hypothesis shows that $D^{\prime}$ satisfies property $\mathfrak{A}_{0}$ and (if $p=2$ ) any irreducible cuspidal admissible complex on $D^{\prime}$ is in $\hat{D}^{\prime u n c}$.

Using 46.3(a) (if $p \neq 2$ ) and 46.3(b) (if $p=2$ ) we see that 46.14(a) holds.

Using 46.13(a) and 44.15(c) (which is applicable in view of 46.14(a)) we see that for any $M, N, \Phi, \hat{\Phi}$ as in $46.13(\mathrm{a}), R_{c(M, N, \Phi, \hat{\Phi})}$ is a Z-linear combination of objects $A \in \hat{D}^{u n}$ such that $\mathbf{e}^{A}=1$. Using $46.13(\mathrm{~b})$ we deduce that for any $E \in \operatorname{Irr}(\tilde{\mathbf{W}})$, $R_{E}$ is a $\mathbf{Z}$-linear combination of objects $A \in \hat{D}^{u n}$ such that $\mathbf{e}^{A}=1$. Since any $A \in \hat{D}^{u n}$ appears with nonzero coefficient in $R_{E}$ for some $E \in \operatorname{Irr}(\tilde{\mathbf{W}})$, we see that any $A \in \hat{D}^{u n}$ satisfies $\mathbf{e}^{A}=1$.

We show:

(a) If $\hat{D}^{u n c} \neq \emptyset$, then $n$ is odd.

If $p=2$, this follows from 12.9(b). If $p \neq 2$, then we can find an isolated semisimple element $s \in D$ such that $Z_{G}(s)^{0}$ carries a cuspidal admissible complex supported on the unipotent variety of $Z_{G}(s)^{0}$ (see $23.4(\mathrm{~b})$ ). Now $Z_{G}(s)^{0}$ is either semisimple of type $B_{n-1}$ (and then $n-1$ must be even by the known theory for connected classical groups) or is semisimple of type $B_{a} \times B_{b}$ with $a \geq 1, b \geq 1, a+b=n-1$ (and then $a, b$ must be even and $n-1$ must be even). Thus (a) holds.

Now if $A \in \hat{D}^{u n c}$, we have $(-1)^{\operatorname{codim}(\operatorname{supp}(A))}=(-1)^{\left|\mathbf{I}_{\epsilon}\right|}=(-1)^{n-1}$ and this equals 1 by (a). Thus we have $\mathbf{e}^{A}=(-1)^{\operatorname{codim}(\operatorname{supp}(A))}$ for any cuspidal $A \in \hat{D}^{u n}$. The analogous equality holds for noncuspidal $A$ in view of the induction hypothesis and 44.15(a). We see that 46.14(b) holds.

46.16. For $h \in[2, n-1]$ let $P^{h}$ be the parabolic subgroup of $G^{0}$ which contains $B^{*}$ and is such that the Weyl group of $P^{h} / U_{P^{h}}$ is the subgroup of $\mathbf{W}_{I^{h}}:=W_{n}^{\prime} \cap W_{n, h}$ of $\mathbf{W}=W_{n}^{\prime}$. Then $\tilde{\mathbf{W}}_{I^{h}}$ (the subgroup of $\tilde{\mathbf{W}}$ generated by $\mathbf{W}_{I^{h}}$ and $\varpi$, see 43.8 ) is the inverse image under $\tilde{\mathbf{W}} \rightarrow W_{n}$ of $W_{n, h}$ and $\operatorname{Irr}\left(\tilde{\mathbf{W}}_{I^{h}}\right)$ can be identified under $\tilde{\mathbf{W}}_{I^{h}} \rightarrow W_{n, h}$ with the set of isomorphism classes of irreducible $\mathbf{Q}\left[W_{n, h}\right]$-modules of the form $E \otimes E^{\prime}$ where $E$ is an irreducible $\mathbf{Q}\left[\mathfrak{S}_{n-h}\right]$-module and $E^{\prime}$ is an irreducible nondegenerate $\mathbf{Q}\left[W_{h}\right]$-module. Let $G^{h}=N_{G} P^{h} / U_{P^{h}}$. Then $D^{h}=N_{D} P^{h} / U_{P^{h}}$ is a connected component of $G^{h}$. We have $G^{h} / Z^{h}=P G L_{n-h} \times \bar{G}^{h}$ where $Z^{h}$ is a onedimensional torus in the centre of $\left(G^{h}\right)^{0}$ and $\bar{G}^{h}$ is a group like $G$ (with $n$ replaced by $h$ ). Hence 46.14(a)-46.14(d) hold for $D^{h}$ instead of $D$ (by the induction hypothesis) and the objects in $\left(\hat{D}^{h}\right)^{u n}$ can be written in the form $A \otimes A^{\prime}$ with $A \in \widehat{P G L}_{n-h}^{u n}$ and $A^{\prime} \in\left(\hat{\bar{D}}^{h}\right)^{\text {un }}$ (where $\bar{D}^{h}=D^{h} / Z^{h}$ ).

46.17. Using 46.13(a) and 44.17(d) (which is applicable in view of 46.14(a), 46.14(b)) we see that for any $(M, N) \in \bar{X}_{n}^{m}$, any admissible arrangement $\Phi$ of $M$ and any $\hat{\Phi} \subset \Phi$ with $|\hat{\Phi}|=$ odd we have that $R_{c(M, N, \Phi, \hat{\Phi})}$ is a $\mathbf{N}$-linear combination 
of objects in $\hat{D}^{u n}$ or equivalently that

$$
\frac{1}{2} \sum_{\Psi \subset \Phi}(-1)^{|\hat{\Phi} \cap \Psi|} R_{\left[\left[\Psi^{0} \cup(\Phi-\Psi)^{1} \cup N, \Psi^{1} \cup(\Phi-\Psi)^{0} \cup N\right]\right]}
$$

is an N-linear combination of objects in $\hat{D}^{u n}$.

46.18. We prove $46.14(\mathrm{c})$ assuming that $|M|=2$. We have $M=\{x, y\}$ with $x<y$. From 46.17 we see that $R_{[[N \sqcup\{y\}, N \sqcup(\{x\})]]}$ is an $\mathbf{N}$-linear combination of objects in $\hat{D}_{M, N}^{u n}$. Since $R_{[[N \sqcup\{y\}, N \sqcup(\{x\})]]}$ has self-inner product 1 it must be equal to a single object of $\hat{D}_{M, N}^{u n}$ and the desired result follows.

46.19. We prove 46.14(c) assuming that $|M|=4$. We have $M=\{x, y, z, u\}$ with $x<y<z<u$. From 46.17 we see that:

$$
\begin{aligned}
& R_{[[N \sqcup\{y, u\}, N \sqcup(\{x, z\})]]} \pm R_{[[N \sqcup\{x, u\}, N \sqcup(\{y, z\})]]}, \\
& R_{[[N \sqcup\{y, u\}, N \sqcup(\{x, z\})]]} \pm R_{[[N \sqcup\{z, u\}, N \sqcup(\{x, y\})]]}
\end{aligned}
$$

are $\mathbf{N}$-linear combinations of objects in $\hat{D}_{M, N}^{u n}$. Since the inner products of any two elements in (a) are known (they are 0,1 or 2 ) we see that there exist four mutually non-isomorphic objects $a, b, c, d$ of $\hat{D}_{M, N}^{u n}$ such that

$$
\begin{aligned}
& R_{[[N \sqcup\{y, u\}, N \sqcup(\{x, z\})]]}+R_{[[N \sqcup\{x, u\}, N \sqcup(\{y, z\})]]}=a+b, \\
& R_{[[N \sqcup\{y, u\}, N \sqcup(\{x, z\})]]}-R_{[[N \sqcup\{x, u\}, N \sqcup(\{y, z\})]]}=c+d, \\
& R_{[[N \sqcup\{y, u\}, N \sqcup(\{x, z\})]]}+R_{[[N \sqcup\{z, u\}, N \sqcup(\{x, y\})]]}=a+c, \\
& R_{[[N \sqcup\{y, u\}, N \sqcup(\{x, z\})]]}-R_{[[N \sqcup\{z, u\}, N \sqcup(\{x, y\})]]}=b+d .
\end{aligned}
$$

Hence we have

$$
\begin{aligned}
& R_{[[N \sqcup\{y, u\}, N \sqcup(\{x, z\})]]}=(a+b+c+d) / 2, \\
& R_{[[N \sqcup\{x, u\}, N \sqcup(\{y, z\})]]}=(a+b-c-d) / 2, \\
& R_{[[N \sqcup\{z, u\}, N \sqcup(\{x, y\})]]}=(a-b+c-d) / 2 .
\end{aligned}
$$

There are well-defined elements $\eta_{a}, \eta_{b}, \eta_{c}, \eta_{d}$ of $V_{M}^{\prime}$ such that

$$
\begin{aligned}
& \eta_{a}(\{x, y\})=0, \eta_{a}(\{y, z\})=0, \eta_{b}(\{x, y\})=0, \eta_{b}(\{y, z\})=1, \\
& \eta_{c}(\{x, y\})=1, \eta_{c}(\{y, z\})=0, \eta_{d}(\{x, y\})=1, \eta_{d}(\{y, z\})=1 .
\end{aligned}
$$

The assignment $\eta_{a} \mapsto a, \eta_{b} \mapsto b, \eta_{c} \mapsto c, \eta_{d} \mapsto d$ is a bijection $V_{M}^{\prime} \leftrightarrow \underline{D}_{M, N}^{u n}$ which establishes 46.14(c) in our case.

46.20. We now assume that $|M| \geq 4$ and that $(M, N)$ has the following property: there exists $k \in[0, \max (M \cup N)]$ such that $k \notin M \cup N$. We set

$$
h=n-|\{x>k ; x \in M\}|-2|\{x>k ; x \in N\}| .
$$

Clearly, $h<n$. Let

$$
\begin{aligned}
M^{\prime} & =\{x<k ; x \in M\} \sqcup\{x \geq k ; x+1 \in M\}, \\
N^{\prime} & =\{x<k ; x \in N\} \sqcup\{x \geq k ; x+1 \in N\} .
\end{aligned}
$$

Note that $M^{\prime}, N^{\prime}$ are disjoint subsets of $\mathbf{N}$ such that $\left|M^{\prime}\right|=|M|,\left|N^{\prime}\right|=|N|$ and

$$
\sum_{x \in M^{\prime}} x+2 \sum_{x \in N^{\prime}} x=\sum_{x \in M} x+2 \sum_{x \in N} x-(n-h)=h+m^{2}-m .
$$

In particular, $h \geq 0$. If $h \leq 1$, we see that $\left|M^{\prime}\right|=2 h$, hence $|M|=2 h<4$, a contradiction. Thus we have $h \in[2, n-1]$. We see also that $\left(M^{\prime}, N^{\prime}\right) \in X_{h}$. We define a bijection $M^{\prime} \stackrel{\sim}{\longrightarrow} M$ by $x \mapsto x$ if $x<k$ and $x \mapsto x+1$ if $x \geq k$. This induces 
a bijection $V_{M^{\prime}} \stackrel{\sim}{\longrightarrow} V_{M}$, hence a bijection $V_{M}^{\prime} \stackrel{\sim}{\longrightarrow} V_{M^{\prime}}^{\prime}$. Consider the two-sided cell $\mathbf{c}^{\prime}=\mathbf{c}_{M^{\prime}, N^{\prime}} \times \mathbf{c}_{0}$ of $\mathbf{W}_{I^{h}}$ (see 46.16) where $\mathbf{c}_{0}$ is the two-sided cell associated to the sign representation $\operatorname{sgn}_{h}$ of $\mathfrak{S}_{n-h}$. We have $\mathbf{c}^{\prime} \subset \mathbf{c}$ where $\mathbf{c}=\mathbf{c}_{M, N}$. Moreover, $\mathbf{c}^{\prime}, \mathbf{c}$ satisfy the assumptions (i),(ii) of 44.21. Consider the composite bijection

$$
V_{M}^{\prime} \stackrel{\sim}{\longrightarrow} V_{M^{\prime}}^{\prime} \stackrel{\sim}{\longrightarrow}\left(\underline{\bar{D}}^{h}\right)_{M^{\prime}, N^{\prime}}^{u n} \stackrel{\sim}{\longrightarrow}\left(\underline{\hat{D}}^{h}\right)_{\mathbf{c}^{\prime}}^{u n} \stackrel{\sim}{\longrightarrow} \underline{\hat{D}}_{M, N}^{u n} ;
$$

here the first bijection is as above; the second bijection comes from the induction hypothesis; the third bijection is $A^{\prime} \mapsto A \otimes A^{\prime}$ where $A=R_{\mathrm{sgn}_{n-h}} \in \widehat{P G L}_{n-h}^{u n}$; the fourth bijection comes from $44.21(\mathrm{~h})$. Using $44.21(\mathrm{~h})$ we see that this composite bijection has the required properties. This proves $46.14(\mathrm{c})$ in our case.

46.21. We now assume that $|M| \geq 4$ and that there exists $y>0$ such that $y \in$ $N, y-1 \notin N$. Recall that $M \cup N \subset[0, m+n-1]$. We can assume that $m=n$ so that $M \cup N \subset[0, t]$ where $t=2 n-1$. Let

$$
M^{\prime}=\{x ; t-x \in M\} \subset \mathbf{N}, N^{\prime}=\{x \in[0, t] ; t-x \notin M \cup N\} \subset \mathbf{N} .
$$

We have $M^{\prime} \cap N^{\prime}=\emptyset,\left|M^{\prime}\right|+2\left|N^{\prime}\right|=|M|+2(t+1)-2|M \cup N|=2 n$,

$$
\begin{aligned}
& \sum_{x ; t-x \in M} x+2 \sum_{x \in[0, t] ; t-x \notin M \cup N} x=\sum_{x \in M}(t-x)+2 \sum_{x \in[0, t]} x-2 \sum_{x \in M \cup N}(t-x) \\
= & |M| t-\sum_{x \in M} x+t^{2}+t-2|M| t-2|N| t+2 \sum_{x \in M} x+2 \sum_{x \in N} x \\
= & t^{2}+t-|M| t-2|N| t+\sum_{x \in M} x+2 \sum_{x \in N} x=n^{2} .
\end{aligned}
$$

We see that $\left(M^{\prime}, N^{\prime}\right) \in X_{n}^{n}$. We have a bijection $M^{\prime} \stackrel{\sim}{\longrightarrow} M, x \mapsto t-x$. This induces a bijection $V_{M^{\prime}} \stackrel{\sim}{\longrightarrow} V_{M}$ and a bijection $V_{M}^{\prime} \stackrel{\sim}{\longrightarrow} V_{M^{\prime}}^{\prime}$. Since $y \in N$, we have $y \notin M$, hence $t-y \notin M^{\prime}$. Since $y \in N$, we have $t-y \notin N^{\prime}$. Thus, $t-y \notin M^{\prime} \cup N^{\prime}$. If $y-1 \in M$, then $t-y+1 \in M^{\prime}$ and $t-y<t-y+1$. If $y-1 \notin M$, then $y-1 \notin M \cup N$ (since $y-1 \notin N$ ), hence $t-y+1 \in N^{\prime}$ and $t-y<t-y+1$. In any case we have $t-y+1 \in M^{\prime} \cup N^{\prime}$ and $t-y \in\left[0, \max \left(M^{\prime} \cup N^{\prime}\right)\right]$. By 46.20, 46.14(c) holds when $(M, N)$ is replaced by $\left(M^{\prime}, N^{\prime}\right)$. Consider the composite bijection

$$
V_{M}^{\prime} \stackrel{\sim}{\longrightarrow} V_{M^{\prime}}^{\prime} \stackrel{\sim}{\longrightarrow} \underline{\hat{D}}_{M^{\prime}, N^{\prime}}^{u n} \stackrel{\sim}{\longrightarrow} \underline{\hat{D}}_{M, N}^{u n}
$$

here the first bijection is as above; the second bijection is as in $46.14(\mathrm{c})$ for $\left(M^{\prime}, N^{\prime}\right)$; the third bijection is $A \mapsto A^{\circ}$; see 44.19(a). (Note that for $A \in \hat{D}^{u n}$ we have $A^{\circ}=\mathbf{d}(A)$ since $\mathbf{e}^{A}=1$ by 46.15 .) The composite bijection above is denoted by $\eta \mapsto A_{\eta}$. We have $A_{\eta}=\mathbf{d}\left(A_{\eta^{\prime}}\right)$ where $\eta \in V_{M}^{\prime}$ corresponds to $\eta^{\prime} \in V_{M^{\prime}}^{\prime}$ and $A_{\eta^{\prime}}$ is attached to $\eta^{\prime}$ by 46.14 (c) for $\left(M^{\prime}, N^{\prime}\right)$. For any $J \subset M$, let $J^{\prime} \subset M^{\prime}$ be the image of $J$ under $x \mapsto t-x$. Let $H \in \mathcal{V}_{M}$. Using 44.8(c) and [L15, (1.4.1)] we have

$$
\left(A_{\eta}: R_{[[N \sqcup H, N \sqcup(M-H)]]}\right)=\left(\mathbf{d}\left(A_{\eta^{\prime}}\right): \mathbf{d}\left(R_{\left[\left[N^{\prime} \sqcup\left(M^{\prime}-H^{\prime}\right), N^{\prime} \sqcup H^{\prime}\right]\right]}\right) .\right.
$$

(We have $[[N \sqcup H, N \sqcup(M-H)]] \otimes \operatorname{sgn}=\left[\left[N^{\prime} \sqcup\left(M^{\prime}-H^{\prime}\right), N^{\prime} \sqcup H^{\prime}\right]\right]$.) This equals

$$
\left(A_{\eta^{\prime}}: R_{\left[\left[N^{\prime} \sqcup\left(M^{\prime}-H^{\prime}\right), N^{\prime} \sqcup H^{\prime}\right]\right]}\right)=2^{-\left|M^{\prime}\right| / 2+1}(-1)^{\eta^{\prime}\left(\left(M^{\prime}-H^{\prime}\right) * t_{M^{\prime}}^{-1}(1)\right)} .
$$

(We have used 46.14(c) for $\left(M^{\prime}, N^{\prime}\right)$.) By definition we have

$$
\begin{aligned}
\eta^{\prime}\left(\left(M^{\prime}-H^{\prime}\right) * t_{M^{\prime}}^{-1}(1)\right) & =\eta^{\prime}\left((M-H)^{\prime} * t_{M}^{-1}(0)^{\prime}\right)=\eta^{\prime}\left(\left((M-H) * t_{M}^{-1}(0)\right)^{\prime}\right) \\
& =\eta\left((M-H) * t_{M}^{-1}(0)\right)=\eta\left(H * t_{M}^{-1}(1)\right)
\end{aligned}
$$


so that 46.14(c) holds in our case. For the last equality we note that

$$
\begin{aligned}
& \eta\left((M-H) * t_{M}^{-1}(0)\right)+\eta\left(H * t_{M}^{-1}(1)\right)=\eta\left((M-H) * t_{M}^{-1}(0) * H * t_{M}^{-1}(1)\right) \\
& \quad=\eta(M * M)=\eta(\emptyset)=0 .
\end{aligned}
$$

46.22. We now assume that $(M, N) \in X_{n}^{m}$ does not satisfy the assumptions of $46.18,46.19,46.20$ or 46.21 . Then $|M| \geq 6$ and there exist $r \geq 0, s \geq 3$ such that

$$
N=\{0,1, \ldots, r-1\}, M=\{r, r+1, r+2, \ldots, r+2 s-1\} .
$$

Note that $(M, N)$ has the same image in $\bar{X}_{n}$ as $\left(M^{\prime}, N^{\prime}\right)=(\{0,1,2, \ldots, 2 s-1\}, \emptyset)$. Since the statements of $46.14(\mathrm{c})$ for $(M, N)$ and $\left(M^{\prime}, N^{\prime}\right)$ are equivalent, it is enough to prove $46.14(\mathrm{c})$ for $\left(M^{\prime}, N^{\prime}\right)$ instead of $(M, N)$. Thus we may assume that $(M, N)=(\{0,1,2, \ldots, 2 s-1\}, \emptyset)$ with $s \geq 3$. We have $(M, N) \in X_{s^{2}}^{s}$.

If $\Phi$ is an admissible arrangement of $M$ let $\mathcal{C}_{\Phi}$ be the set of all subsets $E$ of $M$ with the following property: if $(x, y)$ is a pair in $\Phi$, then $x \in E$ if and only if $y \in E$. Note that $\mathcal{C}_{\Phi}$ is a subspace of the vector space $V_{M}$ of dimension $s$ and containing $M$. Clearly, $\Psi \mapsto\left(\Psi^{0} \cup(\Phi-\Psi)^{1}\right)^{\sharp}$ is a bijection between the sets of subsets of $\Phi$ and $\mathcal{C}_{\Phi}$. Via this bijection the function $\Psi \mapsto|\hat{\Phi} \cap \Psi| \bmod 2$ (for $\hat{\Phi} \subset \Phi$ that $|\hat{\Phi}|$ is odd) can be viewed as a linear function $\mathcal{C}_{\Phi} \rightarrow \mathbf{F}_{2}$. This gives a bijection between $\{\hat{\Phi} ; \hat{\Phi} \subset \Phi,|\hat{\Phi}|=$ odd $\}$ and the set of linear functions $\mathcal{C}_{\Phi} \rightarrow \mathbf{F}_{2}$ which take the value 1 on $M$. Using the notation $\langle E\rangle$ instead of $[[S, T]]$ where $(S, T) \in \zeta^{-1}(M, N)$ and $E=S^{\sharp} \in \tilde{\mathcal{V}}_{M}$ we see that the elements $c(M, N, \Phi, \hat{\Phi})$ (see 46.13) are the same as the elements

$$
c(M, N, \Phi ; \xi)=\frac{1}{2} \sum_{E \in \mathcal{C}_{\Phi}}(-1)^{\xi(E)} \phi_{\langle E\rangle} \in \mathcal{R}(\tilde{\mathbf{W}})
$$

for various linear functions $\xi: \mathcal{C}_{\Phi} \rightarrow \mathbf{F}_{2}$ such that $\xi(M)=1$.

Now let $\Phi^{\prime}$ be another admissible arrangement of $M$ and let $\xi^{\prime}: \mathcal{C}_{\Phi^{\prime}} \rightarrow \mathbf{F}_{2}$ be a linear form such that $\xi^{\prime}(M)=1$. We have

$$
\begin{aligned}
& \left(R_{c(M, N, \Phi ; \xi)}: R_{c\left(M, N, \Phi^{\prime} ; \xi^{\prime}\right)}\right)=\frac{1}{4} \sum_{E \in \mathcal{C}_{\Phi}, E^{\prime} \in \mathcal{C}_{\Phi^{\prime}}}(-1)^{\xi(E)+\xi^{\prime}\left(E^{\prime}\right)}\left(R_{\langle E\rangle}: R_{\left\langle E^{\prime}\right\rangle}\right) \\
& =\frac{1}{4} \sum_{E \in \mathcal{C}_{\Phi} \cap \mathcal{C}_{\Phi^{\prime}}}(-1)^{\xi(E)+\xi^{\prime}(E)}-\frac{1}{4} \sum_{E \in \mathcal{C}_{\Phi} \cap \mathcal{C}_{\Phi^{\prime}}}(-1)^{\xi(E)+\xi^{\prime}(M-E)} \\
& =\frac{1}{2} \sum_{E \in \mathcal{C}_{\Phi} \cap \mathcal{C}_{\Phi^{\prime}}}(-1)^{\xi(E)+\xi^{\prime}(E)}=\left|\left\{\eta \in \operatorname{Hom}\left(V_{M}, \mathbf{F}_{2}\right) ;\left.\eta\right|_{\mathcal{C}_{\Phi}}=\xi,\left.\eta\right|_{\mathcal{C}_{\Phi^{\prime}}}=\xi^{\prime}\right\}\right| .
\end{aligned}
$$

Now let $k \in[0,2 s-2]$ and let $M^{\prime}=\{0,1,2, \ldots, k-1, k+1, \ldots, 2 s-2\}, N^{\prime}=\{k\}$. We have $\sum_{x \in M^{\prime}} x+\sum_{x \in N^{\prime}} x=h+s^{2}-s$ where $h=s^{2}-(2 s-k-1)$. Since $s \geq 3$ and $k \in[0,2 s-2]$, we have $h \in\left[4, s^{2}-1\right]$ and $\left(M^{\prime}, N^{\prime}\right) \in \bar{X}_{h}^{s}$.

Consider the two-sided cell $\mathbf{c}^{\prime}=\mathbf{c}_{M^{\prime}, N^{\prime}} \times \mathbf{c}_{0}$ of $\mathbf{W}_{I^{h}}$ (see 46.16) where $\mathbf{c}_{0}$ is the two-sided cell associated to the sign representation $\operatorname{sgn}_{h}$ of $\mathfrak{S}_{n-h}$. We have $\mathbf{c}^{\prime} \subset \mathbf{c}$ where $\mathbf{c}=\mathbf{c}_{M, N}$.

Define an imbedding $j: M^{\prime} \rightarrow M$ by $j(x)=x$ if $x \in[0, k-1], j(x)=x+1$ if $x \in[k+1,2 s-2]$. Let $V_{M}^{0}=\left\{E \in V_{M} ;|E \cap\{k, k+1\}|=\right.$ even $\}$, a hyperplane in $V_{M}$. If $E \in V_{M}^{0}$, then $j^{-1}(E) \in V_{M^{\prime}}$.

Let $\eta_{1}, \eta_{2}$ be two elements of $V_{M}^{\prime}$ such that

(a) $\eta_{1}(E)+\eta_{2}(E)=|E \cap\{k, k+1\}| \bmod 2$ for all $E \in V_{M}$ and $\eta_{1}(\{k, k+1\})=$ $\eta_{2}(\{k, k+1\})=0$. 
We define a linear function $\eta^{\prime}: V_{M^{\prime}} \rightarrow \mathbf{F}_{2}$ by $\eta^{\prime}\left(E^{\prime}\right)=\eta_{1}\left(j\left(E^{\prime}\right)\right)=\eta_{2}\left(j\left(E^{\prime}\right)\right)$ for $E^{\prime} \in V_{M^{\prime}}$. (The last equality follows from (a) and the fact that $j\left(E^{\prime}\right) \cap\{k, k+1\}=$ $\emptyset$.) We have $\eta^{\prime}\left(M^{\prime}\right)=1$. (We use that

$$
1=\eta_{1}(M)=\eta_{1}\left(j\left(M^{\prime}\right) *\{k, k+1\}\right)=\eta_{1}\left(j\left(M^{\prime}\right)\right)
$$

which follows from (a).) Thus we have $\eta^{\prime} \in V_{M^{\prime}}^{\prime}$. Let $A_{\eta^{\prime}}$ be the object of $\left(\hat{\bar{D}}^{h}\right)_{M^{\prime}, N^{\prime}}^{u n}$ associated to $\eta^{\prime}$ by the induction hypothesis applied to $\left(M^{\prime}, N^{\prime}\right)$. Then $R_{\operatorname{sgn}_{h}} \otimes A_{\eta^{\prime}} \in$ $\left(\hat{D}^{u n}\right)_{\mathbf{c}^{\prime}}$ is defined. We set $\alpha_{\eta_{1}, \eta_{2}}=\operatorname{tind}_{D^{h}}^{D}\left(R_{\mathrm{sgn}_{h}} \otimes A_{\eta^{\prime}}\right)$ (see 44.20). By definition, this is an element of $\mathcal{K}^{u n}(D)$ which is an $\mathbf{N}$-linear combination of objects in $D_{M, N}^{u n}$. Now let $(S, T) \in \zeta^{-1}(M, N)$. Using $44.20(\mathrm{~h})$ we see that $\left(\alpha_{\eta_{1}, \eta_{2}}: R_{[[S, T]]}\right)$ is 0 if $|S \cap\{k, k+1\}| \neq 1$, while if $|S \cap\{k, k+1\}|=1$, it is

(b) $\left(A_{\eta^{\prime}}: R_{\left[\left[S^{\prime}, T^{\prime}\right]\right]}\right)$

where $\left(S^{\prime}, T^{\prime}\right) \in \zeta^{-1}\left(M^{\prime}, N^{\prime}\right)$ is given by

$$
\begin{aligned}
& S^{\prime}=\{x<k ; x \in S\} \sqcup\{k\} \sqcup\{x>k ; x+1 \in S\}, \\
& T^{\prime}=\{x<k ; x \in T\} \sqcup\{k\} \sqcup\{x>k ; x+1 \in T\} .
\end{aligned}
$$

By the induction hypothesis, the expression (b) is equal to

$$
2^{-\left|M^{\prime}\right| / 2+1}(-1)^{\eta^{\prime}\left(t_{M^{\prime}}^{-1}(1) *\left(S^{\prime}-\{k\}\right)\right)}=2^{-s+2}(-1)^{\eta_{1}\left(S^{\sharp}\right)}=2^{-s+2}(-1)^{\eta_{2}\left(S^{\sharp}\right)} .
$$

Hence, if $\Phi$ is an admissible arrangement of $M$ and $\xi: \mathcal{C}_{\Phi} \rightarrow \mathbf{F}_{2}$ is a linear function such that $\xi(M)=1$, then

$$
\begin{aligned}
& \left(\alpha_{\eta_{1}, \eta_{2}}: R_{c(M, N, \Phi ; \xi)}\right)=\frac{1}{2} \sum_{E \in \mathcal{C}_{\Phi}}(-1)^{\xi(E)}\left(\alpha_{\eta_{1}, \eta_{2}}: R_{\langle E\rangle}\right) \\
& =\frac{1}{2} \sum_{\substack{E \in \mathcal{C}_{\Phi} ; \\
|E \cap\{k, k+1\}|=\text { even }}}(-1)^{\xi(E)} 2^{-s+2}(-1)^{\eta_{1}(E)} \\
& =\sum_{\substack{E \in \mathcal{C}_{\Phi} ; \\
|E \cap\{k, k+1\}|=\text { even }}} 2^{-s+1}(-1)^{\eta_{1}(E)+\xi(E)} .
\end{aligned}
$$

This is equal to the number of elements in $\left\{\eta_{1}, \eta_{2}\right\}$ whose restriction to $\mathcal{C}_{\Phi}$ is equal to $\xi$. (It is 2,1 or 0 .) We now apply [L14, 9.2] to $Y=V_{M}$ with its basis $\{\{0,1\},\{1,2\}, \ldots,\{2 s-2,2 s-1\}\}$ and to the family of elements $R_{c(M, N, \Phi, \xi)}$ for various $\Phi, \xi$ as above and the family of elements $\alpha_{\eta_{1}, \eta_{2}}$ for various $\eta_{1}, \eta_{2}, k$ as above. (These elements are $\mathbf{N}$-linear combinations of objects in $\hat{D}_{M, N}^{u n}$ ) We see that there exists a bijection $V_{M}^{\prime} \leftrightarrow \hat{D}_{M, N}^{u n}, \eta \leftrightarrow A_{\eta}$ such that for any $\eta \in V_{M}^{\prime}$ we have $R_{c(M, N, \Phi, \xi)}=\sum_{\eta \in V_{M}^{\prime} ; \eta \mid \mathcal{C}_{\Phi}=\xi} A_{\eta}$ for any $\Phi, \xi$ as above and $\alpha_{\eta_{1}, \eta_{2}}=A_{\eta_{1}}+A_{\eta_{2}}$ for any $\eta_{1}, \eta_{2}, k$ as above.

Now let $E \in \tilde{\mathcal{V}}_{M}$. We can rephrase 46.13(b) as follows: there exists an admissible arrangement $\Phi$ of $M$ such that $E \in \mathcal{C}_{\Phi}$; moreover,

$$
\phi_{\langle E\rangle}=2^{-s+1} \sum_{\xi \in \operatorname{Hom}\left(\mathcal{C}_{\Phi}, \mathbf{F}_{2}\right) ; \xi(M)=1}(-1)^{\xi(E)} c(M, N, \Phi ; \xi) .
$$

For $\eta \in V_{M}^{\prime}$ we then have

$$
\left(A_{\eta}: R_{\langle E\rangle}\right)=2^{-s+1} \sum_{\substack{\xi \in \operatorname{Hom}\left(\mathcal{C}_{\Phi}, \mathbf{F}_{2}\right) ; \\ \xi(M)=1}}(-1)^{\xi(E)}\left(A_{\eta}: R_{c(M, N, \Phi ; \xi)}\right)=2^{-s+1}(-1)^{\eta(E)} .
$$


We see that 46.14(c) holds in our case. This completes the proof of 46.14(c).

46.23. In this subsection we assume that $p=2$. Let $P$ be a proper parabolic subgroup of $G^{0}$ such that $N_{D} P \neq \emptyset$ and such that (setting $G^{\prime}=N_{G} P / U_{P}, D^{\prime}=$ $\left.N_{D} P / U_{P}\right)$ we have $\hat{D}^{\prime \text { unc }} \neq \emptyset$. Let $\bar{D}^{\prime}, \bar{G}^{\prime}$ be the quotient of $D^{\prime}, G^{\prime}$ by the translation action of $\mathcal{Z}_{G^{\prime}}^{0}$. Let $\pi: D^{\prime} \rightarrow \bar{D}^{\prime}$ be the obvious map. From the induction hypothesis we see that $P / U_{P}$ is of type $D_{r}$ (with $r$ an odd square $\geq 9$ ) or a torus, that $\hat{D}^{\prime u n c}$ has exactly one object $A$ up to isomorphism and that $\operatorname{supp}(A)$ is contained in the inverse image under $\pi$ of the variety of unipotent elements of $\bar{G}^{\prime}$ contained in $\bar{D}^{\prime}$. Let $\hat{D}^{u n, P}$ be the subcategory of $\hat{D}^{u n}$ consisting of objects which are isomorphic to direct summands of $\operatorname{ind}_{D^{\prime}}^{D}(A)$. From 27.2 and 11.9 we see that the set of isomorphism classes in $\hat{D}^{u n, P}$ is in bijection with the set of isomorphism classes of simple modules of $\mathbf{Q}\left[W_{n-r}\right]$. Since any noncuspidal object of $\hat{D}^{u n}$ belongs to $\hat{D}^{u n, P}$ for a $P$ as above (unique up to $G^{0}$-conjugacy), we see that the number of noncuspidal objects of $\underline{D}^{u n}$ is equal to

$$
\sum_{k>0, s \geq 0, s \text { odd, } s^{2}+k=n} p_{2}(k)
$$

where $p_{2}(k)$ is the number of irreducible representations of $W_{k}$ up to isomorphism. Now let $x_{n}=\left|\underline{\hat{D}}^{u n}\right|$. From $46.14(\mathrm{c})$ we see that $x_{n}=\left|X_{n}\right|$. Since $\left|X_{n}\right|$ is known from [L7] we see that

$$
x_{n}=\left|X_{n}\right|=\sum_{k \geq 0, s \geq 0, s \text { odd, } s^{2}+k=n} p_{2}(k)
$$

where $p_{2}(0)=1$. Comparing with (a) we see that the number of cuspidal objects of $\underline{D}^{u n}$ is 1 if $n=s^{2}$ for some odd $s \geq 3$ and is 0 otherwise. From 12.9 we see that the set of irreducible cuspidal admissible complexes on $D$ (up to isomorphism) is empty unless $n=s^{2}$ for some odd $s \geq 3$ in which case it has exactly one object (whose support is necessarily contained in the unipotent variety). Since any object of $\underline{\hat{D}}^{u n}$ is an admissible complex on $D$ we see that 46.14(d) holds for $D$.

This completes the inductive proof of the statements 46.14(a)-(d).

46.24. Let $(M, N)=(\{0,1,2, \ldots, 2 s-1\}, \emptyset) \in X_{n}^{s}, n=s^{2}$ with $s$ odd, $s \geq 3$. Define a linear function $\eta: V_{M} \rightarrow \mathbf{F}_{2}$ by

$$
\eta(E)=\left|E \cap t_{M}^{-1}(0)\right| \bmod 2=\left|E \cap t_{M}^{-1}(1)\right| \bmod 2 .
$$

Since $s$ is odd we have $\eta(M)=1$, hence $\eta \in V_{M}^{\prime}$. In the setup of 46.22 we show:

(a) $A_{\eta} \in \hat{D}^{u n c}$.

For $w \in \mathbf{W}$, we have (in view of 46.22 and $44.7(\mathrm{i})$ ):

$$
\begin{aligned}
& \left(A_{\eta}: g r_{1}\left(K_{D}^{w}\right)\right)=(-1)^{\operatorname{dim} G} \frac{1}{2} \sum_{E \in \tilde{\mathcal{V}}_{M}} \operatorname{tr}(w \varpi,\langle E\rangle)\left(A_{\eta}: R_{\langle E\rangle}\right) \\
& =(-1)^{\operatorname{dim} G} \frac{1}{2} \sum_{E \in \tilde{\mathcal{V}}_{M}} \operatorname{tr}(w \varpi,\langle E\rangle) 2^{-s+1}(-1)^{\eta(E)} .
\end{aligned}
$$

By 44.14(a), the condition that $A_{\eta}$ is cuspidal is that $\left(A_{\eta}: g r_{1}\left(K_{D}^{w}\right)\right)=0$ whenever $w \in \mathbf{W}$ is not $D$-anisotropic. Thus it is enough to show that

$$
\sum_{E \in \tilde{\mathcal{V}}_{M}} \operatorname{tr}(w \varpi,\langle E\rangle)(-1)^{\left|E \cap t_{M}^{-1}(0)\right|}=0
$$


whenever $w \in \mathbf{W}=W_{n}^{\prime}$ satisfies the condition: $w$ is not $D$-anisotropic or equivalently, the condition: $w s_{n} \in W_{n}$ has no eigenvalue 1 in the reflection representation of $W_{n}$. Note that (b) holds by [L3, (22.5.2) V]. (In that reference the words: "elements of $W^{\prime \prime}$ " should be replaced by: "elements of $W^{\prime}-W^{\prime}$.)

Theorem 46.25. Assume that $p$ satisfies the following condition: if $G^{0}$ has a factor of type $E_{8}$ or $F_{4}$, then $p \neq 2$. Then:

(a) If $A$ is a unipotent cuspidal character sheaf on $D$, then $A$ is clean (see 44.7).

(b) If $A$ is a unipotent character sheaf on $D$, then for any $w \in \mathbf{W}, i \in \mathbf{Z}$ such that $\left(A: H^{i}\left(\bar{K}_{D}^{w}\right)\right) \neq 0$ we have $i=\operatorname{dim} \operatorname{supp}(A) \bmod 2$ (or equivalently $\left.\mathbf{e}^{A}=(-1)^{\operatorname{codim}(\operatorname{supp}(A))}\right)$.

By the results in $\S 45$ we are reduced to the case where $G^{0}$ is simple and $\mathcal{Z}_{G}=\{1\}$. If $D=G^{0}$, (a) is a special case of 46.1(b); the fact that (a) implies (b) is proved in this case as in [L3, IV, V]. If $D \neq G^{0}$, then (a) and (b) follow from 46.4(a),(b); 46.7(a),(c); 46.8(a),(d); 46.14(a),(b). This completes the proof.

46.26. Let $e$ be a pinning (see 1.6) of $G^{0}$ which projects to $\left(B^{*}, T\right)$ (see 28.5) under the map $p$ in 1.6. We can find $d \in D$ such that $\beta:=\operatorname{Ad}(d): G^{0} \rightarrow G^{0}$ preserves $e$. Moreover, $\beta$ depends only on $D$ (not on $d$ ). Note that $\beta$ has finite order, say $r$.

Let $\mathbb{G}$ be a connected reductive algebraic group over $\mathbf{C}$ with a fixed Borel subgroup $\mathbb{B}$, a fixed maximal torus $\mathbb{T} \subset \mathbb{B}$ and a fixed pinning $\underline{e}$ which projects to $(\mathbb{B}, \mathbb{T})$ such that $\mathbb{G}$ is a Langlands dual of $G^{0}$. In particular, $T, \mathbb{T}$ are Langlands dual tori. There is a unique automorphism $\gamma: \mathbb{G} \rightarrow \mathbb{G}$ preserving $\underline{e}$ such that the restriction of $\gamma$ to $\mathbb{T}$ corresponds to (is "contragredient of") the restriction of $\beta$ to $T$ under the Langlands duality between $T$ and $\mathbb{T}$. Note that $\gamma$ has order $r$.

A $\mathbb{G}$-conjugacy class $C$ in $\mathbb{G}$ is said to be special if some/any $g \in C$ is such that $g_{s}$ has finite order not divisible by $p, g_{u}$ is a special unipotent element of the connected reductive group $Z_{\mathbb{G}}\left(g_{s}\right)^{0}$ (see [L14, (13.1.1)]).

Let $C$ be a special $\mathbb{G}$-conjugacy class in $\mathbb{G}$ which is $\gamma$-stable. For $g \in C$ let $A\left(g_{u}\right)$ be the group of components of the centralizer of $g_{u}$ in $Z_{\mathbb{G}}\left(g_{s}\right)^{0}$, let $\bar{A}\left(g_{u}\right)$ be the canonical quotient of $A\left(g_{u}\right)$ defined in [L14, p. 343] (in terms of $g_{u}, Z_{\mathbb{G}}\left(g_{s}\right)^{0}$ instead of $\left.u, G_{1}\right)$ and let $I\left(g_{u}\right)$ be the kernel of the canonical homomorphism $A\left(g_{u}\right) \rightarrow$ $\bar{A}\left(g_{u}\right)$. Let $\tilde{\mathbb{A}}(g)=\left\{(a, j) \in \mathbb{G} \times \mathbf{Z} / r \mathbf{Z} ; a \gamma^{j}(g) a^{-1}=g\right\} / Z_{\mathbb{G}}(g)^{0}$ be a group with multiplication $(a, j)\left(a^{\prime}, j^{\prime}\right)=\left(a \gamma^{j}\left(a^{\prime}\right), j+j^{\prime}\right)$. We identify $Z_{\mathbb{G}}(g)^{0}$ with a (normal) subgroup of $\widetilde{\mathbb{A}}(g)$ by $a \mapsto(a, 0)$ and we set $\mathbb{A}(g)=\tilde{\mathbb{A}}(g) / Z_{\mathbb{G}}(g)^{0}$ (a finite group). Let $\mathbb{A}(g) \rightarrow \mathbf{Z} / r \mathbf{Z}$ be the (surjective) homomorphism induced by $(a, j) \mapsto j$. Since $Z_{Z_{\mathbb{G}}\left(g_{s}\right)^{0}}\left(g_{u}\right)^{0}=Z_{\mathbb{G}}(g)^{0}$ we see that $I\left(g_{u}\right)$ is naturally a subgroup of $\mathbb{A}(g)$. From the definitions we see that that in fact $I\left(g_{u}\right)$ is normal in $\mathbb{A}(g)$. Let $\mathcal{G}_{g}=\mathbb{A}(g) / I\left(g_{u}\right)$. The homomorphism $\mathbb{A}(g) \rightarrow \mathbf{Z} / r \mathbf{Z}$ induces a surjective a homomorphism $\mathcal{G}_{g} \rightarrow$ $\mathbf{Z} / r \mathbf{Z}$. For $j \in \mathbf{Z} / r \mathbf{Z}$ let $\mathcal{G}_{g}^{j}$ be the inverse image of $j$ under this homomorphism. Let $\mathcal{G}_{C}=\bigsqcup_{g \in C} \mathcal{G}_{g}$. Now $\mathbb{G}$ acts on $\mathcal{G}_{C}$ : if $x \in \mathbb{G}, g \in C$, then $\operatorname{Ad}(x)$ induces an isomorphism $\mathcal{G}_{g} \stackrel{\sim}{\longrightarrow} \mathcal{G}_{x g x^{-1}}$. Let $\mathcal{G}_{C}^{1}=\bigsqcup_{g \in C} \mathcal{G}_{g}^{1}$, a $\mathbb{G}$-stable subset of $\mathcal{G}_{C}$. For any $g \in C$, the set of $\mathbb{G}$-orbits on $\mathcal{G}_{C}^{1}$ is in natural bijection with the (finite) set of $\mathcal{G}_{g}$-conjugacy classes in $\mathcal{G}_{g}^{1}$. Thus $\mathbb{G}$ acts on $\mathcal{G}_{C}^{1}$ with finitely many orbits. This makes $\mathcal{G}_{C}^{1}$ into an algebraic variety (a finite union of homogeneous spaces for $\mathbb{G}$ ).

Let $\mathfrak{P}_{\gamma}$ be the set of all triples $(C, X, \mathcal{E})$ where $C$ is a $\gamma$-stable special $\mathbb{G}$-conjugacy class in $\mathbb{G}, X$ is a $\mathbb{G}$-orbit in $\mathcal{G}_{C}^{1}$ and $\mathcal{E}$ is an irreducible $\mathbb{G}$-equivariant local system on $X$ (up to isomorphism). Let $\mathfrak{P}_{\gamma}^{u n}$ be the set of all $(C, X, \mathcal{E}) \in \mathfrak{P}_{\gamma}$ such that $C$ is a unipotent $\mathbb{G}$-conjugacy class in $\mathbb{G}$. 
46.27. We have $\mathfrak{P}_{\gamma}^{\text {un }}=\bigsqcup_{C} \mathfrak{P}_{\gamma, C}^{\text {un }}$ where $C$ runs over the set of $\gamma$-stable special unipotent classes in $\mathbb{G}$ and $\mathfrak{P}_{\gamma, C}^{u n}$ is the set of triples in $\mathfrak{P}_{\gamma}^{u n}$ whose first component is $C$. Under the Springer correspondence, the set of $\gamma$-stable special unipotent classes in $\mathbb{G}$ is in bijection with the set of special irreducible representations $E_{0}$ (up to isomorphism) of the Weyl group of $\mathbb{G}$ or of $G^{0}$ whose character is fixed by $\epsilon: \mathbf{W} \rightarrow \mathbf{W}$ and hence in bijection (via $E_{0} \mapsto \mathbf{c}_{E_{0}}$, see 43.6 ) with the set of $\epsilon$-stable two-sided cells of $\mathbf{W}$; let $C_{\mathbf{c}}$ be the special unipotent class corresponding to the two-sided cell c. Assume that $p$ is as in 46.25. We have the following result:

(a) For any $\epsilon$-stable two-sided cell $\mathbf{c}$ in $\mathbf{W}$ there is a natural bijection $\hat{D}_{\mathbf{c}}^{u n} \leftrightarrow$ $\mathfrak{P}_{\gamma, C_{\mathbf{c}}}^{u n}$.

By the results in $\S 45$ we are reduced to the case where $G^{0}$ is simple and $\mathcal{Z}_{G}=\{1\}$. If $D=G^{0}$, (a) is established in [L3, IV, V]. If $D \neq G^{0}$, then (a) follows from 46.4(d), 46.7, 46.8, 46.14(c).

By taking disjoint union over the various c we obtain a bijection $\hat{D}^{u n} \leftrightarrow \mathfrak{P}_{\gamma}^{u n}$. We will show elsewhere that this extends to a natural bijection $\hat{D} \leftrightarrow \mathfrak{P}_{\gamma}$. (See [L3, IV, V] for the case where $G=G^{0}$.)

\section{REFERENCES}

[BBD] A. Beilinson, J. Bernstein and P. Deligne, Faisceaux pervers, Astérisque 100 (1982). MR751966 (86g:32015)

[DL] P. Deligne and G. Lusztig, Representations of reductive groups over finite fields, Ann. Math. 103 (1976), 103-161. MR0393266 (52:14076)

[KL1] D. Kazhdan and G. Lusztig, Representations of Coxeter groups and Hecke algebras, Inv. Math. 53 (1979), 165-184. MR560412 (81j:20066)

[KL2] D. Kazhdan and G. Lusztig, Schubert varieties and Poincaré duality, Proc. Symp. Pure Math. 36 (1980), 185-203. MR.573434 (84g:14054)

[L3] G. Lusztig, Character sheaves, I, Adv. Math. 56 (1985), 193-237; II, vol. 57, 1985, pp. 226265; III, vol. 57, 1985, pp. 266-315; IV, vol. 59, 1986, pp. 1-63; V, vol. 61, 1986, pp. 103-155. MR.792706 (87b:20055)

[L7] G. Lusztig, Irreducible representations of finite classical groups, Inv. Math. 43 (1977), 125-175. MR0463275 (57:3228)

[L9] G. Lusztig, Character sheaves on disconnected groups, I, Represent. Theory (electronic) 7 (2003), 374-403; II, vol. 8, 2004, pp. 72-124; III, vol. 8, 2004, pp. 125-144; IV, vol. 8, 2004, pp. 145-178; Errata, vol. 8, 2004, pp. 179-179; V, vol. 8, 2004, pp. 346-376; VI, vol. 8, 2004, pp. 377-413; VII, vol. 9, 2005, pp. 209-266; VIII, vol. 10, 2006, pp. 314-352; IX, vol. 10, 2006, pp. 353-379. MR2017063 (2006d:20090a) MR2077486 (2005h:20111) MR2084488

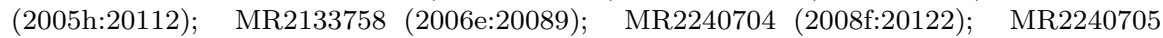
(2008e:20078)

[L12] G. Lusztig, Hecke algebras with unequal parameters, CRM Monograph Ser.18, Amer. Math. Soc., 2003. MR.1974442 (2004k:20011)

[L14] G. Lusztig, Characters of reductive groups over a finite field, Ann. Math. Studies 107, Princeton Univ. Press, 1984. MR.742472 (86j:20038)

[L15] G. Lusztig, Unipotent characters of the even orthogonal groups over a finite field, Trans. Amer. Math. Soc. 272 (1982), 733-751. MR662064 (83i:20035)

[Os] V. Ostrik, A remark on character sheaves, Adv. in Math. 192 (2005), 218-224. MR2122285 (2006j:20066)

[Sh] T. Shoji, Character sheaves and almost characters of reductive groups, II, Adv. in Math. 111 (1995), 314-354. MR1318530 (95k:20069)

[S] N. Spaltenstein, Classes unipotentes et sous-groupes de Borel, Lecture Notes in Math. 946, Springer-Verlag, New York, 1982. MR672610 (84a:14024)

Department of Mathematics, Massachusetts Institute of Technology, Cambridge, Massachusetts 02139 